\title{
TNFa-mediated subtype switch and tumor progression in pancreatic cancer
}

\author{
DISSERTATION \\ For the award of the degree \\ "Doctoral of Philosophy (Ph.D.)" \\ Division of Mathematics and Natural Science \\ of the Georg-August-Universität Göttingen \\ within the doctoral program "Molecular Medicine" \\ of the Georg-August University School of Science (GAUSS) \\ Submitted by \\ Mengyu Tu
}

Born in

Korla, China

Göttingen, July 2021 



\author{
Members of the Thesis Committee \\ Dr. Shiv K. Singh ( $\mathbf{1}^{\text {st }}$ Referee) \\ Department of Gastroenterology GI Oncology \& Endocrinology \\ University Medical Center, Göttingen
}

Prof. Dr. Holger Reichardt (2 ${ }^{\text {nd }}$ Referee)

Department of Cellular and Molecular Immunology

University Medical Center, Göttingen

Prof. Dr. Dieter Kube

Department of Haematology and Oncology

University Medical Center, Göttingen

\title{
Extended Examination Board Members:
}

Prof. Dr. Argyris Papantonis

Department of Pathology

University Medical Center, Göttingen

Dr. Ufuk Günesdogan

Department of Developmental Biology

University of Göttingen

Dr. Nico Posnien

Department of Developmental Biology

University of Göttingen

Date of submission of thesis: 15.07.2021

Date of the oral examination: 08.09.2021 



\section{AFFIDAVIT}

I hereby declare that the thesis entitled "TNFa mediated subtype switch and tumor progression in pancreatic cancer" has been written independently and with no other sources and aids than quoted.

Mengyu Tu

Göttingen. 15.07.2021 



\section{Table of Contents}

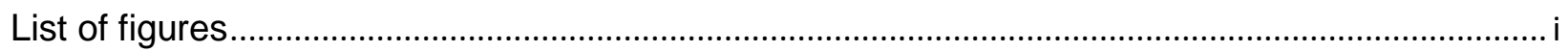

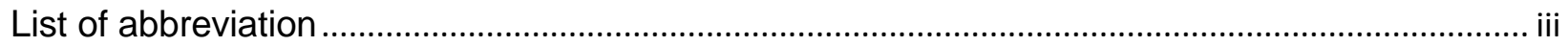

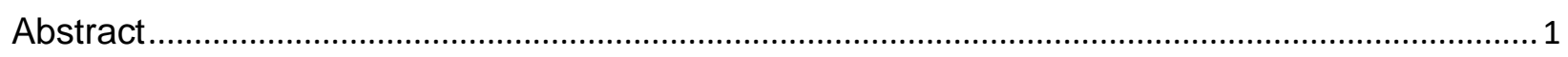

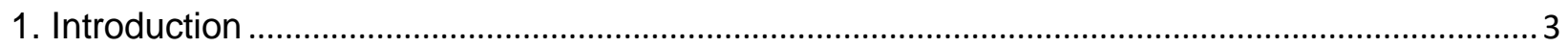

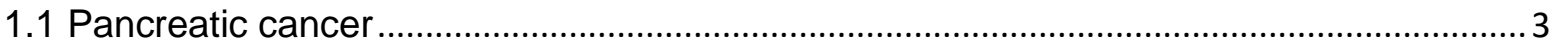

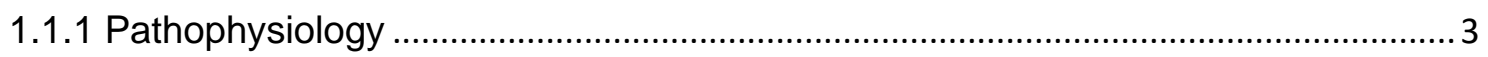

1.1.2 Tumor initiation and progression........................................................................ 4

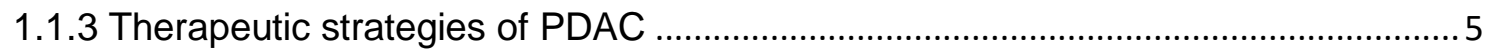

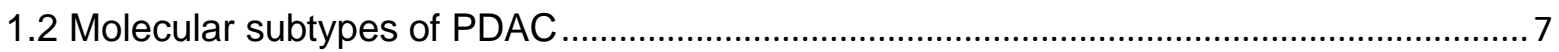

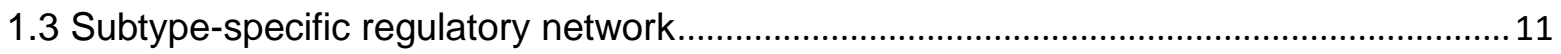

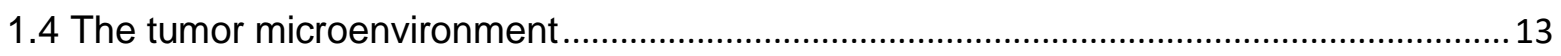

1.4.1 Inflammatory tumor stroma microenvironment .................................................... 14

1.4.1.1 Role of inflammation in tumor progression ................................................ 14

1.4.1.2 Tumor stroma microenvironment in disease progression............................17

1.4.2 Reciprocal interaction between stromal and tumors cells in PDAC prognosis .....18

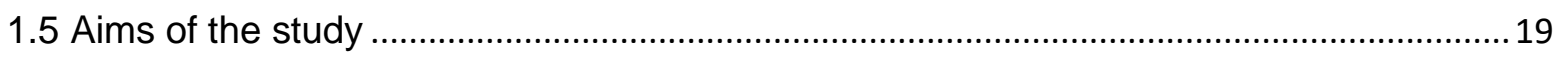

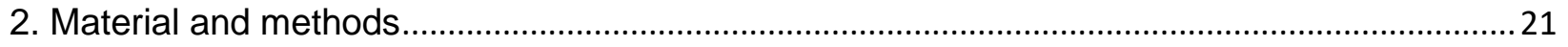

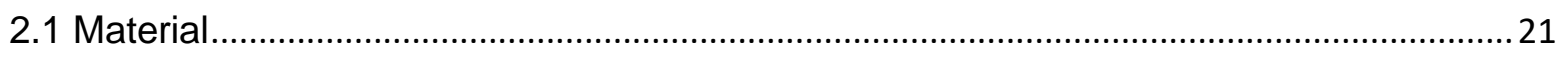

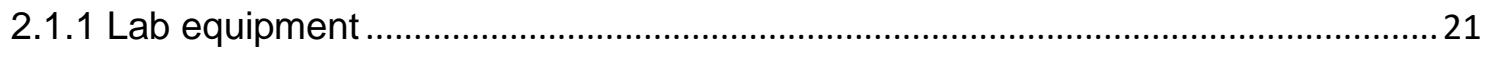

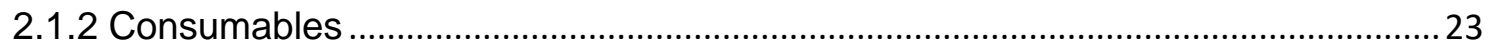

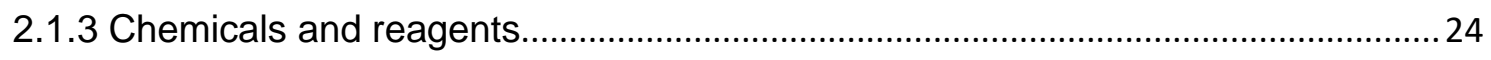

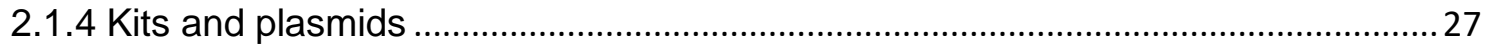

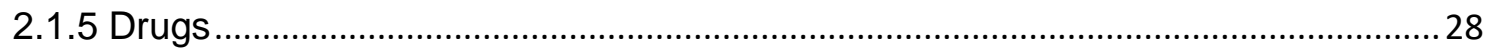

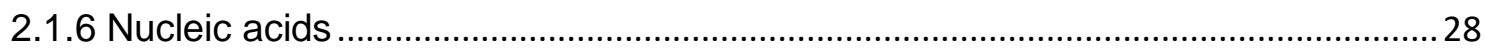

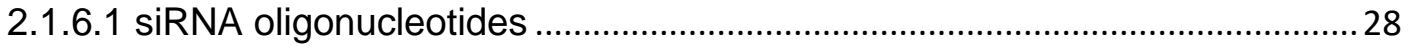

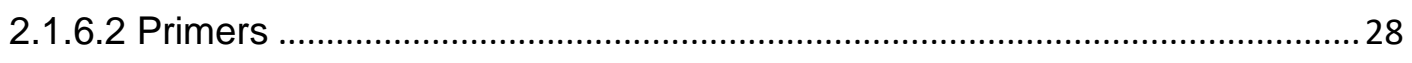

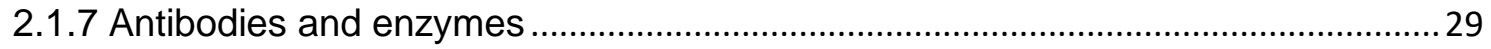

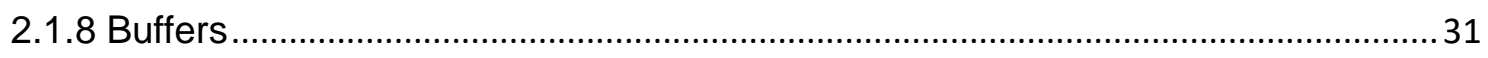




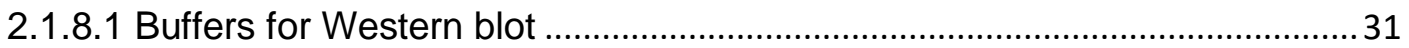

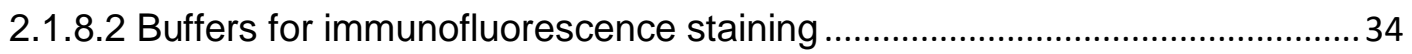

2.1.8.3 Buffers for Chromatin Immuno-Precipitation.................................................. 35

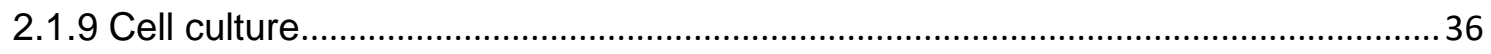

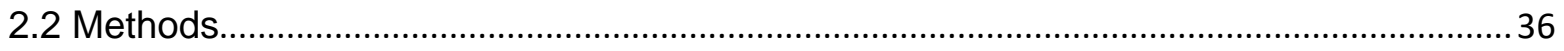

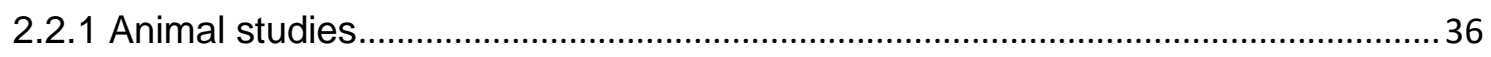

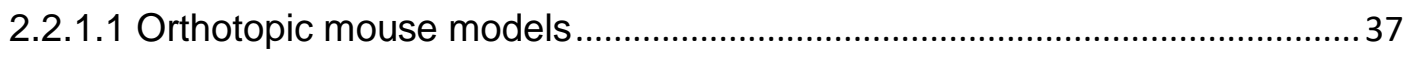

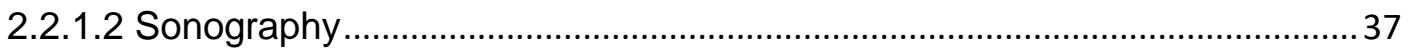

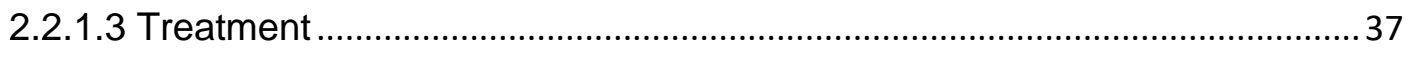

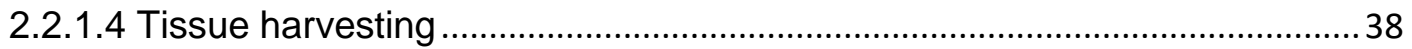

2.2.1.5 Paraffin embedding and tissue sectioning ................................................ 38

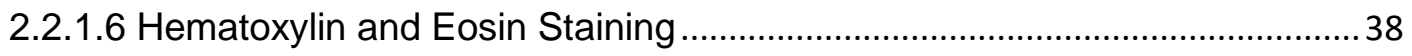

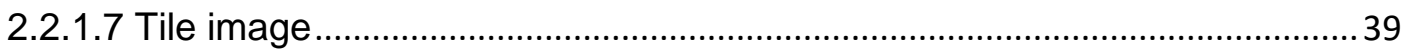

2.2.1.8 Immunofluorescence (IF) staining and quantification..................................39

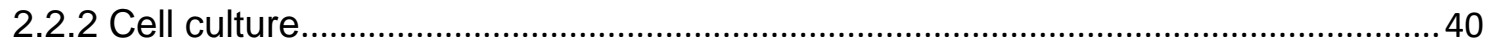

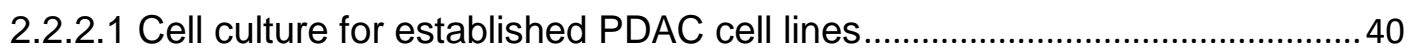

2.2.2.2 Generation of primary PDAC cells from patient-derived xenografts ........... 40

2.2.2.3 Generation of lentiviral and retroviral transduced stable cell lines ..............4 41

2.2.2.4 Cell viability assay for chemosensitivity experiments ................................. 42

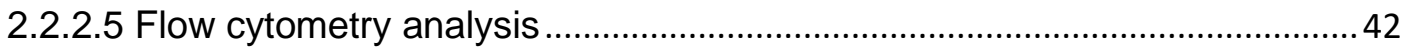

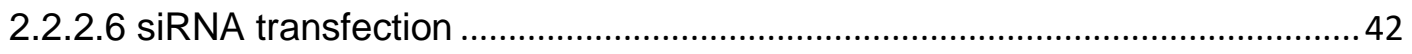

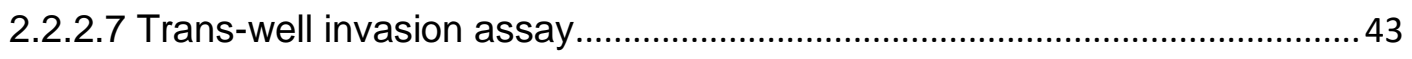

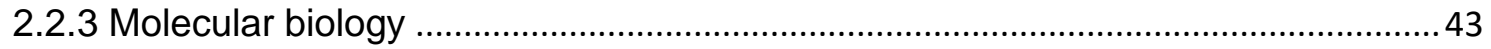

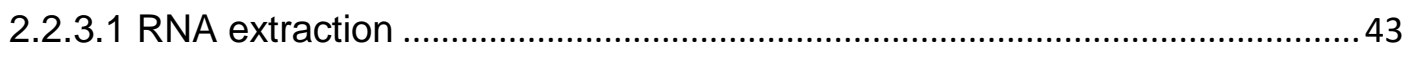

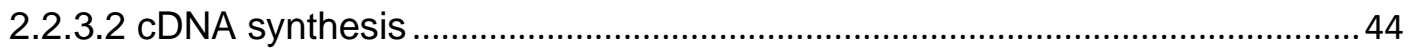

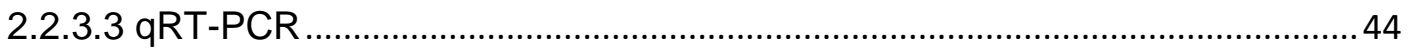

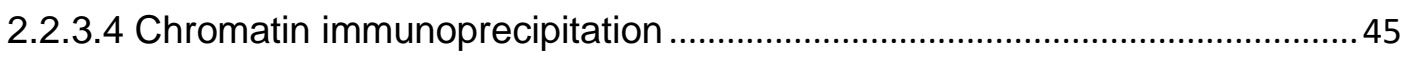

2.2.3.5 Protein sample preparation .......................................................................... 46

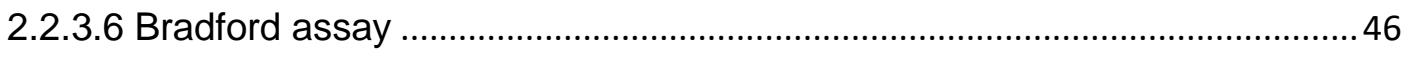




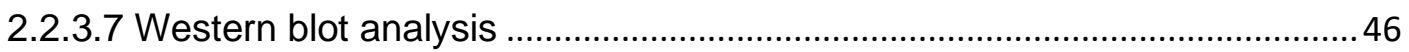

2.2.3.8 Proteome profiler cytokine array ............................................................ 47

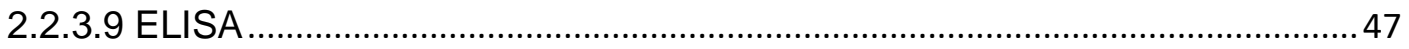

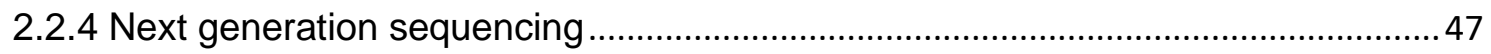

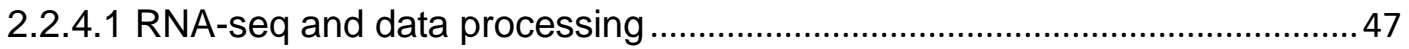

2.2.4.2 ChIP-seq library preparation and data processing .....................................48

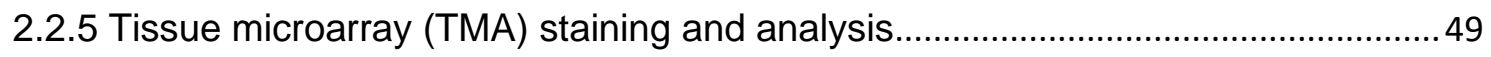

2.2.6 Publicly available PDAC microarray data analysis ..............................................49

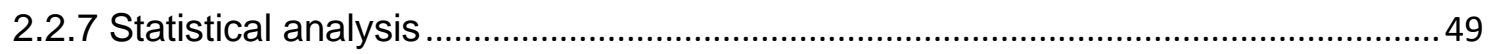

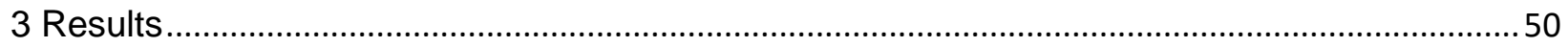

3.1 High level of TNFa is associated with BL phenotype in PDAC …...................................50

3.2 TNFa promotes CLA to BL phenotypic switch in PDAC .................................................52

3.2.1 TNFa promotes poorly differentiated phenotypic state in vivo ..............................52

3.2.2 TNFa promotes invasive behavior of CLA PDAC cells ........................................56

3.2.3 TNFa induces CLA to BL subtype switch by transcriptional reprogramming of

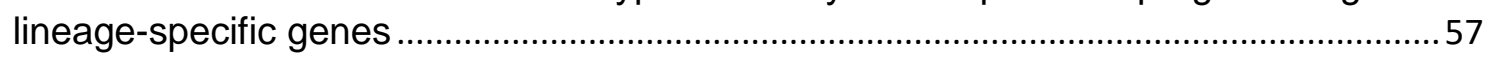

3.3 Molecular subtypes of PDAC determine distinct tumor microenvironment .....................60

3.3.1 BL-specific immune cells are source of TNFa in PDAC TME .............................60

3.3.2 Subtype-dependent immune cell infiltration ........................................................62

3.3.3 Subtype-specific intrinsic factors determine immune cell recruitment ..................63

3.4 Distinct AP1 transcription factors determine PDAC subtype identity ..............................65

3.4.1 Mutually exclusive expression of JUNB/AP1 and cJUN/AP1 in PDAC..................65

3.4.2 Differential genome binding profile of CLA-JUNB and BL-CJUN .........................67

3.4.3 cJUN overexpression induces invasiveness of CLA PDAC cells ..........................71

3.4.4 CJUN-CCL2 regulation in BL PDAC cells ............................................................. 73

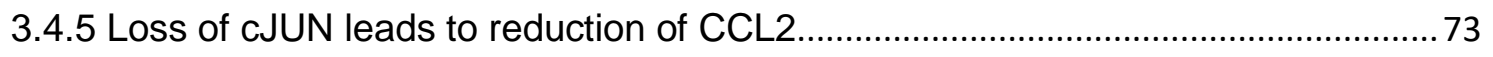

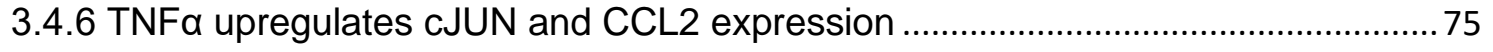

3.4.7 TNFa induces CCL2 expression via cJUN in PDAC ........................................... 76

3.5 TNFa monotherapy does not improve overall survival ..................................................77 
3.6 BRD4 controls cJUN expression via enhancer regulation

3.7 Pharmacological inhibition of the BRD4-cJUN-CCL2 axis supports a favorable

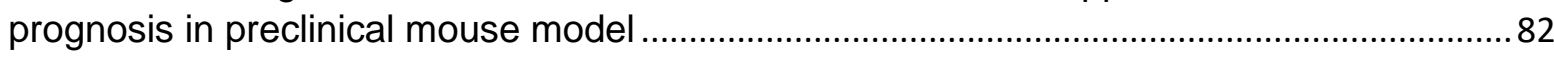

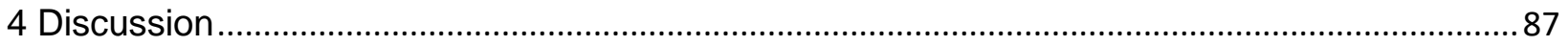

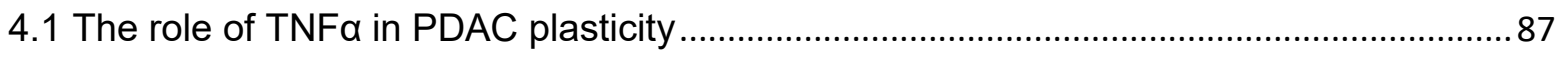

4.2 PDAC subtype-dependent stromal microenvironment...................................................90

4.3 The role of JUN/AP1 family in tumor plasticity .............................................................. 91

4.4 cJUN-regulatory network in macrophages recruitment and therapy resistance ..............93

4.5 Monotherapy of anti-TNFa is not ideal for PDAC …...................................................... 95

4.6 Preclinical inhibition of BRD4 confers favorable prognosis of BL tumors .......................96

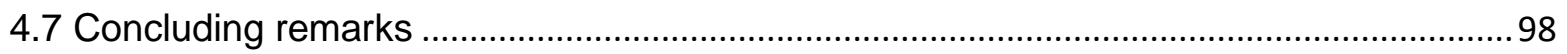

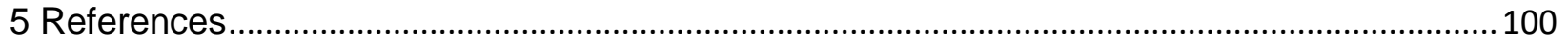

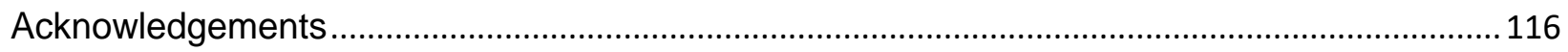




\section{List of figures}

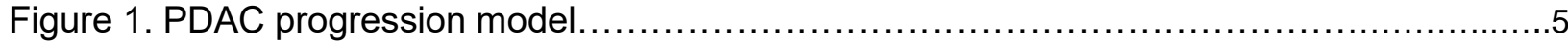

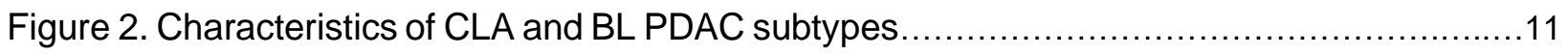

Figure 3. Proposed model of tumor-specific TFs in PDAC subtype maintenance …..................13

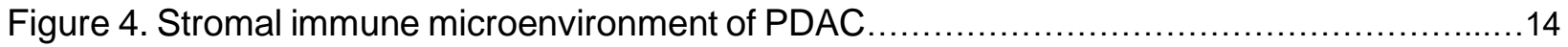

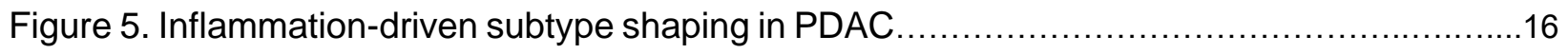

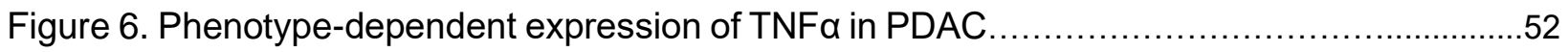

Figure 7. TNFa is highly expressed in poorly differentiated PDAC patient tumors......................53

Figure 8. Schematic of orthotopically implanted PDAC cells into the pancreas ..........................54

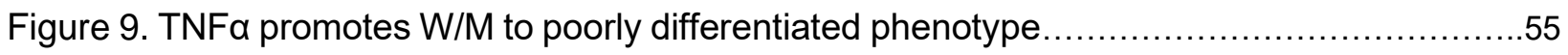

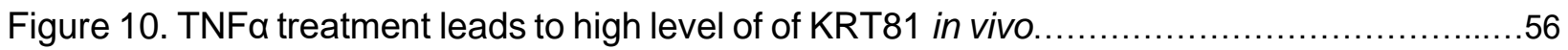

Figure 11. TNFa treatment induces CD45 immune cell infiltration..................................56

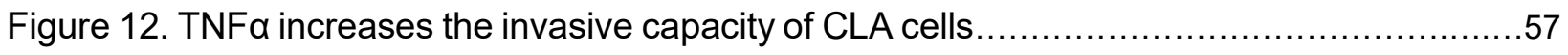

Figure 13 TNF $\alpha$ induced PDAC subtype-specific gene regulatory network...........................59

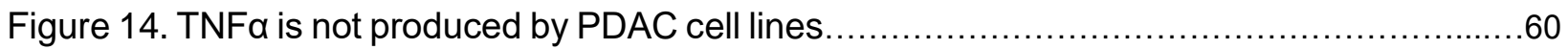

Figure 15. Host immune inflammatory tumor microenvironment produces TNFa in the BL PDAC

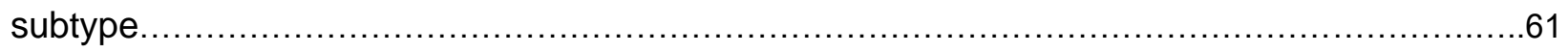

Figure 16. $\mathrm{CD}_{4} 5^{+}$and $\mathrm{CD} 68^{+}$cells are highly infiltrated in the BL orthotopic tumors...................62

Figure 17. CCL2 is linked to BL PDAC subtype and associates with CD68+ macrophages..........64

Figure 18. Subtype-restricted AP1 transcriptional program in PDAC ............................66

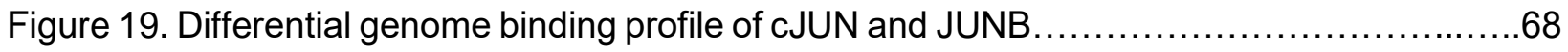

Figure 20. JUNB occupies $C D H 1$ active promoter and enhancer and loci whereas cJUN binds on VIM....... 


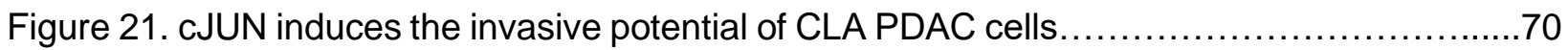

Figure 22. cJUN overexpression contributes to resistance to chemotherapeutics..................71

Figure 23. cJUN occupies active promoter and enhancer regions of CCL2 in BL cells............72

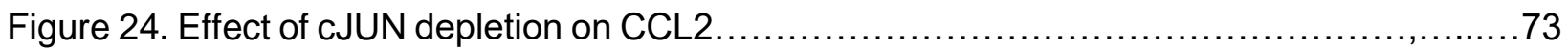

Figure 25. Exogenous TNFa treatment induces expression of cJUN and CCL2 in CLA cell

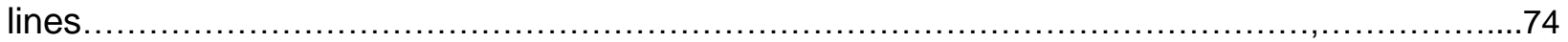

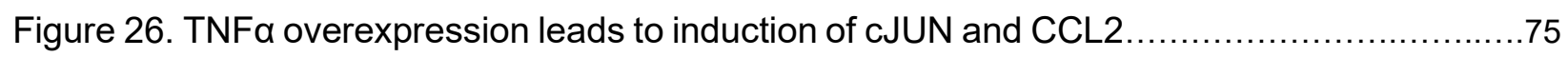

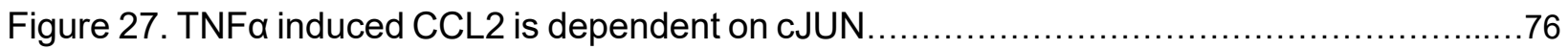

Figure 28. Inhibition of TNFa failed to improve the survival of syngeneic KPC mice...............77

Figure 29. Inhibition of TNFa did not alter tumor differentiation or infiltration of CD $45^{+}$cells.........78

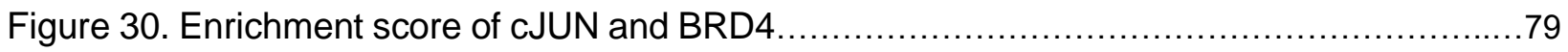

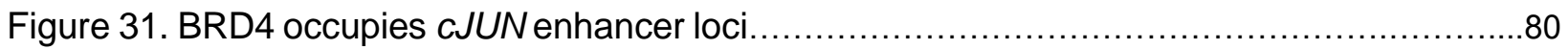

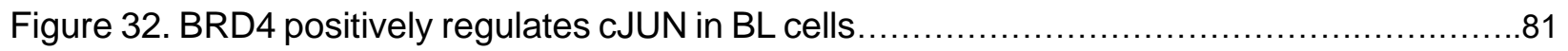

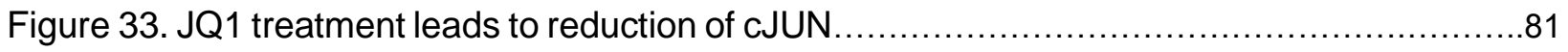

Figure 34. JQ1 resulted in a significant improvement of survival................................ 82

Figure 35. JQ1 treatment restrains tumor dedifferentiation $\ldots \ldots \ldots \ldots \ldots \ldots \ldots \ldots \ldots \ldots \ldots \ldots \ldots \ldots . . . \ldots 3$

Figure 36. JQ1 alone reduces inflammatory immune microenvironment and EMT factors........84

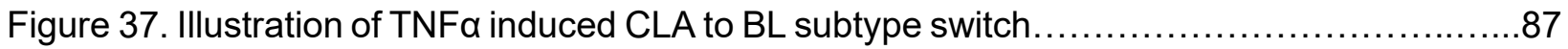

Figure 38. JUNB and cJUN in controlling PDAC subtype identity ..........................91

Figure 39. Illustration model of cJUN-CCL2-TNF $\alpha$ positive feedforward loop....................93

Figure 40. Schematic illustration of JQ1 mediated favorable prognosis in PDAC ................95 


\section{List of abbreviation}

5-FU 5-fluorouracil

ADEX Aberrantly differentiated endocrine exocrine

AGR2 Anterior Gradient 2

AP1 Activator protein 1

ATAC-seq Assay for transposase-accessible chromatin with sequencing

BSA Bovine serum albumin

BL Basal-like

BRD4 Bromodomain-containing protein 4

CAF Cancer-associated fibroblast

CCL2 CC-chemokine ligand 2

CDH1 Cadherin1

CDKN2A Cyclin dependent kinase inhibitor 2A

cDNA Complementary DNA

CEACAM5 Carcinoembryonic Antigen-Related Cell Adhesion Molecule 5

CEACAM6 Carcinoembryonic Antigen-Related Cell Adhesion Molecule 6

ChIP Chromatin immunoprecipitation

ChIP-seq ChIP followed by sequencing

CLA Classical

CO2 Carbondioxide

CSF1 Colony stimulating factor 1 
CSF1R Colony stimulating factor 1 receptor

DAPI 4',6-diamidino-2-phenylindole

DMEM Dulbecco's modified eagle medium

DMSO Dimethyl sulfoxide

DNA Deoxyribo nucleic acid

ECAD E-cadherin

ECM Extracellular matrix

EDTA Ethylenediaminetetraacetic acid

EGF Epidermal growth factor

EFGR Epidermal growth factor receptor

EGTA Ethylene glycol-bis ( $\beta$-aminoethyl ether)-N,N,N',N'-tetraacetic acid

ELF3 E74-like ETS transcription factor 3

EMT Epithelial to mesenchymal transition

FCS $\quad$ Fetal calf serum

FDA Food and drug administration

FOLFIRINOX Folinic acid, fluorouracil, irinotecan, oxaliplatin

Foxn1 ${ }^{\text {nu/nu }} \quad$ Homozygous nude mutant Forkhead box protein N1

FOXQ1 Forkhead Box Q1

GATA6 GATA-binding factor 6

GCDX Cell line derived from PDX established in Göttingen

GEMM Genetically engineered mouse models

GO Gene ontology 


\begin{tabular}{|c|c|}
\hline GSEA & Gene set enrichment analysis \\
\hline GTP & Guanidine triphosphate \\
\hline H3K4me3 & Histone 3 lysine 4 trimethylation \\
\hline H3K27ac & Histone 3 lysine 27 acetylation \\
\hline H3K27me3 & Histone 3 lysine 27 trimethylation \\
\hline HE & Hematoxylin \& eosin \\
\hline HNF4A & Hepatocyte Nuclear Factor 4 Alpha \\
\hline HRP & Horse raddish peroxidase \\
\hline$I_{50}$ & Inhibitory concentration $50 \%$ \\
\hline IF & Immunofluorescence \\
\hline $\lg G$ & Immunoglobulin G \\
\hline IGV & Integrative genomics viewer \\
\hline IL-1 & Interleukin-1 \\
\hline IL-6 & Interleukin-6 \\
\hline IL-10 & Interleukin-10 \\
\hline KDM6 & Lysine demethylase $6 \mathrm{~A}$ \\
\hline KLF5 & Krueppel-like factor 5 \\
\hline KPC & Kras ${ }^{G 12 D} ; \operatorname{Trp} 53^{R 172 H} ; P d x C r e$ \\
\hline KRAS & Kirsten rat sarcoma viral oncogene homolog \\
\hline KRT5 & Keratin 5 \\
\hline KRT14 & Keratin 14 \\
\hline LiCl & Lithium chloride \\
\hline
\end{tabular}




\begin{tabular}{ll} 
log2FC & Log2 fold change \\
mRNA & Messenger RNA \\
NaCI & Sodium chloride \\
NaF & Sodium fluride \\
NaO & Sodium oxide \\
NEAA & Nonessential amino acids \\
NES & Normalized enrichment score \\
NGS & Normal goat serum \\
PAGE & Polyacrylamide gel electrophoresis \\
PanIN & Pancreatic intraepithelial neoplasia \\
PARP & Poly-ADP ribose polymerase \\
PB & Phosphate buffer \\
PBT & Phosphate buffer with Triton X-100 \\
PBS & Phosphate-buffered saline \\
PCA & Principal component analysis \\
PCR & Polymerase chain reaction \\
PDAC & Pancreatic ductal adenocarcinoma \\
PDX & Patient-derive xenograft \\
PFA & Paraformaldehyde \\
PMSF & Phenylmethylsulfonyl fluoride \\
QM & Quasi-mesenchymal \\
qRCR & Quative real-time polymerase chain reaction \\
\hline
\end{tabular}




\begin{tabular}{|c|c|}
\hline RNA & Ribonucleic acid \\
\hline RNA-seq & RNA sequencing \\
\hline RPMI & Roswell Park Memorial Institute \\
\hline SD & Standard deviation \\
\hline SDS & Sodium dodecyl sulfate \\
\hline SDS-PAGE & Sodium dodecyl sulfate polyacrylamide gel electrophoresis \\
\hline SiRNA & Small interfering RNA \\
\hline SMAD & Mothers against decapentaplegic homolog \\
\hline TAM & Tumor associated macrophage \\
\hline TBS & Tris-buffered saline \\
\hline TBST & Tris-buffered saline with Tween-20 \\
\hline TCGA & The cancer genome atlas \\
\hline TEMED & Tetramethylethylenediamine \\
\hline TFs & Transcription factors \\
\hline TGF $\beta$ & Transforming growth factor $\beta$ \\
\hline TMA & Tissue microarray \\
\hline TME & Tumor microenvironment \\
\hline TNFa & Tumor necrosis factor $\alpha$ \\
\hline TP53 & Tumor protein 53 \\
\hline TRIS & Tris(hydroxymethyl)aminomethane \\
\hline VIM & Vimentin \\
\hline V & Volt(s) \\
\hline
\end{tabular}




$\begin{array}{ll}\mathbf{v} / \mathbf{v} & \text { Volume percent } \\ \mathbf{v s .} & \text { Versus } \\ \text { ZEB1 } & \text { Zinc finger E-box-binding homeobox } 1 \\ \boldsymbol{\alpha} & \text { Alpha } \\ \boldsymbol{\beta} & \text { Beta } \\ \boldsymbol{\mu} & \text { Micro }\end{array}$




\section{Abstract}

Pancreatic cancer (PDAC) represents an extremely poor clinical outcome with a 5-year survival rate of $<9 \%$. It is anticipated to become the second leading cause of cancerrelated deaths in the industrialized countries by 2030. A vast majority of PDAC patients exhibit locally advanced or distant metastases at the time of diagnosis, which makes surgical resection challenging. The complex molecular heterogeneity within neoplasticepithelium and stromal cells profoundly attributes to this poor prognosis, and makes therapy challenging. Extensive whole genome sequencing and transcriptional profiling of PDAC biopsies identified the two most clinically relevant and molecularly distinct subtypes: the basal-like $(\mathrm{BL})$ subtype displays highly aggressive phenotype, metastatic disease and chemoresistance profile in PDAC patients, whereas classical (CLA) subtype often responds to therapy and exhibits better prognosis. However, the coexisting stromal components (e.g. inflammatory macrophages and cancer-associated fibroblasts) within CLA or BL subtypes underlie distinct prognosis. Whether and how CLA or BL neoplastic cells shape the stromal microenvironment, and hence, determine PDAC aggressiveness and therapeutic vulnerabilities remain largely unresolved. Herein, we show that BL neoplastic cells recruit inflammatory macrophages, which foster highly inflamed and aggressive tumor phenotype in PDAC. We identified a mutually exclusive AP1-driven transcriptional program, which determines PDAC subtype identity and prognosis. CLArestricted JUNB/AP1 is associated with less aggressive and chemoresponsive CLA tumors; conversely, BL-restricted cJUN/AP1 largely controls tumor invasiveness, chemoresistance and proinflammatory program. Mechanistically, cJUN controls CCL2 expression via enhancer-promoter regulation, which, in turn, facilitate recruitment of TNFa-producing macrophages in the PDAC microenvironment. Subsequently, TNFa switches PDAC subtype identity through converting CLA tumors into a highly aggressive BL phenotypic state by activating CJUN-CCL2 signaling axis, thus, forming a positive feed forward loop. Finally, we show that BRD4 regulates cJUN-transcription via enhancerpromoter interactions; hence, pharmacological inhibition of the BRD4-cJUN axis induces a favorable subtype switch and improves overall survival in preclinical models. This study 
provides compelling evidence that subtype-specific transcriptional program shapes the subtype identity, tumor aggressiveness and prognosis in PDAC. Thus, cJUNhigh/TNFa ${ }^{\text {high }}$ subtype-specific precision therapy has the potential to overcome the highly aggressive and chemoresistant PDAC. 


\section{Introduction}

\subsection{Pancreatic cancer}

Pancreatic cancer (PDAC) is a deadly disease, which has the highest mortality rate among solid tumors with a 5-year survival rate of less than $9 \%$ (Siegel et al., 2020). According to the record of incidence and mortality associated with PDAC, it is set to become the second leading cause of cancer-related death by 2030 (Rahib et al., 2014). In comparison to breast and lung cancer, where treatment and early detection approaches have led to a significant improvement in prognosis (Andre and Pusztai, 2006; Rudin et al., 2019; Tsao et al., 2015), PDAC has an invariably poor prognosis since there is no biomarkers available for the early diagnosis. Besides, PDAC is highly aggressive and metastatic, showing strong resistance to chemotherapies. One of the major biomedical challenge in the poor prognosis and treatment of PDAC is the complex tumor stroma heterogeneity. However, factors involved in promoting cross-talk between tumor and stromal cells in the microenvironment remain unresolved.

\subsubsection{Pathophysiology}

The pancreas is a soft and glandular organ that mainly consists of endocrine and exocrine functional units (Zhou and Melton, 2018). The endocrine pancreas is responsible for regulating metabolism in the body, for example by regulating the blood glucose level. The exocrine compartment consists of acinar and ductal cells, where the acinar cells secrete the digestive enzymes (e.g. amylase, lipases, and proteinases) and ductal cells transport the secretion of enzymes to the small intestine (Zhou and Melton, 2018). In general, there are rare tumors arising from the endocrine pancreas; however, mainly neuroendocrine tumors (Kleeff et al., 2016). Over $90 \%$ of pancreatic tumors originate from the exocrine pancreas, with pancreatic ductal adenocarcinoma (PDAC) being the predominant type of tumors (Hezel et al., 2006; Prinz, 2012). Most PDAC arise from the head of the pancreas (60\%), some arise from the body (15\%) and tail (5-10\%) (Ghaneh et al., 2008). 


\subsubsection{Tumor initiation and progression}

The well-accepted PDAC progression model states that PDAC gradually develops from transformation of normal ductal epithelial cells to aggressive adenocarcinoma with the activation of certain oncogenes sequentially through the formation of well-defined precursor lesions called pancreatic intraepithelial neoplasia (PanIN-1 to 3) (Figure 1) (Hruban et al., 2001). PanIN-1 are lesions of flat or papillary architecture without any abnormalities. PanIN-2 lesions show enlarged and crowed nuclei, mild dysplasia with increased abnormalities and papillary architecture. PanIN-3 displays severe nuclear atypia, a high grade of dysplasia on the verge of carcinoma (Hruban et al., 2001).

In PDAC, KRAS mutations are the prevalent alterations in more than $90 \%$ cases (Olive and Tuveson, 2006). Further, mutations in KRAS are one of the earliest genetic events, which is essential for the initiation and development of PDAC (Kanda et al., 2012; Hingorani et al., 2003). KRAS protein is a Guanosine triphisphatase (GTPase), which is part of RAS/MAPK pathway (Iguchi et al., 2016). KRAS mutation leads to continuously binding of GTP in regardless of external stimulus and therefore promote tumor cell growth (Vigil et al., 2010; Waters and Der, 2018). Besides KRAS mutations, TP53, CDKN2A and SMAD4 are also considered as the most important driver mutations, which occur, in pancreatic carcinogenesis (Kleeff et al., 2016). For instance, CDKN2A encodes p16INK4A protein that binds and inactivates CDK4, leading to cell cycle G1 arrest (McWilliams et al., 2011; Zhao et al., 2016). On the other hand, CDKN2A encodes p16ARF for stabilizing p53 (Bartsch et al., 2002). Alterations in CDKN2A are observed in PanIN-2 lesions and more than $90 \%$ of PDAC tumors (Wilentz et al., 1998). TP53 is inactivated in more than $70 \%$ of PDAC (Puleo et al., 2018; Chan-Seng-Yue et al., 2020). Loss of TP53 favours tumor cell proliferation and survival as well as promoting additional unfavourable genetic alterations (Koorstra et al., 2009). Similarly, tumor suppressor SMAD4 is altered in more than $55 \%$ late stage PDAC (lacobuzio-Donahue, 2012). Mechanistically, SMAD4 is involved in transforming growth factor (TGF- $\beta$ ) mediated signaling pathway (lacobuzio-Donahue, 2012; Xia et al., 2015). During the progression of PDAC, pancreatic tissue is highly inflamed with cytokines e.g. TGF- $\beta$ (Shen et al., 2017). 


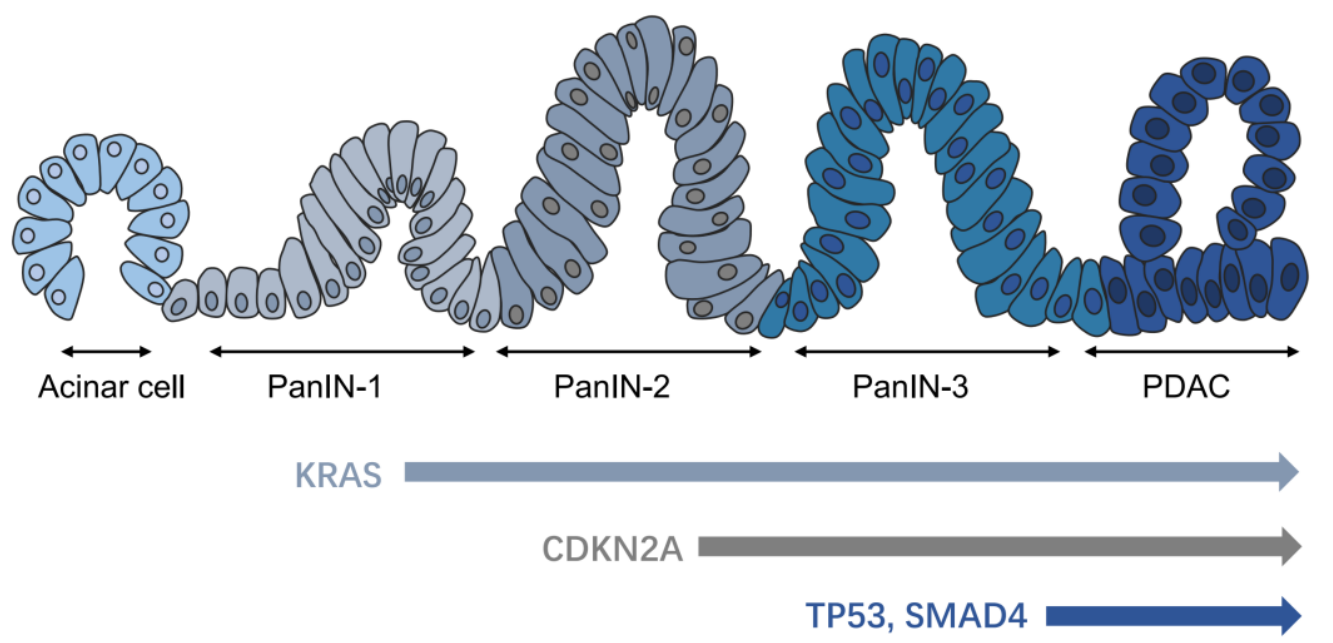

Figure 1. PDAC progression model. Illustration diagram of PDAC development from normal pancreatic tissue to PDAC through PanIN lesions (PanIN-1 to PanIN-3) and sequential activation of driver mutations KRAS, CDKN2A, TP53 and SMAD4.

\subsubsection{Therapeutic strategies of PDAC}

Due to the highly aggressive nature of PDAC, surgery is the only potentially curative therapeutic option (Hartwig et al., 2013). However, majority of patients are not amenable to surgery due to locally advanced or metastatic PDAC at the time of diagnosis. In fact, a large number of PDAC patients faced tumor recurrence post-surgical resection (Onoue et al., 2004). Nevertheless, the therapeutic strategies for PDAC have improved in the last decades, which has led to an increased 5-year survival from $11 \%$ to $21 \%$ post-surgery and chemotherapy (Strobel et al., 2019).

Gemcitabine is the most commonly used chemotherapeutic drug in the clinical settings for the treatment of PDAC patients (Burris et al., 1997). At the biochemical level, deoxycytidine, which is an active molecule of gemcitabine, converts into active metabolite that interferes with DNA synthesis and consequently inhibits tumor growth (Mini et al., 2006). Notably, American Society of Clinical Oncology (ASCO) recommended gemcitabine, together with capecitabine for resectable tumors (Khorana et al., 2017). A clinical study by the European Study Group for PDAC (ESPAC)-4 reported that the overall 
5-year survival rate of resected PDAC patients improved from 25.5 to 28.0 months with gemcitabine and capecitabine combined therapy compared to gemcitabine alone (Neoptolemos et al., 2017). However, the clinical outcome of adjuvant chemotherapeutic agents for the borderline resectable or unresectable tumors remain disappointing. Thus, neoadjuvant therapy emerged as an option for the borderline resectable or unresectable tumors, i.e. FOLFIRINOX treatment led to a conversion from unresectable stage to borderline or even resectable tumors (Nitsche et al., 2015; Petrelli et al., 2015).

For unresectable patients, chemotherapies remain the only option. For instance, combination of gemcitabine and nab-paclitaxel clinically applied as the first-line therapy for PDAC patients (Goldstein et al., 2015). An alternative to gemcitabine plus nabpaclitaxel for clinically fit patients is the FOLFIRINOX regimen, which is a combination therapy consisting of 5-FU, leucovorin, irinotecan, and oxaliplatin (Vaccaro et al., 2011). Notably, this combination significantly improves the outcome of metastatic patients when compared with monotherapy gemcitabine (11.1 vs 6.8 months) (Conroy et al., 2011). A phase III clinical trial reported that nanoliposomal irinotecan in combination with 5-FU/FA significantly improved the survival rate (6.1 vs 4.2 month) of those metastatic PDAC patients who had previously received gemcitabine-based therapy (Wang-Gillam et al., 2016). It is worth noting that, the second-line therapeutic option is determined based on the treatment applied in the first-line therapy as well as the patient performance (Ellenrieder et al., 2016).

Recently, the phase III POLO clinical trial reported that PARP inhibitor Olaparib improved progression-free survival. It is now recommended for the patients with germline BRAC mutation (Golan et al., 2019). Besides, immune therapy studies have shown significant improvement in survival rate in several cancer types. For instance, blockage of immune checkpoint T lymphocyte-associated antigen 4 (CTLA4) has been approved for clinical application in advanced melanoma (Hodi et al., 2010). Anti- programmed death 1 (PD1) and PD1 ligand (PDL-1) therapies were reported to be successfully established in multiple cancers including melanoma, lung cancer, and kidney cancer (Brahmer et al., 2015; Garon et al., 2015; Larkin et al., 2015; Motzer et al., 2015; Topalian et al., 2014). Nonetheless, PDAC is refractory to all these approaches, probably due to the negative 
immune regulation in the pancreatic tumor microenvironment (TME) (Neoptolemos et al., 2018).

Taken together, it is still very challenging to treat PDAC. Hence, extensive studies in the molecular subtyping are urgently needed to understand aggressive features and chemoresistance of PDAC. As in breast and lung cancer, subgrouping patients by molecular characteristics may benefit PDAC patients through optimized/personalized therapeutic approaches (Andre and Pusztai, 2006; Rudin et al., 2019; Tsao et al., 2015).

\subsection{Molecular subtypes of PDAC}

In recent years, considerable progress has been made in identifying molecular subtypes of PDAC, which remarkably improved the molecular insights of this grave disease (Table 1). In 2011, Collisson et al. defined three molecular subtypes of PDAC known as classical (CLA), quasi-mesenchymal (QM) and exocrine-like subtypes (Collisson et al., 2011), which is a landmark study that contributes to uncover the heterogeneity of PDAC. By performing microarray gene expression analysis in resected PDAC patient tumors, they identified a 62 gene signatures that allows clustering of tumors into certain subtypes (Collisson et al., 2011). The CLA subtype is found to be associated with high levels of epithelial gene signatures, while QM subtype has a high expression of mesenchymal genes and exhibit poor survival rate compared to CLA subtype. Multiple human and murine PDAC cell lines were able to delineate the CLA and QM subtypes (Collisson et al., 2011), which, in future, could be utilized as a model for studying subtype-specific therapeutic strategies.

After 4 years, another work in PDAC subtyping was conducted by Moffitt et al. (Moffitt et al., 2015). In their study, stroma compartment was taken into consideration and virtual microdissection approach was utilized in primary and metastatic tumors to distinguish between stromal and tumor-specific gene sets. Tumor epithelial compartment can be characterized into classical (CLA) and basal-like (BL) subtypes, which have been shown to overlap with the previous Collisson subtypes (Collisson et al., 2011). In contrast to the CLA subtype, BL subtype has worse prognosis (Moffitt et al., 2015). Besides molecular 
subtyping of epithelial-tumor compartments, Moffitt et al. molecularly characterized stromal compartments into two different subtypes: the normal and activated stroma. The so-called normal stroma corresponds to better prognosis, while activated stroma shows inflammatory signature and links to poor prognosis. In comparison to Collisson et al., the Moffitt et al. study has higher prognostic relevance since stroma subtypes were included and showed distinct tendencies.

In 2016, Bailey et al. performed RNA sequencing (RNA-seq) analysis in 96 bulk tumors to explore the transcriptional network and revealed four subtypes called progenitor, immunogenic, aberrantly differentiated endocrine exocrine (ADEX), and squamous (Bailey et al., 2016). The progenitor subtype is linked to the transcriptional programs, which are essential for endoderm fate determination. The immunogenic subtype shares high similarities with progenitor, and regulates pathways involving in antigen presentation and $B$ cell signaling. While ADEX tumors exhibit molecular signatures associated with pancreatic differentiation and development (Bailey et al., 2016). The most aggressive squamous subtype is associated with metabolic reprogramming, hypoxia, as well as inflammation. Furthermore, The Cancer Genome Atlas (TCGA) research network confirmed a significant overlap of the squamous to the BL subtype (Cancer Genome Atlas Research Network, 2017), previously identified by Moffitt et al. Besides, previously defined CLA subtype (Collisson et al., 2011 and Moffitt et al., 2015) that largely overlapped with the progenitor subtype (Bailey et al., 2016). However, ADEX and immunogenic subtypes were found to have less tumor purity, i.e. a high degree of contamination with non-neoplastic cells.

Puleo et al. conducted a microarray analysis in a large cohort of patient samples from 309 paraffin embedded PDAC tissues (Puleo et al., 2018). An unsupervised analysis concluded five subtypes: pure classical, immune classical, desmoplastic, stroma activated, and pure basal-like. With a high cellularity, tumors were classified into pure classical and pure basal-like subtypes. Notably, they identified a significant association of pure classical to well differentiated/low grade tumors. In contrast, pure basal-like subtype is linked to poorly differentiated/high grade tumors. The overall survival rate of pure basal-like patients represent significantly lower compared to pure classical subtype. 
In addition, most recent work from Chan-Seng-Yue et al. suggested the intra-subtype heterogeneity within the previously existing CLA and BL PDAC subtypes (Chan-SengYue et al., 2020). Overall, this study overcomes the limitation of the previous studies that only have access to resected tumor tissues where the genomic signatures from highgrade tumors or advanced stage tumors could be underrepresented. The transcriptomic based analysis of the primary and metastatic PDAC tumors identified classical-A, classical-B, hybrid, basal-like A, and basal-like B subtypes (Chan-Seng-Yue et al., 2020). Interestingly, this study suggested that basal-like A subtype is the most aggressive phenotype, which occurs in very late stage tumors, conferring high resistance to chemotherapy and consequently worse prognosis. Basal-like $B$ was found predominantly in the resectable PDAC tumors. However, CLA and BL subtypes were coexisted intratumorally (Chan-Seng-Yue et al., 2020), suggesting the highly heterogeneous phenotype of PDAC.

Table 1. PDAC subtypes.

\begin{tabular}{|c|c|c|c|c|}
\hline Study & Samples & Methodology & Subtypes & Significance/implications \\
\hline $\begin{array}{l}\text { Collisson et } \\
\text { al. } 2011\end{array}$ & $\begin{array}{l}63 \text { resected } \\
\text { PDAC tumors } \\
\text { (27 } \\
\text { microdissected; } \\
35 \text { whole } \\
\text { PDAC), and } 19 \\
\text { PDAC cell lines }\end{array}$ & $\begin{array}{l}\text { microdissection } \\
\text { transcriptome } \\
\text { (microarray) }\end{array}$ & $\begin{array}{l}\cdot \text {-Classical } \\
\cdot \text { QM } \\
\cdot \text { Exocrine-like }\end{array}$ & $\begin{array}{l}\text {-QM has poor survival } \\
\text { compared to classical } \\
\text { subtype }\end{array}$ \\
\hline $\begin{array}{l}\text { Moffitt et al. } \\
2015\end{array}$ & $\begin{array}{l}206 \text { tumors (145 } \\
\text { primary; } 660 \\
\text { metastatic), and } \\
134 \text { normal } \\
\text { pancreas }\end{array}$ & $\begin{array}{l}\text { Virtual } \\
\text { microdissection } \\
\text { transcriptome } \\
\text { (microarray) }\end{array}$ & $\begin{array}{l}\text {-Tumor compartment: } \\
\text { classical and basal-like } \\
\text { •Stromal compartment: } \\
\text { activated and normal }\end{array}$ & $\begin{array}{l}\text {-Basal-like and activated } \\
\text { stroma subtype are } \\
\text { correlated with worse } \\
\text { prognosis } \\
\text {-Basal-like tumors seems to } \\
\text { benefit more from adjuvant } \\
\text { therapy }\end{array}$ \\
\hline $\begin{array}{l}\text { Bailey et al. } \\
2016\end{array}$ & $\begin{array}{l}266 \text { primary } \\
\text { PDAC tumors }\end{array}$ & $\begin{array}{l}\text { Transcriptome } \\
\text { (RNA-seq) }\end{array}$ & $\begin{array}{l}\text {-Pancreatic progenitor } \\
\text {-Immunogenic } \\
\text { - ADEX } \\
\text {-Squamous }\end{array}$ & $\begin{array}{l}\text { - Squamous is associated } \\
\text { with poor survival }\end{array}$ \\
\hline
\end{tabular}




\begin{tabular}{|c|c|c|c|c|}
\hline TCGA 2017 & $\begin{array}{l}150 \text { PDAC } \\
\text { tumors }\end{array}$ & $\begin{array}{l}\text { Transcriptome } \\
\text { (RNA-seq) }\end{array}$ & $\begin{array}{l}\text { Overlap of existed } \\
\text { subtypes } \\
\text { (high-purity tumors): } \\
\text { •Classical/progenitor } \\
\text {-Basal-like/ } \\
\text { squamous }\end{array}$ & $\begin{array}{l}\text { - Inhibition of MTOR pathway } \\
\text { might be beneficial for } \\
\text { KRAS wild-type patients }\end{array}$ \\
\hline $\begin{array}{l}\text { Puleo et al. } \\
2018\end{array}$ & $\begin{array}{l}309 \text { resected } \\
\text { primary PDAC } \\
\text { tumors }\end{array}$ & $\begin{array}{l}\text { Transcriptome } \\
\text { (microarray) }\end{array}$ & $\begin{array}{l}\text {-Pure classical } \\
\text {-Immune classical } \\
\text {-Desmoplastic } \\
\text {-Stroma activated } \\
\text {-Pure basal-like }\end{array}$ & $\begin{array}{l}\text {-Pure basal-like is } \\
\text { associated with poor } \\
\text { survival }\end{array}$ \\
\hline $\begin{array}{l}\text { Chan-Seng- } \\
\text { Yue et al. } \\
2020\end{array}$ & $\begin{array}{l}248 \text { PDAC } \\
\text { tumors (primary } \\
\text { and metastatic) }\end{array}$ & $\begin{array}{l}\text { Laser capture } \\
\text { microdissection } \\
\text { transcriptome } \\
\text { (RNA-seq) }\end{array}$ & $\begin{array}{l}\cdot \text { Classical-A } \\
\cdot \text { Classical-B } \\
\cdot \text { Hybrid } \\
\text {-Basal-like-A } \\
\text {-Basal-like-B }\end{array}$ & $\begin{array}{l}\text {-Basal-like-A is highly } \\
\text { resistant to chemotherapy, } \\
\text { associated with extremely } \\
\text { poor prognosis }\end{array}$ \\
\hline
\end{tabular}

ADEX, Aberrantly differentiated endocrine exocrine; QM, Quasi-mensenchymal; RNA-seq, RNA sequencing; TCGA, The Cancer Genome Atlas.

Altogether, these transcription-based studies suggest the two most clinical-relevant PDAC molecular subtypes: the CLA subtype, characterized by expression of epithelial markers and corresponding to favorable prognosis, and the BL subtype, associated with high levels of epithelial-to-mesenchymal (EMT) signatures and correlating to unfavorable prognosis (Figure 2). Indeed, these subtypes show different vulnerabilities to chemotherapy: in particular, BL tumors show a high degree of chemoresistance, promoting a poor patient prognosis. The COMPASS trial reported by Aung et al. showed that the CLA subtype patients respond better to either modified FOLFIRINOX or gemcitabine/ plus nab-paclitaxel chemotherapy, while BL subtype still continuously progress under treatment (Aung et al., 2018). 
CLA subtype

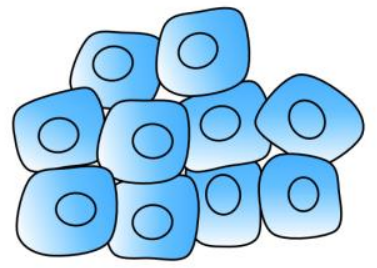

- Well differentiated

- Chemoresponsive

- Better prognosis
BL subtype

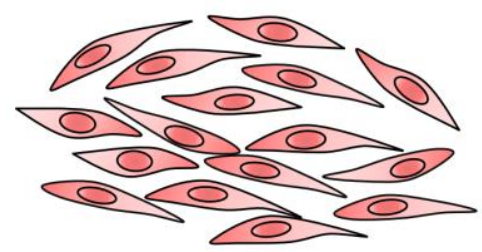

- Poorly differentiated

- Chemoresistant

- Poor prognosis

Figure 2. Characteristics of CLA and BL PDAC subtypes. PDAC can be robustly characterized into two subtypes: CLA and BL subtype. They display distinct tumor histology features, chemoresistance as well as prognosis.

\subsection{Subtype-specific regulatory network}

Despite having defined the prognostically relevant CLA and BL subtypes, the regulatory networks within tumor cells, especially the key transcription factors (TFs) that control subtype-specific gene programs and maintain their cellular identity, remain largely unknown. An initial study comprehensively addressed the subtype-specific transcriptional regulation as well as control of lineage-specific markers in PDAC phenotypic identity (Diaferia et al., 2016). By performing RNA-seq analysis in established human PDAC cell lines, they identified a set of genes corresponding to high- and low-grade tumors, reflecting CLA and BL tumors respectively. Among those, KLF5, ELF3, HNF1B, as well as JUNB were mainly expressed in low-grade tumors. In contrast, high-grade tumors showed abundant expression of ZEB1, GATA2 and ETV5 (Diaferia et al., 2016). Besides these grade-specific markers, 'GATA6' emerged as a potential biomarker for the CLA subtype identity in PDAC patient tumors (Aung et al., 2018; Chan-Seng-Yue et al., 2020). PDAC patients with high-grade tumors expressed significantly low GATA6, supporting by RNA in situ hybridization assay in the tumors (Aung et al., 2018). Further, to determine the role of GATA6 in the regulation of cell identity, Martinelli et al. employed shRNA- 
mediated GATA6 silencing in multiple low-grade PDAC cell lines (Martinelli et al., 2017). Intriguingly, genetic inactivation of GATA6 led to loss of epithelial-lineage markers (e.g. E-cadherin) and gain of the EMT-related markers such as vimentin in the GATA6depleted PDAC cells. Furthermore, cells acquired significantly more invasive and BL phenotypic identity upon GATA6 silencing. Furthermore, patients with low expression of GATA6 have shorter survival and a poor response to chemotherapy compared to those who have medium or high levels of GATA6 (Martinelli et al., 2017, Aung et al 2018). Recently, HNF4A, a low-grade lineage TF found to be an essential player in maintaining CLA subtype identity (Brunton et al., 2020). NHF4A depleted cells showed induction of $B L$ phenotype gene signature, with upregulation of gene sets involved in WNT, insulin, as well as phosphatidylinositol 3-kinase (PI3K)-AKT signaling pathways. Besides, loss of HNF4A led to activation of BL-associated metabolic signatures (Brunton et al., 2020).

Overall, identification of low-grade/CLA-specific TF (i.e. GATA6) turned out to be a significant tool for PDAC patient stratification. Similarly, several studies have focused on deciphering the gene regulatory network underlying $\mathrm{BL}$ phenotype. Notably, it has recently been shown that TFs such as GLI2 and TP63 $(\Delta \mathrm{Np63})$ maintain BL subtype identity (Adams et al., 2019; Somerville et al., 2018). GLI2 alone is sufficient to cause a switch from CLA to BL subtype in PDAC cells (Adams et al., 2019). Similarly, TP63 was also identified as a key factor that controls a transcription regulatory network supporting the squamous/BL phenotypic state. Moreover, ectopic expression of TP63 was shown to be sufficient to induce the reprogramming of TFs for the maintenance of a squamous differentiation in PDAC (Somerville et al., 2018). Whether and how lineage-specific TFs recruit chromatin regulators to shape PDAC subtype plasticity remain poorly understood. Recent studies have shown that chromatin regulator lysine demethylase 6A (KDM6) is required to maintain the CLA PDAC subtype identity both in vitro and in vivo (Andricovich et al., 2018). Pancreas specific loss of Kdm6a (Kras-Kdm6a-/- mice) displayed poorly differentiated histological features. Mechanistically, global gene expression profile showed that depletion of Kdm6a led to enrichment of EMT, pro-inflammatory, and response to hypoxia pathways, corresponding to BL phenotypic identity (Andricovich et al., 2018). 
Altogether, these studies have provided evidence for the mechanisms of regulation of PDAC subtypes. Here, we propose a model of lineage TFs determined subtype identity (Figure 3). By exploring the specific lineage markers, it will be possible to exploit clinical diagnostic markers as well as tailored therapeutic approaches.
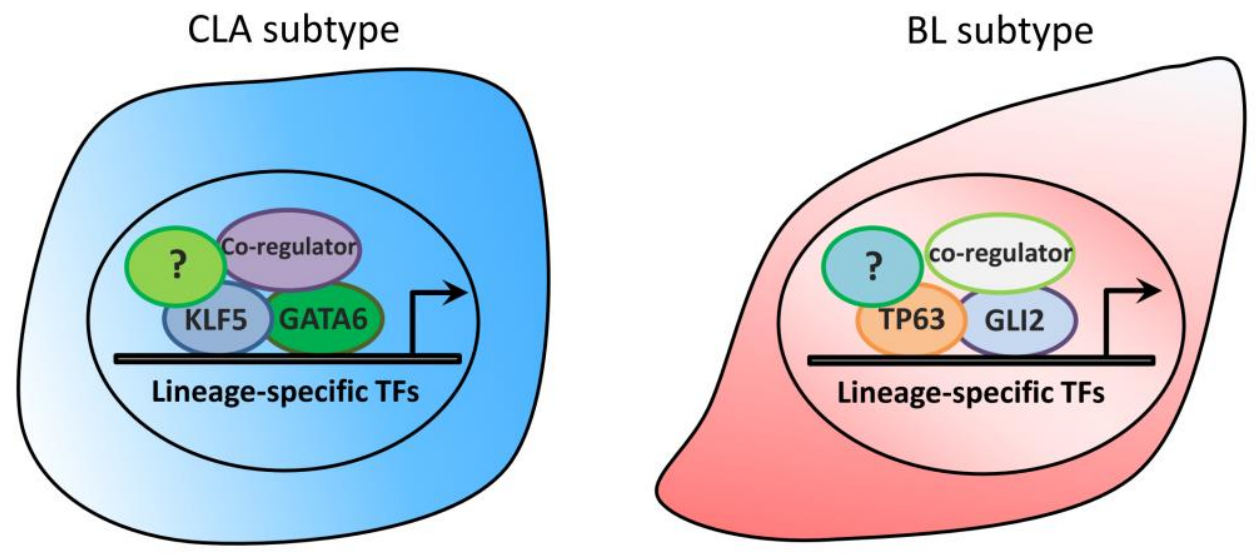

Figure 3. Proposed model of tumor-specific TFs in PDAC subtype maintenance. Lineagespecific TFs and coregulators shape CLA and BL subtype identity.

\subsection{The tumor microenvironment}

The TME of PDAC has a significant impact on tumor initiation, progression, and metastasis (Hessmann et al., 2020). PDAC has a complex TME that consists of endothelial cells, cancer-associated fibroblasts (CAFs), tumor cells, extracellular matrix (ECM), immune cells, and soluble factors such as growth factors, cytokine, and chemokine (Figure 4). CAFs and Tumor-associated macrophages (TAMs) are two prominent components of the inflammatory TME. In most of the solid tumors, the existence of TAMs is associated with an unfavorable prognosis (Komohara et al., 2014; Zhang et al., 2012). In PDAC, high expression of macrophage signatures are associated with poor prognosis (Bailey et al., 2016). Single-cell analysis performed by Hosein et al. revealed that the population of TAMs dramatically increased at the late stage of the disease compared to the initial phase, indicating the correlation between macrophage and aggressive PDAC phenotype (Hosein et al., 2019). Moreover, Candido et al. showed 
that macrophage represses $T$ cell activity to allow for tumor growth and is associated with the squamous phenotype (Candido et al., 2018). A subtype switch from squamous to progenitor could be seen upon macrophage depletion. In addition, the macrophage is found to inhibit the efficiency of gemcitabine by releasing pyrimidine nucleosides (Halbrook et al., 2019). Targeting macrophage via inhibitor against the colony stimulating factor 1 receptor (CSF1R) could restore the effect of gemcitabine (Candido et al., 2018).

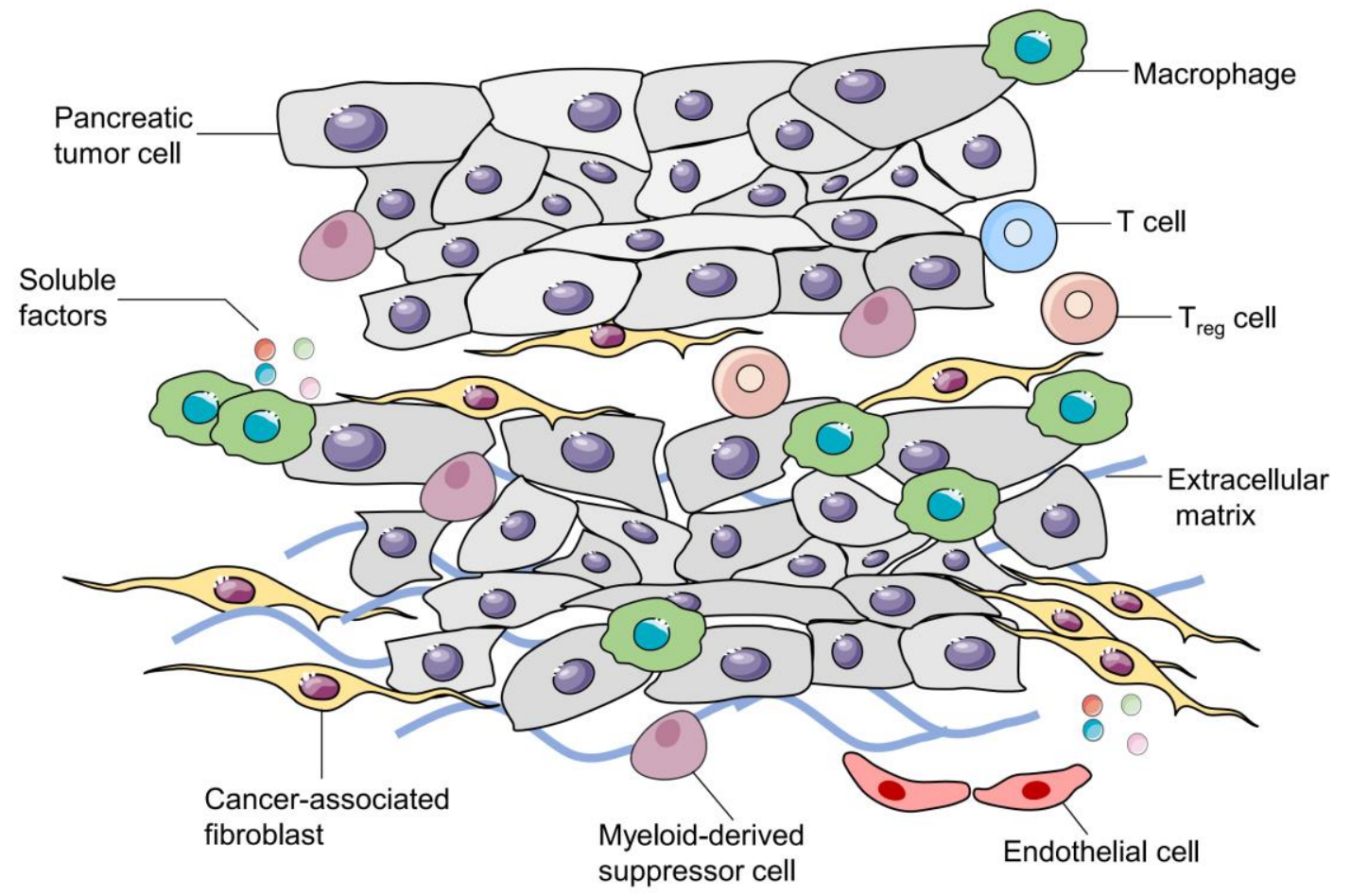

Figure 4. Stromal immune microenvironment of PDAC. The TME of PDAC comprises diverse cell types including tumor cell, endothelial cell, CAFs, extracellular matrix, soluble factors, as well as immune cells, such as $T$ cell, $T_{\text {reg }}$ cell, TAMs and myeloid-derived cell.

\subsubsection{Inflammatory tumor stroma microenvironment}

\subsubsection{Role of inflammation in tumor progression}

Inflammatory microenvironment is evidenced to be an essential content for almost all types of tumors (Mantovani et al., 2008). It is well established that inflammation-linked 
diseases increase the risk of cancer development, as in prostate cancer, ovarian cancer, gastric cancer, intestinal cancer, as well as bladder cancer (Grivennikov et al., 2010; Mantovani et al., 2008). Inflammation has a significant impact on PDAC tumor development and progression (Guerra et al., 2007; Rhim et al., 2012., Singh et al 2015). Apart from smoking and alcohol consumption, chronic pancreatitis is considered the main risk factor for developing PDAC (Yadav and Lowenfels, 2013). Inflammation is initially induced in the context of pancreatitis to prevent the loss of homeostasis and promote tissue regeneration. For example, acinar cells transform into duct-like architecture termed acinar-to-ductal metaplasia (ADM) in response to inflammatory cues (Liou et al., 2013). Normally, ADM is highly plastic and reversible to allow the repair of pancreatic injury (Rooman and Real, 2012; Stanger and Hebrok, 2013). However, in the context of cancer, with the constitutively activation of oncogene KRAS and chronic presence of inflammatory cytokines, acinar cell displays a high degree of abnormalities and develops into full-blown PDAC (Logsdon and Ji, 2009).

Rihm et al. have unveiled a significant role of inflammation in pancreatic tumor formation and dissemination (Rhim et al., 2012). In the metastatic PDAC mouse model, EMT and invasiveness were extensively surrounded by inflammatory cells. Cerulein-induced inflammation in mice resulted in accelerated formation of PanIN lesions and increased circulating cells. For the confirmation of inflammation induced EMT, mice received antiinflammatory drug, which showed a general reduction of inflammation and feebly developed PanIN lesions (Rhim et al., 2012). Based on these data, we proposed a model that the external stimulus (e.g. cytokines or chemokines) from the inflammatory microenvironment might induce PDAC subtype switch, which, in turn, lead to disease aggressiveness (Figure 5). 


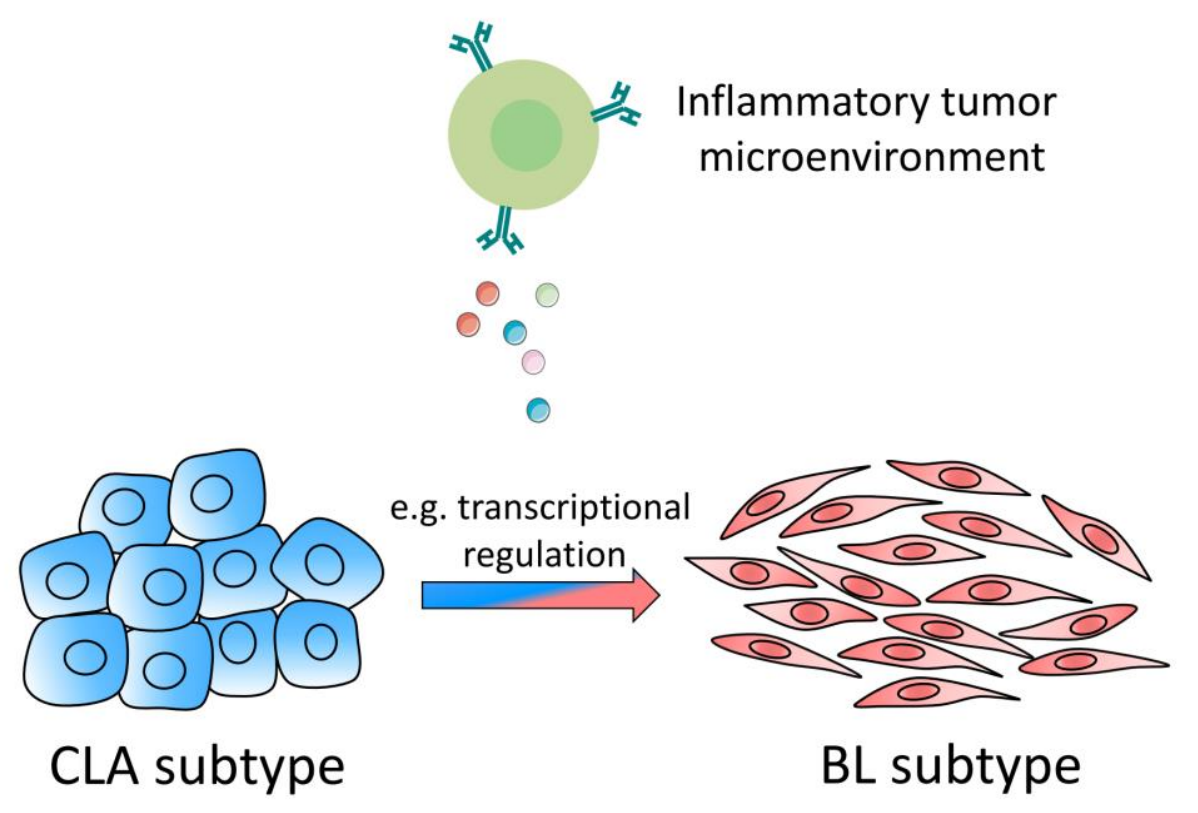

Figure 5. Inflammation-driven subtype shaping in PDAC. Inflammatory cytokines and chemokines secreted from the TME might induce formation of an aggressive subtype.

A recent study by Cobo et al. showed cell-autonomous reprogramming driven by inflammation that regulated the differentiation of acinar cells and contributed to the development of PDAC (Cobo et al., 2018). The orphan nuclear receptor NR5A2 is involved in pancreatic embryonic development and controls acinar cell differentiation (Hale et al., 2014). In PDAC patients, there is a strong correlation of low NR5A2 expression to the patients who had chronic pancreatitis previously (Cobo et al., 2018). In $\mathrm{Nr} 5 \mathrm{a} 2$ wild type mice, NR5A2 binds on genes that maintain acinar cell differentiation. Interestingly, NR5A2 shifts its binding from acinar-related genes to inflammatory genes in $\mathrm{Nr}_{5 \mathrm{a} 2^{+/}}$pancreata, via interaction with cJUN/AP-1 transcription factor. Caeruleintreated (induce pancreatitis) wild type mice also exhibited a similar inflammatory profile. Notably, inhibition of cJUN TF led to abrogation of this transcriptional switch, thus, inflammatory gene expression was halted. Mechanistically, NR5A2 cooperates with NrOb2 to represses cJUN activity to maintain low inflammation and favorable outcome (Cobo et al., 2018). Therefore, NR5A2 loss leads to high induction of cJUN and further facilitate the acinar-to-inflammatory switch, which leads to unfavorable outcome. 


\subsubsection{Tumor stroma microenvironment in disease progression}

Cytokines are key soluble factors in connecting inflammation to cancer (Grivennikov et al., 2010). They play a crucial role in modulating cellular components in TME, especially the interactions between epithelial and stromal immune compartment (Shadhu and Xi, 2019). In PDAC microenvironment, the dynamic cross-talk between tumor cells and inflammatory cells is largely determined by cytokines. Inflammatory cells (e.g. TAMs and CAFs) are the main source of cytokine production, secreting IL-1, IL-6, IL-10, TGF- $\beta$ and TNFa to influence tumor cell behavior and promote PDAC progression (Biffi et al., 2019; Huang et al., 2019; Ikemoto et al., 2000; Karakhanova et al., 2015; Zhao et al., 2016).

TNFa is a well-known master regulator of inflammation and immune response. It is a type II transmembrane protein and is released by the cleavage of a converting enzyme at the site between $\mathrm{Ala}^{76}-\mathrm{Val}^{77}$ (Wajant, 2009). TNFa can be produced in multiple cell types including macrophage, activated monocyte, activated T cell, dendritic cell, nature killer (NK) cell, microglia, as well as astrocyte (Zidi et al., 2010). TNFR1 (p55) and TNFR2 (p75) were identified as receptors for TNFa. TNFR1 is expressed ubiquitously on all cell types; however, TNFR2 is restricted to endothelial and immune cells (Sethi et al., 2008). While extensive efforts have been made to study the role of TNFa in regulating inflammation and inflammatory disease, the functional relevance of TNFa in tumor progression and drug resistance have not been fully addressed.

Lind et al. revealed a significant role of TNFR1 in skin cancer development (Lind et al., 2004). Squamous carcinoma mouse model develops rather quickly microscopically skin changes in 3 weeks, with massive immune cell infiltration. However, Tnfr $1 \%$ mice do not give rise to skin tumor and inflammation (Lind et al., 2004), suggesting that TNFR1mediated signaling pathway is involved in the development of skin cancer. In breast cancer, over half of the studied patients (105 patients in total) were found to have TNFa abundance in their tumor tissues characterized by immunohistochemistry staining. Tumor cells isolated from patients with high- and low- expression of TNFa were subjected to doxorubicin (DOX) treatment. It showed that TNFa-high expressing cells confer more resistance to DOX compared to TNFa-low expressing cell lines (Zhang et al., 2018). In 
PDAC, TNFa induces invasive capacities of the PDAC cells and secretions of IL-8 and MMP9 (Egberts et al., 2008). Administration of TNFa in vivo leads to increased tumor growth and metastasis. However, blocking TNFa in combination with chemotherapy reduces tumor volume and metastasis in PDAC models (Egberts et al., 2008). Moreover, Zhao et al. has reported that high levels of TNFa is associated with an unfavourable prognosis in PDAC patients (Zhao et al., 2016).

\subsubsection{Reciprocal interaction between stromal and tumors cells in PDAC prognosis}

In the TME, studies have shown that the co-existence of stromal cells within tumor cells linked to distinct prognosis in PDAC. Moffitt et al. showed that coexisting activated stroma within BL tumors are linked to poor prognosis (Moffitt et al., 2015). However, Puleo et al., showed that activated stroma within BL tumors led to better survival of PDAC patients (Puleo et al., 2018). Therefore, further studies are required to address the stromal immune determined PDAC subtype identity and prognosis. It is known that tumor cells are capable of changing their phenotype in response to certain inflammatory factors secreted by the stromal compartment, and vice versa (Mantovani et al., 2008). TME can determine the tumor cell differentiation, as implanting identical patient-derived organoids directly into ducts or interstitial space of the pancreas, which induces either an intraductal or a stroma TME, respectively (Miyabayashi et al., 2020). It turned out that the EMT marker vimentin (VIM) was highly elevated in the tumors that grow in a stromal TME compared to the ones grown in intraductal TME. Interestingly, those organoids were also showed to transform stromal cells to gain more invasive properties with the induction of EMT, and specifically, TGF- $\beta$ and WNT (Miyabayashi et al., 2020). Similarly, another study evidenced that TME shapes the phenotypic status of organoids regardless of the genotype (Raghavan et al., 2020). In addition, the soluble factors from TME induce cell identity shift concordant to gain of $\mathrm{BL}$ genes and loss of organoid-related genes (Raghavan et al., 2020).

In summary, these studies highlighted the importance of the dynamic interplay between stromal and epithelial compartments. How the two major PDAC subtypes act to shape 
and interact with its microenvironment remains poorly understood. Hence, molecular understanding of the heterotypic interactions within TME could provide a novel insight into the development of specific therapeutic strategies.

\subsection{Aims of the study}

Recent studies have invested tremendous efforts in molecular classification of PDAC. In particular, the transcriptional profiling of TME indicates a significant correlation of stroma inflammatory gene signatures to distinct clinical outcomes (Singh et al., 2019; Moffitt et al., 2015; Maurer et al., 2019; Puleo et al., 2018). Markedly, the stromal immune compartment within differential epithelial tumor subtypes determine the therapeutic response and prognosis in PDAC (Singh et al., 2019; Moffitt et al., 2015).

Inflammatory cytokines are mediators of the cross talk between tumor cells and microenvironment. For instance, TNFa expression has been shown to be negatively associated with PDAC patient survival (Zhao et al., 2016). Studies have shown that PDAC tumor cells can change the plasticity through transcriptional reprogramming particularly in response to inflammatory cue (Rhim et al., 2012; Singh et al 2015). Therefore, we proposed that TNFa might change the plasticity of PDAC cells and mediate subtype switch from CLA to BL to accelerate the progression of PDAC.

In this study, we aimed to characterize the role of TNFa in PDAC subtype switch as well as examine the underlying mechanisms and the potential of selective pathway interference in PDAC therapy. Our specific aims were as follow:

1. To investigate the role of TNFa in PDAC subtype maintenance and disease aggressiveness;

2. To elucidate the mechanism of TNFa-dependent transcriptional reprogramming in PDAC subtype switch;

3. To explore the intrinsic factors which contribute to PDAC subtype identity and aggressiveness;

4. To identify TNFa-induced subtype-specific chromatin regulators for pharmacological interventions; 
5. To evaluate the therapeutic interventions against TNFa-mediated inflammationregulatory network in a PDAC subtype-specific therapy. 


\section{Material and methods}

\subsection{Material}

\subsubsection{Lab equipment}

Table 2. Lab equipment.

\begin{tabular}{|c|c|}
\hline Equipment & Company \\
\hline Agarose electrophoresis chamber & Peqlab Biotechnologie, Erlangen, Germany \\
\hline Anesthetic vaporizer-Sigma delta & Penlon, Abingdon, UK \\
\hline Arium pro ultrapure water system & Sartorius, Göttingen, Germany \\
\hline Aspirator with trap flask (Grant bio FTA-1) & Grant Instruments, Cambridge, UK \\
\hline Bioanalyzer 2100 & Agilent Technologies, Santa Clara, USA \\
\hline Bioruptor Pico sonication device & Diagenode, Liege, Belgium \\
\hline Centrifuge-Perfectspin 24R (refrigerated) & Peqlab Biotechnologie, Erlangen, Germany \\
\hline $\begin{array}{l}\text { Centrifuge-Heraeus Megafuge } 16 \text { / } \\
\text { Multifuge X1R }\end{array}$ & Thermo Fisher Scientific, Waltham, USA \\
\hline $\mathrm{CO}_{2}$ incubator (HERAcell 240i) & Thermo Fisher Scientific, Waltham, USA \\
\hline Cold plate-Histocore Arcadia c & Leica Biosystem, Wetzlar, Germany \\
\hline Fluid Aspiration System - BVC Control & Vacuubrand, Wertheim, Germany \\
\hline Fluoview F1000 Confocal Microscope & Olympus, Shinjuku, Japan \\
\hline Freezer $4 \stackrel{\circ}{\mathrm{C}}$ & Liebherr, Bulle, Switzerland \\
\hline Freezer $-20^{\circ} \mathrm{C}$ & Liebherr, Bulle, Switzerland \\
\hline Freezer $-80^{\circ} \mathrm{C}$ & Sanyo Electric, Japan \\
\hline $\begin{array}{l}\text { Heated Paraffin Embedding Module - } \\
\text { EG1150 H with cold plate }\end{array}$ & Leica Biosystems, Nussloch, Germany \\
\hline gentleMACS dissociator & Miltenyi Biotec, Bergisch Gladbach, Germany \\
\hline Ice flaker-AF80 & Scotsman, Edinburgh, UK \\
\hline INTAS UV System & $\begin{array}{l}\text { Intas Science Imaging Instruments, Göttingen, } \\
\text { Germany }\end{array}$ \\
\hline $\begin{array}{l}\text { Liquid nitrogen cell storage canister- } \\
\text { Biocane } 47\end{array}$ & Thermo Fisher Scientific, Waltham, USA \\
\hline
\end{tabular}




\begin{tabular}{|c|c|}
\hline Light microscope "BX43" & Olympus, Shinjuku, Japan \\
\hline Microplate reader PHOmo / LUmo & Autobio Labtec Instruments, Zhengzhou, China \\
\hline Microscope-Axiovert 25 & Carl Zeiss, Oberkochen, Germany \\
\hline $\begin{array}{l}\text { Microwave heat pad for animals- } \\
\text { snuggleSafe }\end{array}$ & SnuggleSafe Lenric C21, West Sussex, UK \\
\hline Nano Photometer-P330 & Intas Science Imaging, Göttingen, Germany \\
\hline Nalgene Mr. Frosty freezing container & Thermo Fischer Scientific, Waltham, USA \\
\hline Neubauer counting chamber & Brand, Wertheim, Germany \\
\hline Paraffin tissue embedder (EG1150H) & Leica Biosystems, Wetzlar, Germany \\
\hline Perfect Spin 24Plus Microcentrifuge & VWR/Peqlab, Erlangen, Germany \\
\hline $\begin{array}{l}\text { Perfect Spin 24R Refrigerated } \\
\text { Microcentrifuge }\end{array}$ & VWR/Peqlab, Erlangen, Germany \\
\hline pH meter-FiveEasy Plus FEP20 & Mettler Toledo, Schwerzenbach, Switzerland \\
\hline Plate Spinner-PerfectSpin P & Peqlab Biotechnologie, Erlangen, Germany \\
\hline PowerPac HC High-Current Power Supply & Bio-Rad Laboratories, Hercules, USA \\
\hline Qubit Fluorometer-Q32854 & Invitrogen, Karlsruhe, Germany \\
\hline StepOnePlus real-time PCR system & Applied Biosystems, Foster City, USA \\
\hline Rotary Microtome-RM2265 & Leica Biosystems, Nussloch, Germany \\
\hline Shaker-Duomax 1030 & Heidolph Instruments, Schwabach, Germany \\
\hline Shaver-ER-PA10 & Panasonic Corporation, Osaka, Japan \\
\hline StepOnePlus Real-Time PCR System & ThermoFisher Scientific, Waltham, USA \\
\hline Tissue dehydration machine & Leica Biosystems, Nussloch, Germany \\
\hline Trans-Blot Turbo Transfer System & Bio-Rad Laboratories, Hercules, USA \\
\hline Tube Rotator & Stuart, Staffordshire, UK \\
\hline Ultrasound scanner Vevo 2100 & Fujifilm VisualSonics, Toronto, Candada \\
\hline Vacuum pump: BVC Control & Vacuumbrand, Wertheim, Germay \\
\hline VS120 virtual slide microscope & Olympus, Shinjuku, Japan \\
\hline Vortexer Lab Dancer & Ika-Werke, Staufen, Germany \\
\hline Vortexer Vornado & Benchmark Scientific, Edison, USA \\
\hline Water bath WNB 14 & Memmert, Schwabach, Germany \\
\hline
\end{tabular}




\subsubsection{Consumables}

Table 3. Consumables.

\begin{tabular}{|c|c|}
\hline Consumables & Company \\
\hline $\begin{array}{l}\text { Cell culture inserts }-8 \mu \mathrm{m} \text { pore size (BD } \\
353097)\end{array}$ & Sarstedt, Nümbrecht, Germany \\
\hline Cell scraper $25 \mathrm{~cm}$ & Sarstedt, Nümbrecht, Germany \\
\hline Centrifuge tube $(15 / 50 \mathrm{~mL})$ & Sarstedt, Nümbrecht, Germany \\
\hline Cryotube $1.5 \mathrm{~mL}$ & Sarstedt, Nümbrecht, Germany \\
\hline Embedding cassettes & Th. Geyer, Renningen, Germany \\
\hline Eye and nose ointment - Bepanthen & Bayer Vital, Leverkusen, Germany \\
\hline Glass coverslips $(24 \times 32,24 \times 60)$ & Menzel, Braunschweig, Germany \\
\hline Hair removal cream-Veet & RB Healthcare UK, Hull, UK \\
\hline Injection needle (sterile) & B.Braun, Melsungen, Germany \\
\hline $\begin{array}{l}\text { Insulin syringes 30G-BD Micro-Fine + } \\
\text { Demi }\end{array}$ & $\begin{array}{l}\text { Becton, Dickinson and Company, Franklin } \\
\text { Lakes, USA }\end{array}$ \\
\hline Membrane filter $0.45 \mu \mathrm{m}$ & Merck Millipore, Billerica, USA \\
\hline MicroAmp optical 96-well reaction plate & Applied Biosystems, Foster City, USA \\
\hline Microtome blade & Feather, Osaka, Japan \\
\hline Microtube $(0.5 / 1.5 / 2 \mathrm{~mL})$ & Sarstedt AG \& Co., Nuembrecht, Germany \\
\hline Nitrocellulose membrane & GE Healthcare Life Sciences, Marlborough, USA \\
\hline Optical adhesive covers & Applied Biosystems, Foster City, USA \\
\hline Parafilm & Pechiney Plastic Packaging, Menasha, USA \\
\hline PCR tubes & Sarstedt, Nümbrecht, Germany \\
\hline Scalpel & Feather, Osaka, Japan \\
\hline Serological pipette $(2 / 5 / 10 / 25 \mathrm{~mL})$ & Greiner Bio-One, Frickenhausen, Germany \\
\hline Syringe $(1 \mathrm{~mL})$ & BD Plastic, Madrid, Spain \\
\hline Syringe $(5 / 10 / 20 \mathrm{~mL})$ & B.Braun, Melsungen, Germany \\
\hline
\end{tabular}




\begin{tabular}{ll}
\hline Labsolute Sterile syringe Filter $(0.2 \mu \mathrm{m})$ & Th.Geyer Ingredients, Höxter, Germany \\
\hline TC dish 100 & Sarstedt, Nümbrecht, Germany \\
\hline TC flask (T25/75/175) & Sarstedt, Nümbrecht, Germany \\
\hline TC multiwell plates (6/12/24 wells) & Greiner Bio-One, Frickenhausen, Germany \\
\hline Thick blot filter paper & Bio-Rad, Hercules, CA, USA \\
\hline Tissue cassette & Sanowa, Leimen, Germany \\
\hline Ultrasond gel & Asmuth Medizintechnik, Minden, Germany \\
\hline Wound clips-Reflex 9 & CellPoint Scientific, Gaithersburg, USA
\end{tabular}

\subsubsection{Chemicals and reagents}

Table 4. Chemicals and reagents.

\begin{tabular}{ll}
\hline Chemical / Reagents & Company \\
\hline 6x DNA Loading Dye & Thermo Fisher Scientific, Waltham, USA \\
\hline Acrylamide solution $(30 \% / 0.8 \%)$ & AppliChem, Darmstadt, Germany \\
\hline Acetic acid $100 \%$ p.A. & AppliChem, Darmstadt, Germany \\
\hline Agarose & Nippon Genetics Europe, Düren, Germany \\
\hline Agarose protein A beads, fast flow & Merck Millipore, Billerica, USA \\
\hline Ampicillin & Carl Roth, Karlsruhe, Germany \\
\hline B-Mercaptoethanol & Merck, Darmstadt, Germany \\
\hline Bromophenol blue & Sigma-Aldrich, St. Louis, USA \\
\hline Bradford protein assay & Bio-Rad, Hercules, USA \\
\hline Bovine serum Albumin & Sigma-Aldrich, St. Louis, USA \\
\hline Chloroform p.a. & Th. Geyer, Renningen, Germany \\
\hline Citric acid monohydrate & Carl Roth, Karlsruhe, Germany \\
\hline Collagen I, rat tail & Enzo Life Sciences, Lörrach, Germany \\
\hline Dimethyl sulfoxide & Sigma-Aldrich, St. Louis, USA \\
\hline Disodium phosphate & Carl Roth, Karlsruhe, Germany \\
\hline Dithiothreitol & Sigma-Aldrich, St. Louis, USA \\
\hline & 24 \\
\hline
\end{tabular}




\begin{tabular}{ll}
\hline EDTA & Merck Millipore, Billerica, USA \\
\hline EGTA & Sigma-Aldrich, St. Louis, USA \\
\hline Eosin Y solution, aqueous & Sigma-Aldrich, St. Louis, USA \\
\hline Epidermal growth factor & Sigma-Aldrich, St. Louis, USA \\
\hline Ethanol absolute p.a. & Th. Geyer, Renningen, Germany \\
\hline Formaldehyde (37\%) & Sigma-Aldrich, St. Louis, USA \\
\hline Formaldehyde solution (4\%), buffered & Merck, Darmstadt, Germany \\
\hline FastGene 100 bp DNA marker & Nippon Genetics Europe, Düren, Germany \\
\hline GeneRuler 1 kb DNA ladder & Thermo Fisher Scientific, Waltham, USA \\
\hline Glycine & Carl Roth, Karlsruhe, Germany \\
\hline Glycerol & Carl Roth, Karlsruhe, Germany \\
\hline HEPES & Carl Roth, Karlsruhe, Germany \\
\hline Hematoxylin & Sigma-Aldrich, St. Louis, USA \\
\hline Hydrochloric acid & Carl Roth, Karlsruhe, Germany \\
\hline Hydrogen peroxide 30\% & Carl Roth, Karlsruhe, Germany \\
\hline Isopropanol & Sigma-Aldrich, St. Louis, USA \\
\hline Isoflurane & AbbVie Deutschland, Wiesbaden, Germany \\
\hline iTaq Universal SYBR Green Supermix & Bio-Rad, Hercules, CA, USA \\
\hline Immu-Mount & Thermo Fisher Scientific, Waltham, USA \\
\hline (+)-JQ1 & MedChemExpress, Monmouth Junction, USA \\
\hline Lipofectamine 2000 reagent & Invitrogen, USA \\
\hline Lithium chloride & Sigma-Aldrich, St. Louis, USA \\
\hline Matrigel & Abcam, Cambridge, UK \\
\hline Monosodium phosphate & Merck, Darmstadt, Germany \\
\hline NP-40 & Thermo Fisher Scientific, Waltham, USA \\
\hline Non-fat milk powder & Carl Roth, Karlsruhe, Germany \\
\hline Normal goat serum & Abcam, Cambridge, UK \\
\hline PageRuler Prestained Protein Ladder & Thermo Fisher Scientific, Waltham, USA \\
\hline Paraffin & Engelbrecht, Edermünde/ Besse, Germany \\
\hline & \\
\hline
\end{tabular}




\begin{tabular}{|c|c|}
\hline Paraformaldehyde & Merck, Germany \\
\hline PBS Dulbecco, powder & Biochrom, Berlin, Germany \\
\hline Phenylmethylsulfonyl fluoride & Sigma-Aldrich, St. Louis, USA \\
\hline Pierce TM ECL Western Blotting Substrate & Thermo Fisher Scientific, Waltham, USA \\
\hline Ponceau S solution & Sigma-Aldrich, St. Louis, USA \\
\hline Protease inhibitor cocktail cOmplete & Roche Diagnostics, Mannheim, Germany \\
\hline $\begin{array}{l}\text { Protein Assay Dye Reagent Concentrate } \\
\text { (Bradford reagent) }\end{array}$ & Bio-Rad, Hercules, USA \\
\hline Rotimount & Carl Roth, Germany \\
\hline Roticlear & Carl Roth, Karlsruhe, Germany \\
\hline $\begin{array}{l}\text { RotiPhenol/chloroform/isoamylalcohol } \\
(25: 24: 1)\end{array}$ & Carl Roth, Karlsruhe, Germany \\
\hline Saline $(0.9 \% \mathrm{NaCl})$ & B.Braun, Melsungen, Germany \\
\hline siLentFect Lipid reagent & Bio-Rad, Hercules, CA, USA \\
\hline Sodium chloride & Merck, Darmstadt, Germany \\
\hline Sodium dodecyl sulfate & Carl Roth, Karlsruhe, Germany \\
\hline Sodium deoxycholate & Sigma-Aldrich, St. Louis, USA \\
\hline Sodium fluoride & Sigma-Aldrich, St. Louis, USA \\
\hline Sodium orthovanadate & Sigma-Aldrich, St. Louis, USA \\
\hline Trans-Blot Turbo 5x transfer buffer & $\begin{array}{l}\text { Bio-Rad Laboratories GmbH, Neuberg, } \\
\text { Germany }\end{array}$ \\
\hline Tetrasodium pyrophosphate & Sigma-Aldrich, St. Louis, USA \\
\hline Tris-HCl & Carl Roth, Karlsruhe, Germany \\
\hline Tris-base & Carl Roth, Karlsruhe, Germany \\
\hline Triton X-100 & Carl Roth, Karlsruhe, Germany \\
\hline TRIzol & ThermoFisher Scientific, Waltham, USA \\
\hline Tumor Necrosis Factor- $\alpha$ human & Sigma-Aldrich, St. Louis, USA \\
\hline Tumor Necrosis Factor- $\alpha$ human & PeproTech, Hamburg, Germany \\
\hline Tween-20 & Sigma-Aldrich, St. Louis, USA \\
\hline Western Lightning ECL plus & PerkinElmer, Waltham, USA \\
\hline Western Lightning ECL ultra & PerkinElmer, Waltham, USA \\
\hline
\end{tabular}




Xylene AppliChem, Darmstadt, Germany

\subsubsection{Kits and plasmids}

\section{Table 5. Kits used in the study.}

\begin{tabular}{|c|c|}
\hline Kits & Company \\
\hline Bioanalyzer High Sensitivity DNA Analysis Kit & $\begin{array}{l}\text { Agilent Technologies, Santa Clara, } \\
\text { California, USA }\end{array}$ \\
\hline $\begin{array}{l}\text { CellTiter-Glo Luminescent Cell Viability Assay } \\
\text { Kit }\end{array}$ & Promega, Madison, USA \\
\hline ChIP DNA Clean \& Concentrator kit & Zymo Research Europe, Freiburg, Germany \\
\hline Human Tumor Dissociation Kit & Miltenyi Biotec, Bergisch Gladbach, Germany \\
\hline Human CCL2/MCP-1 Quantikine ELISA Kit & R\&D Systems, Minneapolis, USA \\
\hline Human TNFa Quantikine ELISA Kit & R\&D Systems, Minneapolis, USA \\
\hline iScript cDNA Synthesis Kit & Bio-Rad Laboratories, Hercules, USA \\
\hline Mouse Cell Depletion Kit & Miltenyi Biotec, Bergisch Gladbach, Germany \\
\hline Mouse TNFa Quantikine ELISA Kit & R\&D Systems, Minneapolis, USA \\
\hline Proteome Profiler Human Cytokine Array Kit & R\&D Systems, Minneapolis, USA \\
\hline $\begin{array}{l}\text { Proteome Profiler Mouse Cytokine Array Kit, } \\
\text { Panel A }\end{array}$ & R\&D Systems, Minneapolis, USA \\
\hline TruSeq RNA Library Prep Kit v2 & Illumina, San Diego, USA \\
\hline
\end{tabular}

Table 6. Plasmids used in the study.

\begin{tabular}{ll}
\hline Plasmids & Company \\
\hline pMD2.G & Addgene, Watertown, USA \\
\hline psPAX2 & Addgene, Watertown, USA \\
\hline pCDH-TNFa & Addgene, Watertown, USA \\
\hline pLKO.1 & Addgene, Watertown, USA \\
\hline pMSCV-cJUN & Addgene, Watertown, USA \\
\hline
\end{tabular}




$\begin{array}{ll}\text { pMSCV } & \text { Addgene, Watertown, USA }\end{array}$

\subsubsection{Drugs}

Table 7. Therapeutic drugs.

\begin{tabular}{ll}
\hline Drug & Company \\
\hline Carprieve & Norbrook Laboratories, Newry, UK \\
\hline Gemcitabine hydrochloride & Sigma-Aldrich, St. Louis, USA \\
\hline Oxaliplatin & Sigma-Aldrich, St. Louis, USA \\
\hline SN38 & Sigma-Aldrich, St. Louis, USA \\
\hline Buprenorphine & Bayer Vital, Leverkusen, Germany
\end{tabular}

\subsubsection{Nucleic acids}

\subsubsection{1 siRNA oligonucleotides}

Table 8. siRNA used in the study.

\begin{tabular}{lll}
\hline siRNAs & Catalogue number & Company \\
\hline Negative control siRNA & AM4611 & Thermo Fisher Scientific, Waltham, USA \\
\hline cJUN \#1 & AM16704 & Thermo Fisher Scientific, Waltham, USA \\
\hline cJUN \#2 & AM16708 & Thermo Fisher Scientific, Waltham, USA \\
\hline BRD4 \#1 & J-004937-06-0002 & Dharmacon, Lafayette, USA \\
\hline BRD4 \#2 & J-004937-07-0002 & Dharmacon, Lafayette, USA \\
\hline
\end{tabular}

\subsubsection{Primers}

Table 9. Primers used for qRT-PCR (human).

\begin{tabular}{lll}
\hline Gene & Forward primer & Reverse primer \\
\hline XS13 & TGGGACAGAACACCATGATG & AGTTTCTCCAGAGCTGGGTTGT \\
\hline
\end{tabular}




\begin{tabular}{lll}
\hline CJUN & TCCAAGTGCCGAAAAAGGAAG & CGAGTTCTGAGCTTTCAAGGT \\
\hline CCL2 & CAGCCAGATGCAATCAATGCC & TGGAATCCTGAACCCACTTCT
\end{tabular}

Table 10. Primers used for ChIP-qPCR (human).

\begin{tabular}{lll}
\hline Gene & Forward primer & Reverse primer \\
\hline CCL2 Promoter & GCTTAATGGCACCCCATCCT & GGGTCAGGACGAGACACTTTT \\
\hline CCL2 Enhancer & CCACTCACTTCTCTCACGCC & CTGTCTGCCTCCCACTTCTG \\
\hline JUN Enhancer 1 & TGCTGCTGTAGTGCACATTCT & AGCACAGACCTTTCTGCTGG \\
\hline JUN Enhancer 2 & ATGGACACTCATACATCAGAGAGC & CTCAGCGTTCTCATCCGTGT \\
\hline JUN Enhancer 3 & AGATTTCTGGGATCCGGCTTG & CCGAGGAAAGCTCTTCGCAA \\
\hline CDH1 Promoter & GGTGAACCCTCAGCCAATCAG & AGTTCCGACGCCACTGAGA \\
\hline CDH1 Enhancer 1 & GAGGCGGTATAGCCAGTTCC & CTGCTCCTAGAGGCTCCTGA \\
\hline CDH1 Enhancer 2 & GCTCGTGGAGGTTGTGTAGAA & CAAGCAGAAGCTGACAAGTTCAA \\
\hline VIM Promoter & ATCTGGGAGGCCCACGTAT & TCTTTGCTCGAATGTGCGGA \\
\hline VIM Enhancer 1 & ATTCCAAACCCCTGGATGATGTC & GGATATGCTAGTGCTCTGACTGTT \\
\hline VIM Enhancer 2 & AAGTTGCCTAAGTAACATCAATGCC & GGGTCTGAAACCCAACACACT
\end{tabular}

\subsubsection{Antibodies and enzymes}

Table 11. Antibodies used for western blot.

\begin{tabular}{llll}
\hline Antibodies & Dilution & $\begin{array}{l}\text { Catalogue } \\
\text { number }\end{array}$ & Company \\
\hline BRD4 & $1: 1000$ & ab128874 & Abcam, Cambridge, UK \\
\hline CCL2 & $1: 1000$ & MA5-17040 & Invitrogen, Eugene, USA \\
\hline cJUN & $1: 1000$ & $9165 S$ & Cell Signaling Technology, Danvers, USA \\
\hline E-Cadherin & $1: 1000$ & 610181 & BD Bioscience, San Jose, USA \\
\hline JUNB & $1: 1000$ & $3753 S$ & Cell Signaling Technology, Danvers, USA \\
\hline JUND & $1: 1000$ & sc-74 & Santa Cruz Biotechnology, Dallas, USA \\
\hline$\beta$-actin & $1: 40000$ & A3854 & Sigma-Aldrich, St. Louis, USA \\
\hline
\end{tabular}




\begin{tabular}{lccc}
\hline $\begin{array}{l}\text { Anti-mouse } \\
\operatorname{lgG}(\mathrm{HRP})\end{array}$ & $1: 6000$ & $7076 \mathrm{~S}$ & Cell Signaling Technology, Danvers, USA \\
\hline $\begin{array}{l}\text { Anti-rabbit IgG } \\
(\mathrm{HRP})\end{array}$ & $1: 6000$ & $7074 S$ & Cell Signaling Technology, Danvers, USA \\
\hline
\end{tabular}

Table 12. Antibodies used for flow cytometry analysis.

\begin{tabular}{llll}
\hline Antibodies & Dilution & Catalogue number & Company \\
\hline TNFa & $1: 20$ & 502908 & BD Bioscience, San Jose, USA \\
\hline IgG1, k isotype control & $1: 20$ & 553973 & BD Bioscience, San Jose, USA \\
\hline
\end{tabular}

Table 13. Antibodies used for immunofluorescence staining.

\begin{tabular}{llll}
\hline Antibodies & Dilution & $\begin{array}{l}\text { Catalogue } \\
\text { number }\end{array}$ & Company \\
\hline a-Amylase & $1: 100$ & 3796 & Cell Signaling Technology, Danvers, USA \\
\hline CCL2 & $1: 1000$ & MA5-17040 & Invitrogen, Eugene, USA \\
\hline CK19 & $1: 100$ & ab52625 & Abcam, Cambridge, UK \\
\hline CD45 & $1: 50$ & 550539 & BD Biosciences, San Jose, USA \\
\hline CD68 & $1: 50$ & ab955 & Abcam, Cambridge, UK \\
\hline cJUN & $1: 100$ & $9165 S$ & Cell Signaling Technology, Danvers, USA \\
\hline JUNB & $1: 100$ & $3753 S$ & Cell Signaling Technology, Danvers, USA \\
\hline KRT81 & $1: 100$ & sc-100929 & Santa Cruz Biotechnology, Dallas, USA \\
\hline TNFa & $1: 50$ & ab1793 & Abcam, Cambridge, UK \\
\hline $\begin{array}{l}\text { Goat anti-rabbit IgG- } \\
\text { Alexa Fluor 488 }\end{array}$ & $1: 500$ & A-11008 & Thermo Fisher Scientific, Waltham, USA \\
\hline $\begin{array}{l}\text { Goat anti-mouse } \\
\text { IgG-Alexa Fluor 568 }\end{array}$ & $1: 500$ & A-21124 & Thermo Fisher Scientific, Waltham, USA \\
\hline $\begin{array}{l}\text { Goat anti-rat IgG- } \\
\text { Alexa Fluor 647 }\end{array}$ & $1: 500$ & A-21247 & Thermo Fisher Scientific, Waltham, USA \\
\hline
\end{tabular}


Table 14. Antibodies used for ChIP.

\begin{tabular}{lll}
\hline Antibodies & Catalogue number & Company \\
\hline BRD4 antibody & C15410337 & Diagenode, Liege, Belgium \\
\hline cJUN antibody & $9165 S$ & Cell Signaling Technology, Danvers, USA \\
\hline JUNB antibody & $3753 S$ & Cell Signaling Technology, Danvers, USA \\
\hline H3K27ac antibody & GTX128944 & GeneTex, Alton Pkwy Irvine, USA \\
\hline Rabbit IgG & C15410206 & Diagenode, Liege, Belgium \\
\hline
\end{tabular}

Table 15. Enzymes used in the study.

\begin{tabular}{lll}
\hline Enzymes & Catalogue number & Company \\
\hline Proteinase K & A4392,0005 & AppliChem, Darmstadt, Germany \\
\hline $\begin{array}{l}\text { SuperScript II Reverse } \\
\text { Transcriptase }\end{array}$ & 18064022 & Thermo Fisher Scientific, Waltham, USA \\
\hline RNase A & R6513 & Sigma-Aldrich, St. Louis, USA \\
\hline
\end{tabular}

\subsubsection{Buffers}

\subsubsection{Buffers for Western blot}

Table 16. Whole cell lysates (WCL) buffer.

\begin{tabular}{ccc} 
Name & Components & Final concentration \\
\hline HEPES $(\mathrm{pH} 7.5)$ & $50 \mathrm{mM}$ \\
$\mathrm{NaCl}$ & $150 \mathrm{mM}$ \\
\hline WCL buffer & EGTA & $1 \mathrm{mM}$ \\
\hline Glycine & $10 \%(\mathrm{v} / \mathrm{v})$ \\
\hline Triton X-100 & $1 \%(\mathrm{v} / \mathrm{v})$ \\
$\mathrm{NaF}$ & $100 \mathrm{mM}$ \\
\hline $\begin{array}{c}\text { Tetrasodium } \\
\text { pyrophosphate }\end{array}$ & $10 \mathrm{mM}$ \\
\hline
\end{tabular}


Table 17. Laemmli buffer (5x).

\begin{tabular}{ccc}
\hline Name & Components & Final concentration \\
\hline \multirow{3}{*}{ Laemmli buffer } & $225 \mathrm{mM}$ \\
\cline { 2 - 3 } & Glycerin $(\mathrm{pH} 6.8)$ & $50 \%(\mathrm{v} / \mathrm{v})$ \\
\hline SDS & $5 \%(\mathrm{v} / \mathrm{v})$ \\
\hline DTT & $100 \mathrm{mM}$ \\
\hline B-Mercaptoethanol & $0.02 \%(\mathrm{v} / \mathrm{v})$ \\
\hline
\end{tabular}

Table 18. Separating gel buffer and stacking gel buffer stocks.

\begin{tabular}{ccc}
\hline Name & Components & Final concentration \\
\hline $\begin{array}{c}\text { Separating gel buffer stock } \\
(\mathrm{pH} \text { 8.8) }\end{array}$ & Tris-base & $1.5 \mathrm{M}$ \\
\cline { 2 - 3 } & & $0.4 \%(\mathrm{v} / \mathrm{v})$ \\
\hline $\begin{array}{c}\text { Stacking gel buffer stock } \\
(\mathrm{pH} \mathrm{6.8)}\end{array}$ & SDS & $0.5 \mathrm{M}$ \\
\cline { 2 - 3 } & Tris-base & $0.4 \%(\mathrm{v} / \mathrm{v})$ \\
\end{tabular}

Table 19. Separating and stacking buffer working solution.

\begin{tabular}{ccc}
\hline Name & Components & Volume \\
& Separating gel buffer stock & $20 \mathrm{~mL}$ \\
\cline { 2 - 3 } $\begin{array}{c}\text { Separating gel buffer } \\
10 \%\end{array}$ & $\begin{array}{c}\text { Acrylamide solution } \\
(30 \% / 0.8 \%)\end{array}$ & $26.6 \mathrm{~mL}$ \\
\cline { 2 - 3 } & Glycerol & $4 \mathrm{~mL}$ \\
\cline { 2 - 3 } Separating gel buffer & Aqua dest & $29.3 \mathrm{~mL}$ \\
\cline { 2 - 3 } & Separating gel buffer stock & $20 \mathrm{~mL}$ \\
& Acrylamide solution \\
& $(30 \% / 0.8 \%)$ & $40 \mathrm{~mL}$ \\
\end{tabular}


$15 \%$

Glycerol

$4 \mathrm{~mL}$

Aqua dest

$16 \mathrm{~mL}$

Table 20. Running buffer (10x).

\begin{tabular}{ccc}
\hline Name & Components & Final concentration \\
\hline \multirow{3}{*}{ Running buffer } & Tris-base & $250 \mathrm{mM}$ \\
& Glycerin & $1.92 \mathrm{M}$ \\
\cline { 2 - 3 } & SDS & $1 \%(\mathrm{w} / \mathrm{v})$ \\
\hline
\end{tabular}

Table 21. Transfer buffer (1L).

\begin{tabular}{ccc} 
Name & Components & Final concentr \\
\multirow{3}{*}{ Transfer buffer } & $5 x$ Transfer buffer & $200 \mathrm{~mL}$ \\
& Ethanol & $200 \mathrm{~mL}$ \\
\cline { 2 - 3 } & Aqua dest & $600 \mathrm{~mL}$
\end{tabular}

Table 22. TBS buffer (10x).

\begin{tabular}{ccc}
\hline Name & Components & Final concentration \\
\hline TBS buffer & Tris-base & $121.4 \mathrm{~g}$ \\
$(\mathrm{pH} 7.6)$ & $\mathrm{NaCl}$ & $400.31 \mathrm{~g}$ \\
\cline { 2 - 3 } & Aqua dest & $5 \mathrm{~L}$ \\
\hline
\end{tabular}

Table 23. TBST buffer (10x).

\begin{tabular}{ccc}
\hline Name & Components & Final concentration \\
\hline TBST buffer & TBS & $1 \mathrm{X}$ \\
& Tween & $0.1 \%(\mathrm{w} / \mathrm{v})$ \\
\hline
\end{tabular}


Table 24. Blocking solution.

\begin{tabular}{ccc}
\hline Name & Components & Final concentration \\
\hline Blocking solution & TBST & $1 \mathrm{X}$ \\
& Non-fat milk & $5 \%(\mathrm{w} / \mathrm{v})$ \\
\cline { 2 - 3 } & & 5
\end{tabular}

\subsubsection{Buffers for immunofluorescence staining}

Table 25. Citrate buffer.

\begin{tabular}{ccc}
\hline Name & Components & Final concentration \\
\hline Citrate buffer & Citric acid monohydrate & $2.1 \mathrm{~g}$ \\
\cline { 2 - 3 }$(\mathrm{pH} \mathrm{6.0)}$ & Aqua dest & $1 \mathrm{~L}$ \\
\hline
\end{tabular}

Table 26. PB buffer.

\begin{tabular}{ccc}
\hline Name & Components & Final concentration \\
\hline PB buffer & Monosodium phosphate & $2.4 \mathrm{~g}$ \\
\cline { 2 - 3 }$(\mathrm{pH} 7.4)$ & Disodium phosphate & $11.31 \mathrm{~g}$ \\
\cline { 2 - 3 } & Aqua dest & $1 \mathrm{~L}$ \\
\hline
\end{tabular}

Table 27. PBT buffer.

\begin{tabular}{ccc}
\hline Name & Components & Final concentration \\
\hline PBT buffer & PB & $1 \mathrm{X}$ \\
& Triton X-100 & $0.4 \%(\mathrm{v} / \mathrm{v})$ \\
\hline
\end{tabular}




\subsubsection{Buffers for Chromatin Immuno-Precipitation}

Table 28. Nelson buffer.

\begin{tabular}{ccc}
\hline Name & Components & Final concentration \\
\hline \multirow{2}{*}{ Nelson buffer } & $\mathrm{NaCl}$ & $150 \mathrm{mM}$ \\
\cline { 2 - 3 } & EDTA $(\mathrm{pH} \mathrm{8)}$ & $20 \mathrm{mM}$ \\
\hline Tris- $\mathrm{HCl}(\mathrm{pH} 7.5)$ & $50 \mathrm{mM}$ \\
$\mathrm{NP}-40$ & $0.5 \%$ \\
\hline Triton X-100 & $1 \%$ \\
\hline $\mathrm{NaF}$ & $20 \mathrm{mM}$
\end{tabular}

Table 29. Gomes wash buffer.

\begin{tabular}{|c|c|c|}
\hline Name & Components & Final concentration \\
\hline \multirow{6}{*}{ Gomes wash buffer } & Tris-HCl (pH 8.5) & $100 \mathrm{mM}$ \\
\hline & $\mathrm{LiCl}$ & $500 \mathrm{mM}$ \\
\hline & NP-40 & $1 \%(v / v)$ \\
\hline & Sodium deoxycholate & $1 \%(w / v)$ \\
\hline & $\mathrm{NaF}$ & $20 \mathrm{mM}$ \\
\hline & EDTA & $20 \mathrm{mM}$ \\
\hline
\end{tabular}

Table 30. Weinmann lysis buffer.

\begin{tabular}{ccc}
\hline Name & Components & Final concentration \\
\hline \multirow{3}{*}{ Weinmann lysis buffer } & Tris- $\mathrm{HCl}(\mathrm{pH} \mathrm{8.0)}$ & $50 \mathrm{mM}$ \\
\cline { 2 - 3 } & EDTA & $10 \mathrm{mM}$ \\
\cline { 2 - 3 } & SDS & $1 \%(\mathrm{v} / \mathrm{v})$
\end{tabular}




\subsubsection{Cell culture}

Table 31. Cell culture components.

\begin{tabular}{ll}
\hline Components & Company \\
\hline Bovine pituitary extract & Sigma-Aldrich, St. Louis, USA \\
\hline Defined Keratinocyte-SFM Medium & ThermoFisher Scientific, Waltham, USA \\
\hline Dulbecco's Modified Eagle Medium (DMEM) & ThermoFisher Scientific, Waltham, USA \\
\hline Fetal calf serum (FCS) & BioWest, Nuaillé, France \\
\hline Non-essential amino acids (NEAA) & ThermoFisher Scientific, Waltham, USA \\
\hline Opti-MEM & ThermoFisher Scientific, Waltham, USA \\
\hline PBS (-Ca/-Mg) & ThermoFisher Scientific, Waltham, USA \\
\hline Penicillin-streptomycin solution & Sigma-Aldrich, St. Louis, USA \\
\hline Roswell Park Memorial Institute (RPMI) & ThermoFisher Scientific, Waltham, USA \\
1640 Medium & \\
\hline Polybrene & Santa Cruz Biotechnology, Dallas, USA \\
\hline Puromycin & Sigma-Aldrich, St. Louis, USA \\
\hline
\end{tabular}

\subsection{Methods}

\subsubsection{Animal studies}

Animal experiments conducted in this study were approved by the University of Göttingen Central Animal-experimental Facility.

Kras ${ }^{G 12 D}$; Trp53 ${ }^{R 172 H} ; P d x C r e(K P C)$ tumor tissues used in this study were provided by D. Spyropoulou (Department of Gastroenterology, GI Oncology \& Endocrinology, UMG, Germany). In this model, Kras and Trp53 were both mutated specifically in the pancreas to induce PDAC as described previously (Hingorani et al., 2005). Histopathological classification of KPC tumors into well-to-moderately (W/M) (G1/G2), or poorly differentiated (G3/G4), was performed by expert pathologist P. Ströbel (Department of Pathology, UMG, Germany). 


\subsubsection{Orthotopic mouse models}

10-week old male NMRI-Foxn1nu/nu mice were used to generate orthotopic PDAC mouse models. Mice were injected with buprenorphine $(0.1 \mathrm{mg} / \mathrm{kg}$ body weight) intraperitoneally (i.p.) 30 mins before transplantation. Mice were then kept on a warm plate and anesthetized by inhalation of 2.5 $-3.5 \%$ isoflurane in oxygen for surgery. Mice were subcutaneously injected with Carprieve (Norbrook Laboratories, Newry, UK; 5 mg/kg body weight) to ease the pain and inflammation immediately before surgery and one to two days after surgery if necessary. After confirming there was no stimuli reflection, skin was opened ( $\sim \mathrm{cm}$ cut) on the left side of the mice where pancreas resides. $1 \times 10^{6}$ PDAC cells (CAPAN1, CAPAN2, MiaPaCa2, and PANC1) were suspended in $15 \mu \mathrm{L}$ culture medium and injected into the tail of the pancreas with an insulin syringe.

For establishing the syngeneic KPC orthotopic mouse model, 10-week old male C57BL6J mice were anesthetized for hair removal before surgery, as described previously (Patzak et al., 2019). $3.5 \times 10^{4} \mathrm{KPC}$ cells were injected into the tail of the pancreas. The rest of the procedures was carried out as above.

\subsubsection{Sonography}

Three weeks post transplantation, small animal high-resolution ultrasound was applied weekly to determine the tumor growth, as described previously (Patzak et al., 2019). Briefly, mice were put under isoflurane anesthesia and fixed with surgical tape so that each leg was restrained. Ultrasound gel was applied on the ventral body surface. Subsequently, ultrasound imaging was carried out using an Ultrasound scanner Vevo 2100 (Fujifilm VisualSonics, Toronto, Canada).

\subsubsection{Treatment}

For TNFa studies, CAPAN1-transplanted mice were randomized into two groups. TNFa (PeproTech $\mathrm{GmbH}$, Hamburg, Germany; $0.4 \mathrm{mg} / \mathrm{kg}$ body weight) was administrated via i.p. injection three times a week for in total three weeks. Aqua dest. was administrated in control mice. 
For anti-TNFa studies, mice were randomized before treatment and administrated with anti-TNF $\alpha$ antibody (Hölzel Biotech, Köln, Germany; $10 \mu \mathrm{g} / \mathrm{g}$ body weight) or lgG isotype control (Hölzel Biotech, Köln, Germany; $10 \mu \mathrm{g} / \mathrm{g}$ body weight) three times a week after 10 days of transplantation.

For JQ1 studies, mice were randomized before treatment. 10 days after transplantation, JQ1 (MedChemExpress, Monmouth Junction, USA; $50 \mathrm{mg} / \mathrm{kg}$ body weight,) was administrated via i.p injection daily for three weeks. DMSO was administrated in control mice.

\subsubsection{Tissue harvesting}

Mice were euthanized by inhalation of $\mathrm{CO}_{2}$ and subsequent cervical dislocation. Whole pancreas, spleen, liver, and blood samples were collected. Samples of pancreatic tumor tissue were snap-frozen and stored at $-80^{\circ} \mathrm{C}$ for further analysis. The rest of tissues were fixed in $4 \%$ formaldehyde solution overnight. Blood samples in heparin tubes were centrifuged at $4000 \mathrm{Xg}$ for $10 \mathrm{~min}$ at room temperature. Serum was transferred to a microtube and stored at $-80 \stackrel{\circ}{\circ}$.

\subsubsection{Paraffin embedding and tissue sectioning}

Formaldehyde-fixed tissues were processed with an automatic tissue processer (Leica Biosystems, Nussloch, Germany) prior to paraffin embedding. The dehydration protocol is as follow: 75 min formaldehyde solution, 30 min $55 \%$ ethanol, 45 min $85 \%$ ethanol, 60 min $96 \%$ ethanol, 75 min $99 \%$ ethanol, 70 min $99 \%$ ethanol, 90 min $99 \%$ ethanol, 20 min xylene, 30 min xylene, 70 min xylene, 30 min paraffin, 45 min paraffin, $90 \mathrm{~min}$ paraffin. Tissues were then embedded in paraffin blocks. A microtome was used to section tissues at a thickness of $4 \mu \mathrm{m}$. Sections were kept at $37^{\circ} \mathrm{C}$ overnight before further staining.

\subsubsection{Hematoxylin and Eosin Staining}

Tissue sections were deparaffinized in xylene (twice for $30 \mathrm{~min}$ ). After rehydration in and ethanol series (99\%, $95 \%, 70 \%$, each $1 \mathrm{~min}$ ), slides were kept in aqua dest. for 3 min. 
Subsequently, slides were incubated in hematoxylin solution for 8 min followed by a washing step in running tap water for $7 \mathrm{~min}$. Slides were then transferred shortly (5 s) in aqua dest. containing $0.2 \%$ acetic acid followed by incubation in eosin solution (in $0.2 \%$ acetic acid) for $15 \mathrm{~s}$. Next, slides were dehydrated in increasing concentrations of ethanol ( $70 \%$ for 30 s, $95 \%$ for 1.5 min, $100 \%$ for 2 min). Finally, slides were cleared in Roticlear and fixed with Rotimount.

\subsubsection{Tile image}

Whole tile image scans of H\&E and IF staining were carried out using a VS120 virtual slide microscope (Olympus, Shinjuku, Japan), and resulting images were evaluated using cellSens Dimension software (Olympus, Shinjuku, Japan).

\subsubsection{Immunofluorescence (IF) staining and quantification}

IF was carried out as previously described (Singh et al., 2016). After deparaffinization with two times washing of xylene for 30 mins, sections were rehydrated in a descending order of ethanol ( $99 \%, 96 \%, 80 \%$, each 4 min). Antigen retrieval was achieved by keeping slides boiling in citrate buffer $(\mathrm{pH} \mathrm{6.0)}$ for 6 minutes in a microwave, then cooling down for $10 \mathrm{~min}$ at room temperature. Subsequently, sections were kept on ice for $20 \mathrm{~min}$ and washed five times for 5 min with PB. Afterwards, sections were blocked in $10 \%$ normal goat serum (NGS; Abcam, Cambridge, UK) diluted in PBT buffer for $1.5 \mathrm{~h}$ at $4^{\circ} \mathrm{C}$. Primary antibodies (Table 13) were diluted as indicated in PBT buffer containing $2 \%$ NGS and added onto each slide (100-200 $\mu \mathrm{L})$, keeping in a humid box overnight at $4^{\circ} \mathrm{C}$. Next day, sections were washed five times for 5 min each with PB and subjected to secondary antibodies staining (Table 13) for $2 \mathrm{~h}$ at $4^{\circ} \mathrm{C}$. Secondary antibodies were diluted in PBT buffer containing $2 \% \mathrm{NGS}$. DAPI was diluted to $20 \mu \mathrm{g} / \mathrm{mL}$ in PB and added to sections for $10 \mathrm{~min}$ at room temperature. In the end, slides were fixed in Immu-Mount (Thermo Fisher Scientific, Waltham, MA, USA) with a coverslip. IF images were acquired via FluoView 1000 confocal microscopy (Olympus FluoView 1000) and quantified either manually by counting positive cells or by measuring staining intensity (as integrated density above a set threshold, given as arbitrary units (A.U.)) using ImageJ Fiji software (Schindelin et al., 2012). 


\subsubsection{Cell culture}

\subsubsection{Cell culture for established PDAC cell lines}

Human PDAC cell lines (CAPAN1, CAPAN2, PANC1, and MiaPaCa2) were obtained from the ATCC. PT45P1 cell line was kindly provided by G. Natoli (Humanitas University, Malian, Italy). CAPAN1, CAPAN2, and PT45P1 were cultured in Roswell Park Memorial Institute (RPMI) 1640 Medium (ThermoFisher Scientific, Waltham, USA) supplemented with $10 \%$ fetal calf serum (FCS; BioWest, Nuaillé, France). PANC1 and MiaPaCa2 were cultured in Dulbecco's modified Eagle's Medium (DMEM; ThermoFisher Scientific, Waltham, USA) supplemented with $10 \%$ FCS. The primary PDAC cell line derived from Kras ${ }^{\text {G12D; }}$ Trp53 ${ }^{R 172 H}$; PdxCre (KPC) mice has been described previously (Patzak et al., 2019). KPC cells were maintained in DMEM supplemented with $10 \%$ FCS and $1 \%$ nonessential amino acids (NEAA; ThermoFisher Scientific, Waltham, USA). All cell lines were kept at $37^{\circ} \mathrm{C}$ and $5 \% \mathrm{CO}_{2}$.

For thawing cells, stocks were immediately defrosted in a $37^{\circ} \mathrm{C}$ water bath and then mixed with $8 \mathrm{~mL}$ complete culture medium, followed by centrifugation at $1200 \mathrm{rpm}$ for 3 min. The cell pellet was resuspended in $10 \mathrm{~mL}$ culture medium and transferred into a culture flask. Cells were passaged at least once after thawing before conducting any functional experiment.

For making cell stocks, cells pellets were resuspended in freezing medium [90 \% FCS plus $10 \%$ dimethyl sulfoxide (DMSO)] and aliquoted into cryotubes. Cells were stored in Mr. Frosty containers in a $-80 \stackrel{\circ}{C}$ freezer overnight and then transferred into liquid nitrogen for long term storage.

\subsubsection{Generation of primary PDAC cells from patient-derived xenografts}

The used patient-derived xenograft (PDX) model was established by S. Hahn (RuhrUniversity Comprehensive Cancer Center, Bochum, Germany). Surgically resected PDAC tissues collected from patients were implanted subcutaneously in NMRI Foxn $1^{\text {nu/nu }}$ mice. Resulting xenograft tumors were isolated and re-implanted in both flanks of mice for at least three generations. The PDX tumors were harvested and subsequently 
dissociated by gentleMACS dissociator (Miltenyi Biotec, Bergisch Gladbach, Germany). For the isolation of primary PDX tumor cells, the human tumor dissociation kit and mouse cell depletion kit (Miltenyi Biotec, Bergisch Gladbach, Germany) were utilized according to the manufacturer's protocol. Selected primary PDX tumor cell lines (GCDX5, GCDX57, and GCDX62) were cultured in type I collagen-coated dishes for several passages, and then transferred to normal culture vessels for further experiments. Xenografting and generation of PDX cell lines was performed by S.K. Singh, E. Hessmann and W. Kopp (Department of Gastroenterology, GI Oncology \& Endocrinology, UMG, Germany). The study was approved by the ethics committee of the Ruhr University Bochum (permission no. 3534-9, 3841-10, 16-5792) and the University Medical Center Göttingen (permission no. 70112108). All animal experiments were performed according to the guidelines of the local Animal Use and Care Committees at the Ruhr University Bochum (8.8750.10.32.09.018) and the Central Animal-experimental Facility at the University of Göttingen (14/1634, 15/2057, 18/2953).

GCDX cell lines were maintained in a 3:1 mixture of Keratinocyte-SFM (KSF) and RPMI media, supplemented with $2 \%(\mathrm{v} / \mathrm{v}) \mathrm{FCS}, 1 \%(\mathrm{v} / \mathrm{v})$ Penicillin-streptomycin, bovine pituitary extract (BPE), and epidermal growth factor (hEGF).

\subsubsection{Generation of lentiviral and retroviral transduced stable cell lines}

For the preparation of virion, $2 \times 10^{6}$ HEK-293T (clone 17) cells (ATCC, Manassas, Virginia, USA) were seeded in a $10 \mathrm{~cm}$ dish. The next day, HEK-293T cells were transfected with gene of interest $(\mathrm{GOI})$-containing plasmid $(10.67 \mu \mathrm{g})$, envelope vector $(5.33 \mu \mathrm{g})$ with either $8 \mu \mathrm{g}$ of lentiviral psPAX2 or retroviral pKAT (Finer et al., 1994) packaging vectors using Lipofectamine 2000 (Invitrogen, USA). The cell supernatant containing virus was collected and filtered through $0.45 \mu \mathrm{m}$ membrane filter (Merck Millipore, Billerica, USA). The virus-containing media and polybrene were then used to transduce target cells (Santa Cruz Biotechnology, Dallas, USA). $24 \mathrm{~h}$ post transduction, fresh culture medium was added to replace the virus-containing medium. After $72 \mathrm{~h}$, cells were selected by $2 \mu \mathrm{g} / \mathrm{mL}$ puromycin (Sigma-Aldrich, St. Louis, USA) and maintained subsequently in $0.5-1 \mu \mathrm{g} / \mathrm{mL}$ culture medium. All the constructs used are listed in Table 
6. All the transduced stable cell lines were kindly generated by K. Bojarczuk and B. Chapuy (Department of Hematology and Medical Oncology, UMG, Germany).

\subsubsection{Cell viability assay for chemosensitivity experiments}

The CellTiter-Glo Luminescent Cell Viability Assay (Promega, Madison, USA) was performed to quantify cell viability according to the manufacturer's instructions. For individual experiments, $6 \times 10^{3}$ cells were seeded in 96-well plates in $100 \mu \mathrm{L}$ per well 24 h prior to treatment. Gemcitabine, SN38 (an active Irinotecan metabolite) or oxaliplatin were diluted to different concentrations as indicated in the figures and applied to individual wells. After $72 \mathrm{~h}$, plates were equilibrated to RT for $30 \mathrm{~min}$. Cells were subsequently incubated with $30 \mu \mathrm{L}$ CellTiter-Glo solution and kept on a shaker (400-600 rpm) for $2 \mathrm{~min}$, and incubated in the dark for $10 \mathrm{~min}$. The mixture was then transferred into a 96 -well white plate and subjected to measurement of the luminescence via a LUmo Luminometer at an integration time of one second per well.

\subsubsection{Flow cytometry analysis}

$1 \times 10^{6}$ cells were taken freshly for the cell surface staining of TNFa or TNFa-mCherry reporter gene. Firstly, cells were transferred into a 96-well plate (round bottom) at a concentration of $1 \times 10^{5}$ cells/well. Subsequently, cells were centrifuged at $300 \mathrm{Xg}$ for 5 min and washed once with FACS buffer (10\% knock out serum in -Ca/-Mg PBS; ThermoFisher Scientific, Waltham, USA). PDAC cells were either stained with primary antibodies or untreated control for $30 \mathrm{~min}$ at RT. Cells were then washed with $200 \mu \mathrm{L}$ FACS buffer twice prior to flow cytometry analysis. For endogenous TNFa expression, $488 \mathrm{~nm}$ laser was used to detect established PDAC cell lines (CAPAN1, CAPAN2, PANC1, and MiaPaCa2). For the mCherry expression, $561 \mathrm{~nm}$ laser was used to detect TNFa-mCherry overexpressing cells (TNFa-OE). Antibodies used for this study are listed in Table 12.

\subsubsection{6 siRNA transfection}

$2.5 \times 10^{5}$ cells were seeded in 6 -well plates ( $2 \mathrm{~mL}$ per well). The next day, culture media was freshly changed to $1 \mathrm{~mL} 30 \mathrm{~min}$ prior to transfection. For each well, transfection 
mixture was prepared by adding $6 \mu \mathrm{L}$ siLentFect lipid reagent (Bio-Rad, Hercules, CA, USA) and $6 \mu \mathrm{L}$ of $20 \mu \mathrm{M}$ sicJUN, siBRD4 or non-targeting siRNA to $200 \mu \mathrm{L}$ Opti-MEM medium (ThermoFisher Scientific, Waltham, USA). The lipid-siRNA mixture was incubated at room temperature for $15 \mathrm{~min}$ and then added into wells. Cells were incubated with siRNA for $24 \mathrm{~h}$, after which the medium was exchanged. Protein or RNA was harvested 48-72 $\mathrm{h}$ after transfection. siRNA sequences used for this study are listed in Table 8.

\subsubsection{Trans-well invasion assay}

$16 \mu \mathrm{L}$ type I collagen stock was diluted in $984 \mu \mathrm{L} 0.1 \mathrm{M} \mathrm{HCl}$ in a microtube. The bottom of $8 \mu \mathrm{m}$ porous inserts for 24-well plates were coated with $50 \mu \mathrm{L}$ diluted collagen, which solidifies after 1-2 h. Matrigel (Abcam, Cambridge, UK) was thawed on ice and $50 \mu \mathrm{L}$ were mixed with $10 \mu \mathrm{L}$ medium containing $1 \times 10^{5}$ cells. The solution was seeded in coated inserts in duplicates and incubated at $37^{\circ} \mathrm{C}$ for $30 \mathrm{~min}$. Subsequently, $250 \mu \mathrm{L}$ culture medium was added to inserts and $750 \mu \mathrm{L}$ to the wells. After $48 \mathrm{~h}$, Matrigel was removed from the inserts with cotton swabs. Invaded cells on membranes were fixed with $4 \%$ PFA, followed by three times washing of PBS. The membranes of inserts were removed and stained with $20 \mu \mathrm{g} / \mathrm{mL}$ DAPI for $1 \mathrm{~min}$. Pictures were acquired using a FluoView 1000 confocal microscope and FV10-ASW software version 4.2 (Olympus, Shinjuku, Japan). The invaded cells were counted manually per 20X field of view (F.o.V) from 10 pictures per replicate.

\subsubsection{Molecular biology}

\subsubsection{RNA extraction}

Cells were washed twice with PBS, scraped in $800 \mu \mathrm{L}$ TRIzol (ThermoFisher Scientific, Waltham, USA) and collected in a $1.5 \mathrm{~mL}$ microtube. $200 \mu \mathrm{L}$ chloroform were added to each sample, followed by vortexing and centrifugation at $13000 \mathrm{Xg}$ for $15 \mathrm{~min}$ at $4{ }^{\circ} \mathrm{C}$. The aqueous phase was transferred into a new tube which allows RNA precipitation by incubation with $500 \mu \mathrm{L}$ iso-propanol at room temperature for $10 \mathrm{~min}$. Samples were then centrifuged at $13000 \mathrm{Xg}$ for $30 \mathrm{~min}$ at $4{ }^{\circ} \mathrm{C}$ and subsequently washed twice with $1 \mathrm{~mL}$ 
$75 \%$ ethanol. Dried pellets were ultimately dissolved in $30 \mu \mathrm{L}$ aqua dest. and stored at $80 \stackrel{\circ}{\circ}$.

\subsubsection{2 cDNA synthesis}

RNA concentration was measured using a Nano Photometer-P330 (Intas Science Imaging, Göttingen, Germany). $1 \mu \mathrm{g}$ RNA was used for cDNA synthesis using the iScript cDNA synthesis kit (Bio-Rad Laboratories, Hercules, USA) as per manufacturer's instructions. cDNA synthesis was performed in a thermal cycler as follows: $25{ }^{\circ} \mathrm{C}$ for 5 min, $46 \stackrel{\circ}{\mathrm{C}}$ for $20 \mathrm{~min}, 95 \stackrel{\circ}{\mathrm{C}}$ for $1 \mathrm{~min}$. The $20 \mu \mathrm{L}$ reaction volume was finally diluted with $80 \mu \mathrm{L}$ aqua dest.

\subsubsection{3 qRT-PCR}

Quantitative real-time PCR (qRT-PCR) reaction was prepared as shown in Table 32:

Table 32. qRT-PCR reaction volume.

\begin{tabular}{cc}
\hline Reactions & Volume per single $\mathbf{1 0} \mu \mathrm{L}$ reaction \\
\hline SYBR green mix & $5 \mu \mathrm{L}$ \\
\hline Aqua dest. & $3.5 \mu \mathrm{L}$ \\
\hline Forward primer & $0.25 \mu \mathrm{L}$ \\
\hline Reverse primer & $0.25 \mu \mathrm{L}$ \\
\hline CDNA & $1 \mu \mathrm{L}$ \\
\hline
\end{tabular}

qRT-PCR primers used in this study are listed in Table 9. All samples were pipetted in triplicates and performed by StepOnePlus Real-Time PCR System (Applied Biosystems, Foster City, USA) with the program shown in Table 33:

Table 33. qRT-PCR program.

\begin{tabular}{cccc}
\hline Program & Temperature & Time & Cycle number \\
\hline Initiating phase & $95^{\circ} \mathrm{C}$ & $10 \mathrm{~min}$ & \\
\hline $95^{\circ} \mathrm{C}$ & $15 \mathrm{~s}$ & $\begin{array}{c}40 \text { cycles (mRNA } \\
\text { qPCR) or 55 cycles } \\
\text { (ChIP-qPCR) }\end{array}$ \\
\hline $60^{\circ} \mathrm{C}$ & $1 \mathrm{~min}$ & \\
\hline
\end{tabular}




\begin{tabular}{llc} 
& $95^{\circ} \mathrm{C}$ & $15 \mathrm{~s}$ \\
\cline { 2 - 3 } Melting curve & $60^{\circ} \mathrm{C}$ & $1 \mathrm{~min}$ \\
\cline { 2 - 3 } & $95^{\circ} \mathrm{C}$ & $15 \mathrm{~s}$
\end{tabular}

\subsubsection{Chromatin immunoprecipitation}

Cells were cultured in $15 \mathrm{~cm}$ culture dishes until they reached $80-90 \%$ confluency and then fixed with $1 \%$ formaldehyde at room temperature for 15-20 min. 1.25 M glycine (Carl Roth, Karlsruhe, Germany) was added for $5 \mathrm{~min}$ to quench the reaction. Nelson buffer containing protease and phosphatase inhibitors (1 mM PMSF, $1 \mathrm{mM} \mathrm{NaO}, 10 \mathrm{mM} \mathrm{NaF}$, and $1 \mathrm{X}$ cocktail cOmplete) was used to lyse the cells to release nuclei. After centrifugation at $12000 \mathrm{Xg}$ for 2 min at $4{ }^{\circ} \mathrm{C}$, nuclei pellet was suspended in Gomes lysis buffer containing $0.1 \%$ SDS, protease and phosphatase inhibitors (1 mM PMSF, $1 \mathrm{mM}$ $\mathrm{NaO}, 10 \mathrm{mM} \mathrm{NaF}$, and $1 \mathrm{X}$ cocktail cOmplete). The suspension was aliquoted into sonication tubes as $250 \mu \mathrm{L}$ per tube prior to sonication, which was performed by Diagenode Biorupter-Pico with 30 seconds ON/OFF pulse for 10-15 cycles. $30 \mu \mathrm{L}$ sheared chromatin was incubated with RNase A $\left(0.75 \mu \mathrm{L}\right.$ from $30 \mathrm{mg} / \mathrm{ml}$ stock; $55^{\circ} \mathrm{C}, 30$ min; Sigma-Aldrich, St. Louis, USA) and proteinase $\mathrm{K}\left(1 \mu \mathrm{L}\right.$ from $20 \mathrm{mg} / \mathrm{mL}$ stock; $65^{\circ} \mathrm{C}$, overnight; AppliChem, Darmstadt, Germany) and then loaded on a $1.5 \%$ agarose gel for the confirmation of fragment size. Properly sheared chromatin (from 200 to $600 \mathrm{bp}$ ) was then pre-cleared by incubation with $100 \mu \mathrm{L} 50 \%$ slurry protein $\mathrm{A}$ agarose beads on a rotator for $1 \mathrm{~h}$ at $4{ }^{\circ} \mathrm{C}$. Subsequently, samples were centrifuged at $12000 \mathrm{Xg}$ for $2 \mathrm{~min}$ at $4 \stackrel{\circ}{ } \mathrm{C}$ and the supernatant aliquoted for the gene of interest, IgG isotype control, and input. If not processed immediately, samples were snap-frozen and kept at $-80{ }^{\circ} \mathrm{C}$. ChIP-grade primary antibodies were incubated with samples on rotator overnight at $4 \stackrel{\circ}{\circ} \mathrm{C}$. Agarose beads were blocked in BSA $(1 \mu \mathrm{g} / \mu \mathrm{L})$ diluted in Gomes lysis buffer. The next day, the protein-antibody complex solution was incubated with blocked beads for $2 \mathrm{~h}$ at $4 \stackrel{\circ}{\circ} \mathrm{C}$. Immune complex was washed as follow: 1 time Gomes lysis buffer, 2 times Gomes wash buffer, 2 times Gomes lysis buffer, and 2 times TE buffer to remove unspecific binding. Washed chromatin was then incubated with RNase A $\left(0.2 \mu \mathrm{g} / \mu \mathrm{L} ; 55^{\circ} \mathrm{C}, 30 \mathrm{~min}\right.$; SigmaAldrich, St. Louis, USA) and proteinase $\mathrm{K}\left(1 \mu \mathrm{L}\right.$ from $20 \mathrm{mg} / \mathrm{mL}$ stock; $65^{\circ} \mathrm{C}$, overnight; 
AppliChem, Darmstadt, Germany) prior to DNA extraction. DNA was extracted by phenol/chloroform/isoamyl alcohol solution and washed once with $70 \%$ ethanol. Final DNA was resuspended in 20-30 $\mu \mathrm{L}$ aqua dest. and stored at $-20 \stackrel{\circ}{ } \mathrm{C}$ for further analysis.

\subsubsection{Protein sample preparation}

For the preparation of whole cell lysates, cells were washed twice with ice-cold PBS and lysed in WCL buffer on ice for $30 \mathrm{~min}$. Lysates were then centrifuged at $12000 \mathrm{X} \mathrm{g}$ for 20 min and supernatant was collected for Western blot analysis.

\subsubsection{Bradford assay}

Protein concentration/content was determined using Bradford reagent (Bio-Rad, Hercules, CA, USA). Briefly, BSA was diluted to different concentrations for the establishment of a standard curve. $1 \mu \mathrm{L}$ of each protein sample was added to $200 \mu \mathrm{L}$ Bradford reagent. The absorbance value was measured by PHOMO plate reader at $595 \mathrm{~nm}$. The absorbance of samples was then used to calculate the protein concentration using the standard curve.

\subsubsection{Western blot analysis}

Sodium dodecyl sulfate-polyacrylamide gel electrophoresis (SDS-PAGE) was carried out to separate proteins with different molecular masses. Equal amount of protein $(20 \mu \mathrm{g})$ was loaded on SDS-PAGE gel and electrophoresis was conducted at $100 \mathrm{~V}$ for 30 mins followed by $120 \mathrm{~V}$ for $1 \mathrm{~h} 40 \mathrm{~min}$. The protein was transferred onto nitrocellulose (NC) membranes using a semi-dry blotting system $(25 \mathrm{~V}, 1.0 \mathrm{~A}) .10 \%$ separation gel was used for the detection of the proteins more than $30 \mathrm{kDa}$. $15 \%$ gel was used for proteins less than $30 \mathrm{kDa}$. Proteins on NC membrane were visualized by short incubation with Ponceau $\mathrm{S}$ solution. Subsequently, membrane was blocked with $5 \%(\mathrm{w} / \mathrm{v})$ milk powder in TBST buffer for $1 \mathrm{~h}$ at room temperature, followed by incubation with primary antibodies overnight at $4^{\circ} \mathrm{C}$. Secondary HRP-linked antibodies were incubated for $1 \mathrm{~h}$ at room temperature. All antibodies were diluted in $5 \%$ milk powder in TBST buffer. Protein was detected by an Intas ChemoCam Imager using ECL substrate (Bio-Rad, Hercules, USA). Antibodies used for this study are listed in Table 11. 


\subsubsection{Proteome profiler cytokine array}

Cells were seeded in a 6-well plate in $2 \mathrm{~mL}$ medium and grown to $80-90 \%$ confluency. $48 \mathrm{~h}$ after a medium change the supernatant was collected and centrifuged at $5,000 \mathrm{Xg}$ for 5 min prior to cytokine detection using Proteome Profiler Human Cytokine Array Kit (R\&D Systems, Minneapolis, USA) following the manufacturer's instructions.

\subsubsection{ELISA}

Cells were seeded in a 24-well plate in $500 \mu \mathrm{L}$ medium. $48 \mathrm{~h}$ after medium change the supernatant was collected and centrifuged at $5,000 \mathrm{Xg}$ for $5 \mathrm{~min}$ prior to detection. The levels of secreted human TNFa were measured by Quantikine ELISA kit (R\&D Systems, Minneapolis, USA). For in vivo detection, blood samples were collected and centrifuged at $4000 \mathrm{Xg}$ for $10 \mathrm{~min}$. Plasma of control and TNF $\alpha$ treated mice were measured for human and murine TNFa by Quantikine ELISA kit (R\&D Systems, Minneapolis, USA).

\subsubsection{Next generation sequencing}

\subsubsection{RNA-seq and data processing}

$5 \times 10^{5}$ CAPAN1 cells were seeded in 6-well plates. The next day, cells were treated with either TNFa (10 ng/mL; Sigma-Aldrich, St. Louis, USA) or vehicle control for $18 \mathrm{~h}$. Next, cells were harvested in TRIzol (Ambion, Kaufungen, Germany) for RNA isolation (three biological replicates). The RNA quality was checked by agarose gel electrophoresis. cDNA library was prepared using $500 \mathrm{ng}$ of total RNA and the TruSeq RNA Library Prep kit (Illumina, San Diego, USA) as per manufacturer's instructions. cDNA concentration was measured via Qubit dsDNA high sensitivity assay (Thermo Fisher Scientific, Waltham, USA). Fragment sizes were confirmed by Bioanalyzer high sensitivity DNA analysis (Agilent Technologies, Santa Clara, California, USA) prior to sequencing (single-end 50 bp) on a HiSeq2000 (Illumina, San Diego, USA). Sequencing was performed at the core facility of NGS Integrative Genomics Unit at the UMG, Göttingen.

For data analysis, the read-outs were quality-checked using FastQC v0.11.5 (https://www.bioinformatics.babraham.ac.uk/projects/fastqc/). Reads were mapped 
against reference human genome hg38 by STAR v2.5.2b (Dobin et al., 2013), Next, aligned reads were assigned to individual genes for transcript quantification by HTSeqcount version 0.10.0 (Anders et al., 2015). Differential expression analysis was performed by DESeq2 (Love et al., 2014). Genes were considered differentially expressed between treatments if they had an absolute log2-fold change of $>0.58$ and an adjusted $p$-value of $<0.05$. Principle component analysis (PCA) was determined by prcomp function in $R$ and plotted by ggplot2 in $\mathrm{R}$. pheatmap package in $\mathrm{R}$ was utilized for generating heatmaps. Gene set enrichment analysis (GSEA) was performed using $\mathrm{R}$ package clusterProfiler (Yu et al., 2012). Bioinformatic analysis was performed by X. Li (Developmental Biology, University of Göttingen, Germany).

\subsubsection{ChIP-seq library preparation and data processing}

$10-15 \times 10^{6}$ cells were cross-linked in $1 \%$ PFA (PBS-diluted) for $10 \mathrm{~min}$ at room temperature. Subsequently, cells were quenched in ice-cold glycine $(0.125 \mathrm{M})$ for $5 \mathrm{~min}$. The preparation of ChIP materials was followed by the protocol from Ford et al. (Ford et al., 2014). A Bioruptor sonicator was utilized to generate a size of 300-500 bp DNA fragments (https://ethanomics.files.wordpress.com/2012/01/chip covaris4.pdf). For the immunoprecipitation, antibodies used are listed in Table 14. Immunoprecipitated DNA was purified using the ChIP DNA Clean \& Concentrator kit (Zymo Research Europe, Freiburg, Baden-Württemberg, Germany), following the manufacture's instruction. Nextgeneration sequencing (single-end) was conducted on a HiSeq4000 platform (Illumina, San Diego, USA) to obtain at least 20 million reads per sample. Raw sequencing reads ( 100 bp) were mapped to the reference human genome (hg19) using BWA (Li and Durbin, 2010). Peak calling was performed by MACS2 (Zhang et al., 2008) to identify signal enrichment over input. Next, genes were assigned to peaks through GREAT (http://great.stanford.edu/public/html/). ChIP-seq analysis was performed by $\mathrm{T}$. Georgomanolis (Cologne Center for Genomics, University of Cologne, Germany). 


\subsubsection{Tissue microarray (TMA) staining and analysis}

Human PDAC tissue microarray (TMA) slides were obtained from the Departments of Pathology as well as General, Visceral and Pediatric Surgery of the University Medical Center Göttingen following the ethical regulations of the institute (70112108). In total, 46 matched PDAC patient samples were evaluated, with one to three cores per patient. IF staining, TMA evaluation, and data acquisition were performed as described above.

\subsubsection{Publicly available PDAC microarray data analysis}

For patient microarray expression analysis, data from Puleo et al. (Puleo et al., 2018) was downloaded from the ArrayExpress database (E-MTAB-6134). For genes associated with multiple probes, the probe with the highest average expression across all patients were used for the analysis. For gene set overrepresentation analysis in squamous and pancreatic progenitor PDAC patient tumors (Bailey et al., 2016), the differential expression was performed on the R2 platform (https://hgserver1.amc.nl/cgibin/r2/main.cgi) using the default parameters and enrichment analysis conducted using clusterProfiler as described above. Publicly available patient data analysis was performed by Lukas Klein (Department of Gastroenterology, Gl Oncology \& Endocrinology, University Medical Center Göttingen, Germany)

\subsubsection{Statistical analysis}

GraphPad Prism version 8.0.2 was used for statistical analysis (GraphPad Software, San Diego, USA). Mann-Whitney test, or unpaired Student's t-test was performed for the comparison of two groups. Log-rank test was applied for survival data. For gene expression correlation of published microarray data, the Pearson's correlation coefficient with a two-tailed $P$ value was calculated. Results were considered significant with a $P$ value below 0.05 , as indicated in the figures. 


\section{Results}

\subsection{High level of TNFa is associated with BL phenotype in PDAC}

In order to investigate the role of TNFa in PDAC progression, we first examined whether there was any correlation between TNF $\alpha$ expression and a certain tumor phenotypic identity. Therefore, we utilized a genetically engineered Kras ${ }^{G 12 D} ; p 53^{R 172 H} ; \mathrm{Pdx} C r e(\mathrm{KPC})$ mouse model, which is widely used in PDAC studies as it recapitulates human pancreatic carcinogenesis (Hingorani et al., 2005). KPC tumor sections were subjected to H\&E staining. KPC tumors were highly heterogeneous and contained both well-to-moderately differentiated (W/M) and poorly differentiated tumor contents. As shown in Figure 6A, G1 and G2 tumors presented W/M tumor features, with glandular, duct-like structures, whereas G3 and G4 tumors were lacking cellular differentiation, forming extremely poorly differentiated histological features. We then probed TNFa and cytokeratin-19 (CK19) on these W/M (G1-G2) and poorly differentiated (G3-G4) KPC tumor tissues. CK19 is a surrogate marker for tumor cells. Notably, TNFa was found to be highly expressed in poorly differentiated tumors (G3-G4) when compared with W/M (G1-G2) differentiated tumors (Figure 6B), indicating an association between TNF $\alpha$ and the poorly differentiated phenotypic state.

To further evaluate the tumor phenotype-dependent expression of TNFa, human CLA and $B L$ orthotopic mouse models were generated to investigate whether TNFa was highly expressed in $\mathrm{BL}$ tumors, which are associated with poorly differentiated tumors. To this end, immunodeficient mice were selected to allow human PDAC cells (CLA and BL) to grow inside the pancreas. The established CLA (i.e. CAPAN1 and CAPAN2) and BL (i.e. PANC1 and MiaPaCa2) cell lines were orthotopically transplanted into the pancreas of immunodeficient mice NMRI-Foxn1 ${ }^{\text {nu/nu }}$. Notably, the CLA subtype-derived tumors maintained glandular structures and formed well differentiated tumors, and the liver tissues did not form any visible metastases. In comparison, as expected, the BL tumors formed poorly differentiated tumors and liver metastases (Figure 6C). Double immunofluorescence (IF) staining of TNFa and CK19 was performed again in the CLA 
and $\mathrm{BL}$ orthotopic tumors. In line with the observation in KPC mice, TNFa was found to be highly expressed in the BL tumors compared to CLA tumors (Figure 6D).

A

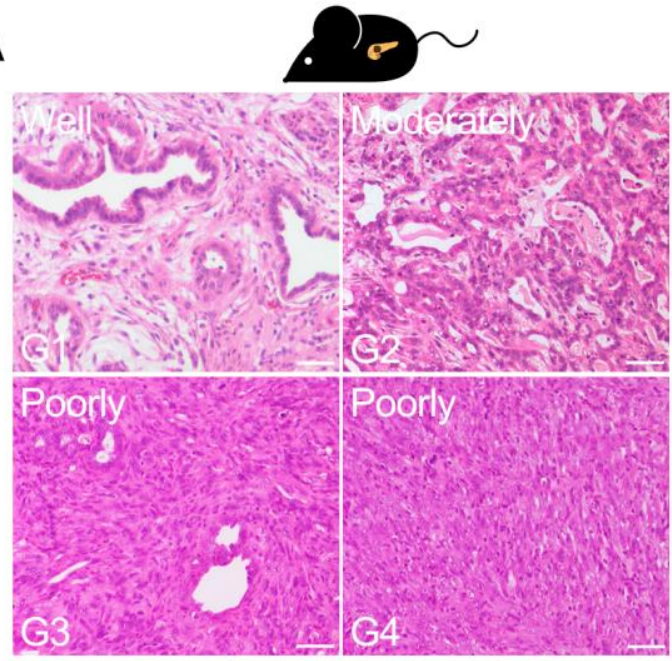

$\operatorname{Kras}^{612 D} ; p 53^{R 172 H} ; \mathrm{Cre}(\mathrm{KPC})$

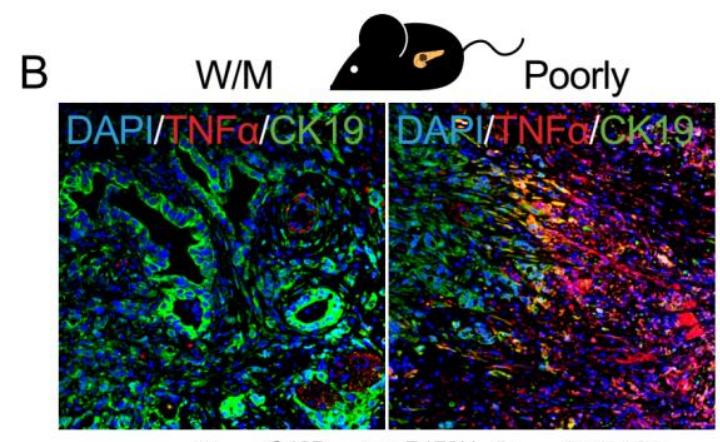

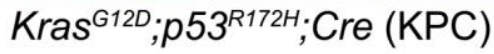

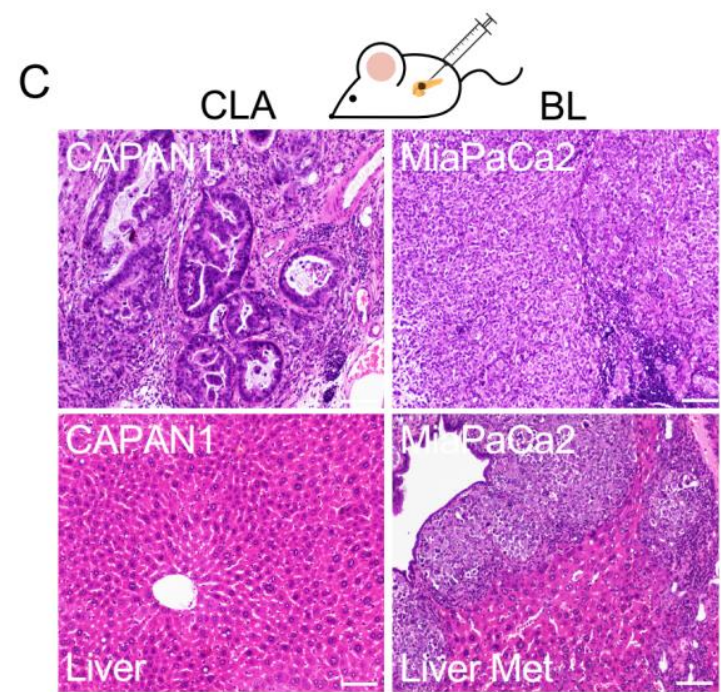

Orthotopic implantation

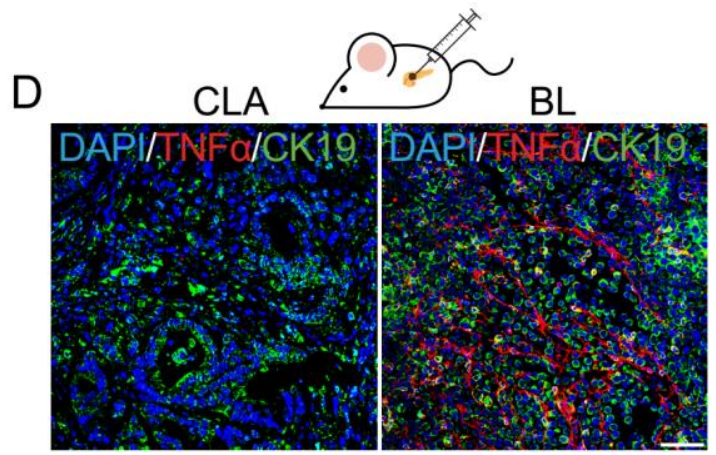

Orthotopic implantation

Figure 6. Phenotype-dependent expression of TNFa in PDAC. (A) Representative H\&E images of graded (G1 to G4) Kras ${ }^{G 12 D} ; p 53^{R 172 H} ; P d x C r e(K P C)$ tumor tissues. Scale bar: $20 \mu \mathrm{m}$. (B) Immunofluorescence (IF) staining of TNFa (red) and cytokeratin 19 (CK19) in well-tomoderately differentiated (W/M) as well as poorly differentiated tumors of KPC mice. Scale bar: $50 \mu \mathrm{m} . \mathrm{n}=5$ in W/M; $\mathrm{n}=5$ in poorly. (C) Representative images of H\&E staining in pancreatic tumors and liver tissues derived from orthotopically implanted classical (CLA) CAPAN1 as well as basallike (BL) MiaPaCa2 mouse models. Scale bar: $20 \mu \mathrm{m}$. (D) IF staining of TNFa (red) and CK19 (green) in the tumor tissues as indicated in (C). Scale bar: $50 \mu \mathrm{m} . n=9$ in CLA; $n=10$ in BL. 
To further validate whether high levels of TNFa determines subtype-specificity, we used histologically characterized W/M and poorly differentiated resected PDAC patient tumors. Notably, we observed high TNFa levels particularly in the poorly differentiated tumors compared to W/M PDAC (Figure 7A and 7B). Collectively, these data indicate that high levels of TNFa are associated with highly aggressive BL phenotypic state.

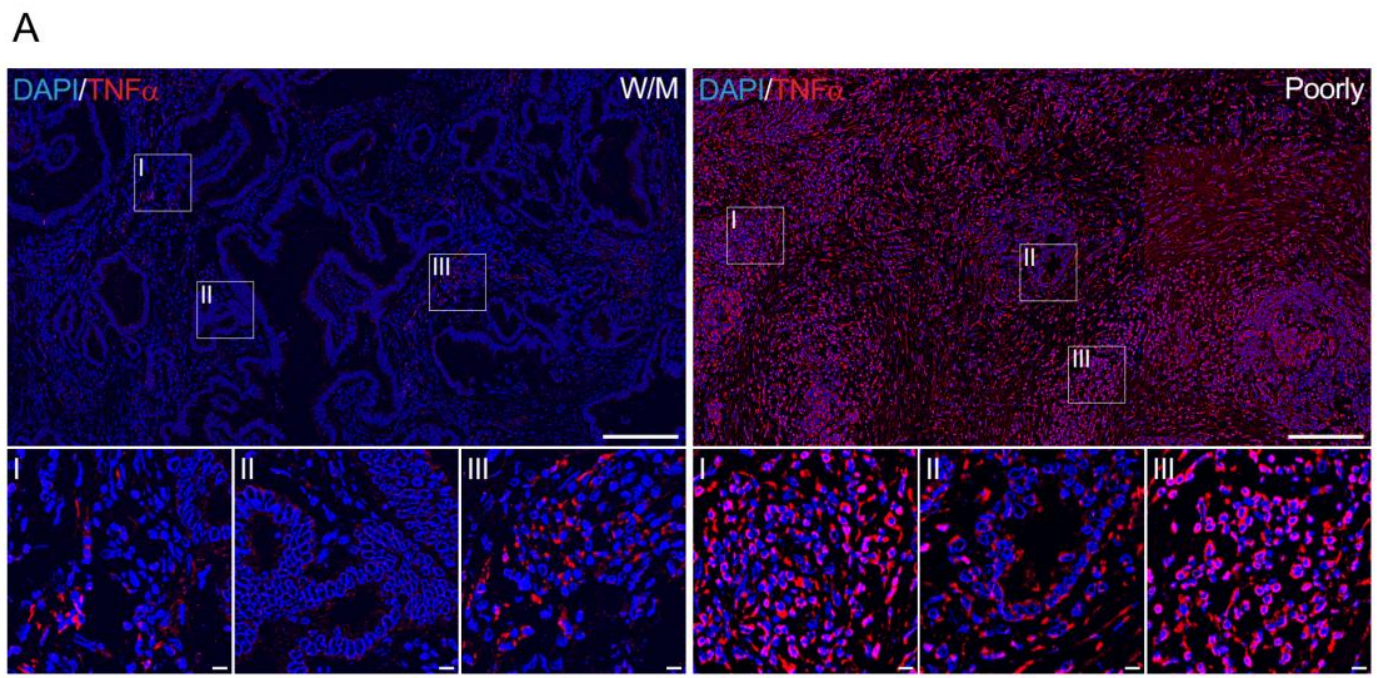

B

Human PDAC tumors

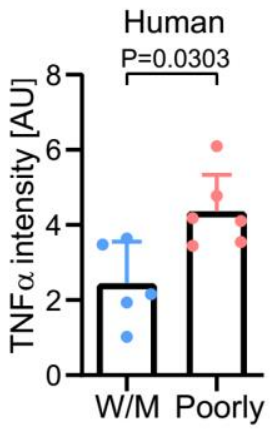

Figure 7. TNFa is highly expressed in poorly differentiated PDAC patient tumors. (A) representative tile scan images of IF staining of TNFa in W/M and poorly differentiated PDAC patient tumors. I-III show higher magnification images. Scale bar: whole section (top): $2 \mathrm{~mm}$; higher magnification images (bottom): $10 \mu \mathrm{m}$. (B) Quantification of TNFa intensity in (A). Scatter plots show values in arbitrary units (AU) of each patient, as well as means \pm s.d. as bar graphs. Mann-Whitney test was used for statistical analysis. $n=5$ in $W / M ; n=6$ in poorly.

\subsection{TNFa promotes CLA to BL phenotypic switch in PDAC}

\subsubsection{TNFa promotes poorly differentiated phenotypic state in vivo}

Next, we sought to investigate whether TNFa promotes tumor aggressiveness in PDAC. Therefore, CLA (i.e. CAPAN1) subtype cells were orthotopically implanted in mice and treated with exogenous TNFa. As detailed in figure 8A, immunodeficient NMRI Foxn1 ${ }^{\text {nu/nu }}$ 
mice were implanted with CAPAN1 cells into the pancreas. Three weeks after transplantation, as it approached the time that tumor started to form, 3D ultrasound was used weekly to evaluate the tumor growth until mice developed a decently sized tumor (approx. $6 \mathrm{~mm}$ in diameter) (Figure 8B). Mice were administrated TNFa or vehicle control intraperitoneally (i.p.) three times per week. At the endpoint (three weeks of TNFa treatment), mice were sacrificed for tissue isolation.

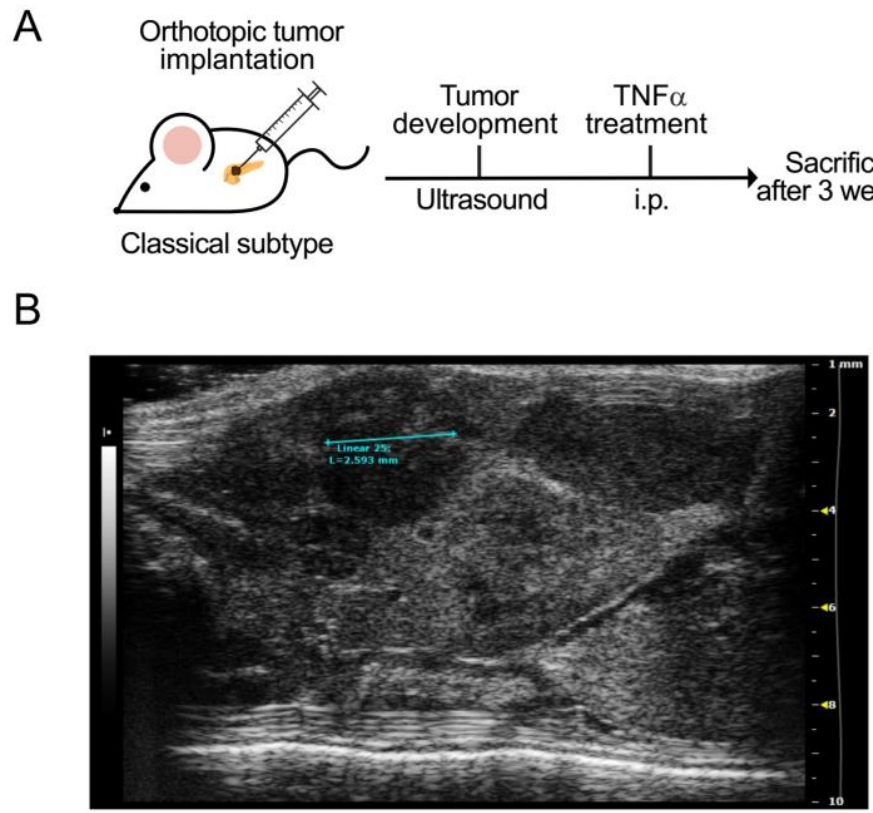

Figure 8. Schematic of orthotopically implanted PDAC cells into the pancreas. (A) An illustration of the experimental design. 10-weeks old male immunodeficient NMRI-Foxn $1^{\text {nu/nu }}$ mice were orthotopically implanted with $1 \times 10^{6}$ CLA cell line CAPAN1 into the pancreas. Three weeks after transplantation, (B) ultrasound was performed weekly to evaluate the tumor growth. Treatment began when the tumor diameter reaches approx. $6 \mathrm{~mm}$. TNF $\alpha(0.4 \mathrm{mg} / \mathrm{kg}$ body weight) was injected intraperitoneally (i.p.) three times a week, using aqua dest. as control. Mice were sacrificed after three weeks of treatment. Tissues were collected including entire pancreatic tumor, spleen, liver, lung, and blood samples.

Next, tumor tissues harvested from vehicle control (VC) and TNFa-treated mice were subjected to H\&E staining. In order to have an overview of the tumor histology, whole slide scans of the H\&E sections were performed. Representative tile images as well as 
selected tumor areas at a higher magnification are shown in Figure 9A. In general, control tumors exhibited a W/M differentiated phenotype that fully recapitulates the CLA PDAC subtype identity. However, the proportion of poorly differentiated tumor area significantly increased in TNFa-treated mice, which is a sign for the BL subtype, as quantified in Figure 9B. Duct-like structures were prominent in the VC tumors compared to more separated and irregular structures in the TNFa-treated tumors, suggesting that TNFa switched CLA to the highly aggressive BL phenotypic state.

A

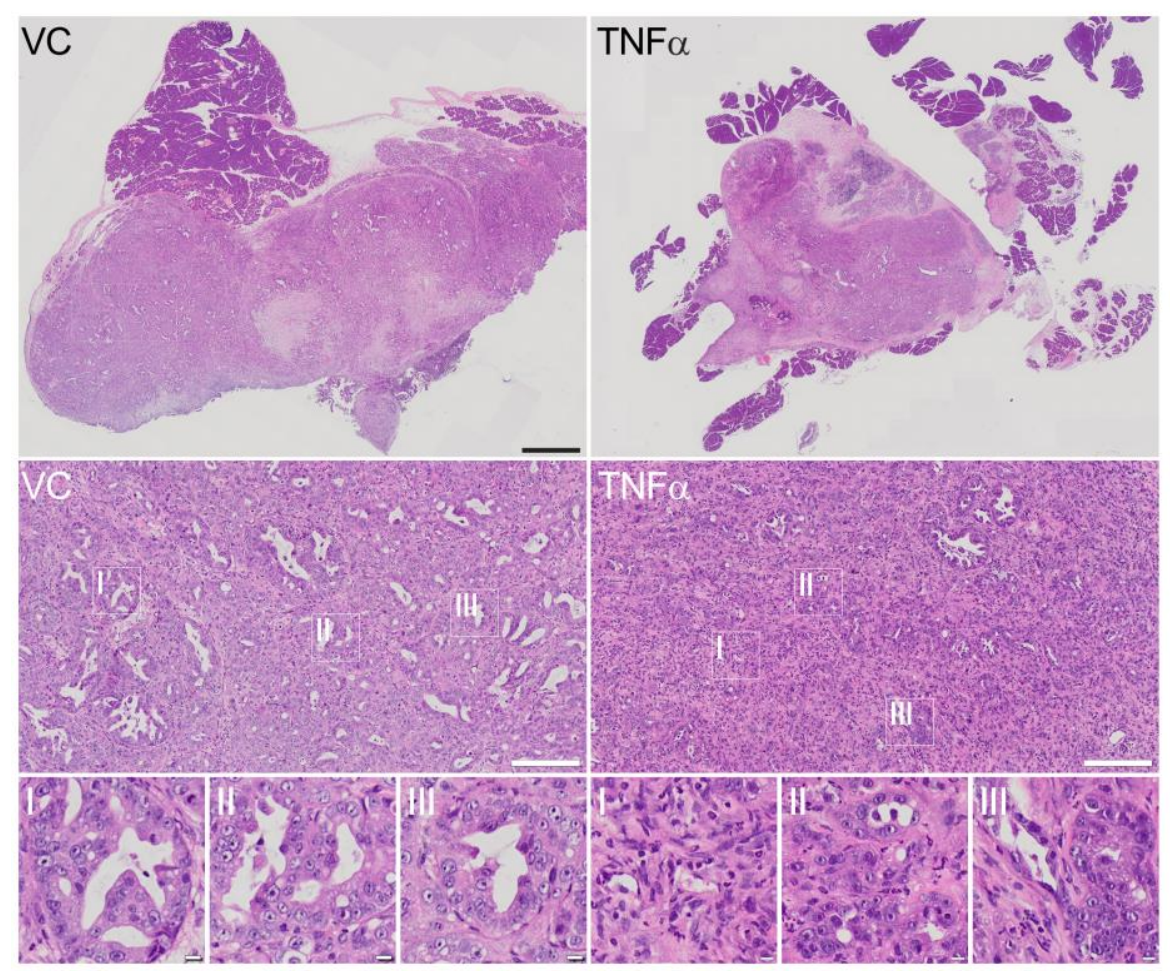

B

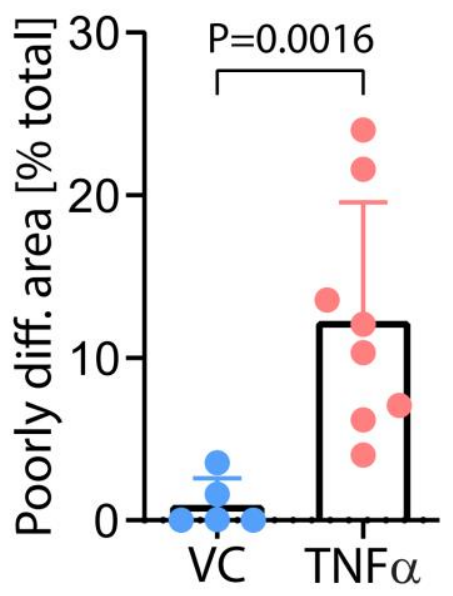

Figure 9. TNFa promotes W/M to poorly differentiated phenotype. (A) Representative tile scan images of H\&E staining in pancreatic tumor tissues from vehicle control (VC) and TNFatreated orthotopically implanted mice (CAPAN1). I-III show higher magnification images. Scale bar: whole section (top): $2 \mathrm{~mm}$; representative images (middle): $200 \mu \mathrm{m}$; higher magnification images (bottom): $10 \mu \mathrm{m}$. (B) Scatter plots show quantification of poorly differentiated tumor area as a percentage of total tumor area in VC and TNFa-treated mice, as well as means \pm s.d. as bar graphs. $n=5$ in VC; $n=8$ in TNFa. Mann-Whitney test was used for statistical analysis. 
To further validate our observations, IF staining was performed for Keratin-81 (KRT81) and amylase in the tumors developed from VC and TNFa-treated mice. Of note, KRT81 is an established clinical marker that is specifically expressed in the BL tumors (Noll et al., 2016). Additionally, PDAC patients showing high levels of KRT81 have a lower survival rate (Noll et al., 2016). Amylase was used to stain for normal pancreatic acinar cells, which allowed us to distinguish tumor cells from normal tissue. We observed a significant induction of KRT81 expression in the tumor core as well as peritumoral regions (amylase ${ }^{+}$) following TNFa treatment (Figure 10), consistent with the tumor histology.

Interestingly, except for the induced BL invasive phenotype, abundant infiltration of CD45+ immune cells were found in the TNFa-treated tumors. In contrast, VC tumors barely have $\mathrm{CD}_{45}{ }^{+}$cells present (Figure 11). Collectively, these data suggest that TNFa promotes a poorly differentiated/BL phenotype and immune cell infiltration in PDAC.

A

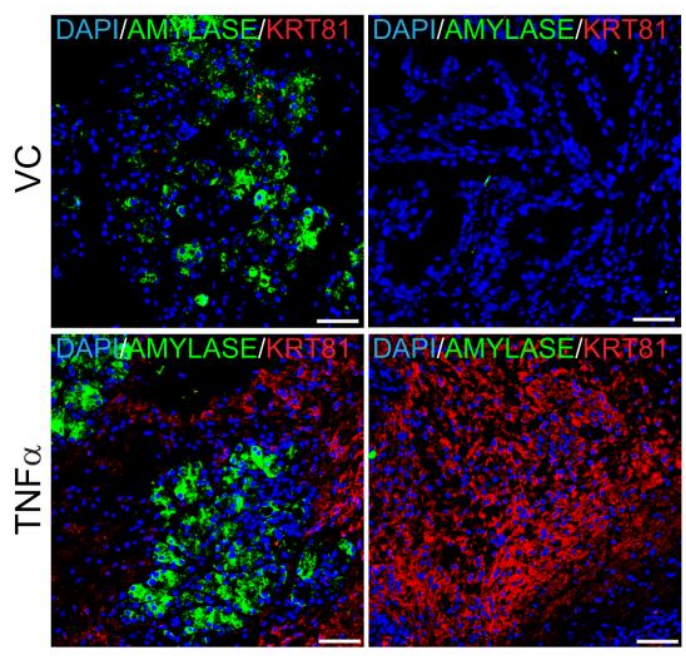

B

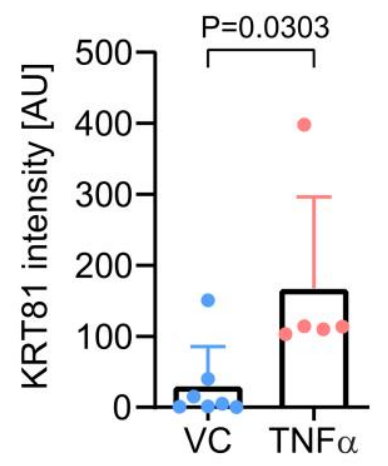

Figure 10. TNFa treatment leads to high level of of KRT81 in vivo. (A) Representative IF images of KRT81 (red) and amylase (green) in orthotopic CAPAN1 VC and TNFa treated pancreatic tumor tissues. scale bar: $50 \mu \mathrm{m}$. (B) Quantification of KRT81 in (A). Scatter plots show values in arbitrary units (AU) as an average per animal, as well as means \pm s.d. as bar graphs. 10 images were evaluated per animal. Mann-Whitney test was used for statistical analysis. $n=7$ in VC; $n=5$ in TNFa. 
A

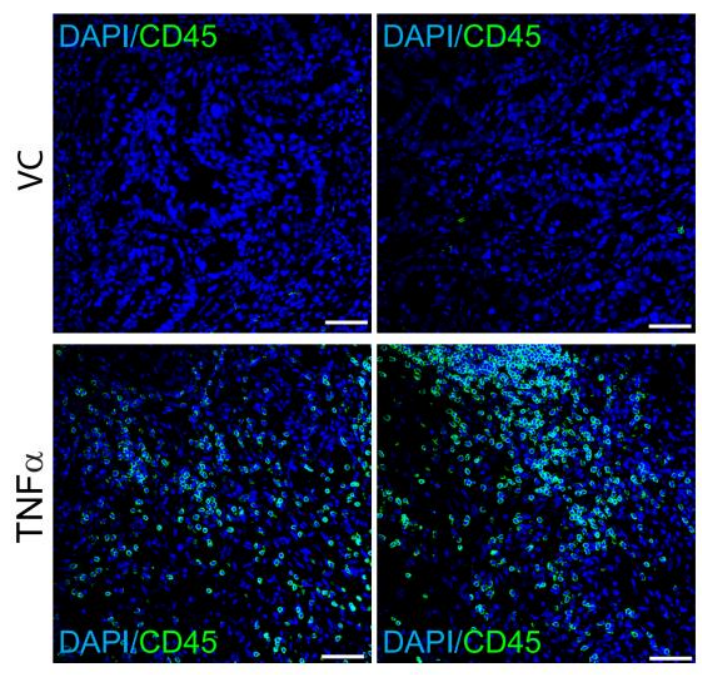

B

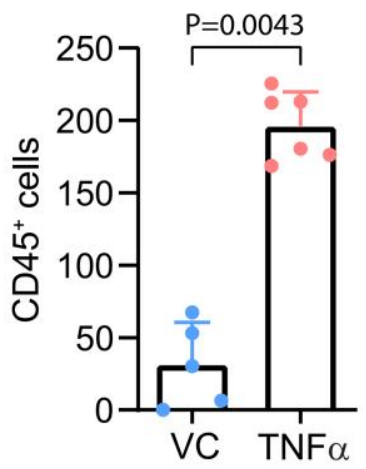

Figure 11. TNFa treatment induces $\mathrm{CD}^{4} 5^{+}$immune cell infiltration. (A) Representative IF images of CD45 (green) in orthotopic CAPAN1 VC and TNFa treated tumor tissues. Scale bar: $50 \mu \mathrm{m}$. (B) Quantification of CD45 cells in (A). Scatter plots show average values per animal of counts per field of view as well as means \pm s.d. as bar graphs. 10 images per animal were evaluated. Mann-Whitney test was used for statistical analysis. $n=5$ in VC; $n=6$ in TNFa.

\subsubsection{TNFa promotes invasive behavior of CLA PDAC cells}

To understand the mechanism underlying the observed phenotypic switch in vivo, we performed a 3D-transwell invasion assay in CLA cells following TNFa treatment. The number of invading cells was significantly induced in presence of TNFa in both CLA cell lines, i.e. CAPAN1 and CAPAN2 (Figure 12A and 12B). Additionally, the increased invasive capacity was associated with an induction of EMT marker ZEB1, concomitant with a reduction of differentiation marker KLF5. The most prominent effect was seen after $48 \mathrm{~h}$ of the TNFa treatment (Figure 12C). 
A

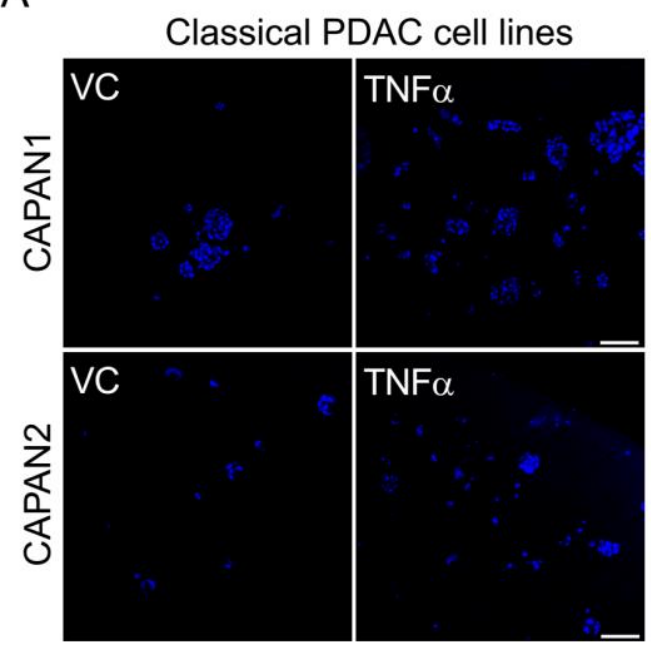

B

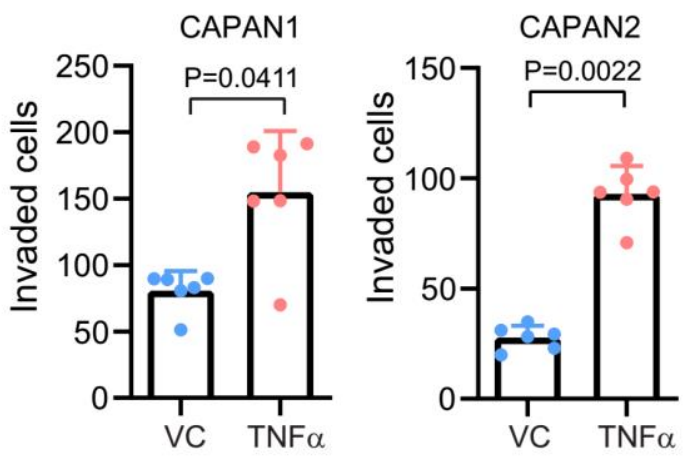

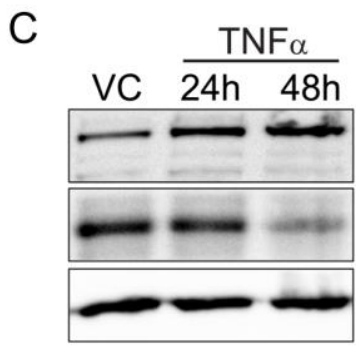

CAPAN1

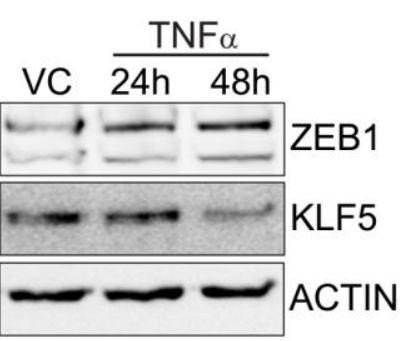

CAPAN2

Figure 12. TNFa increases the invasive capacity of CLA cells. (A) 3D-transwell invasion assay of CLA cell lines (CAPAN1 and CAPAN2) treated with TNFa $(10 \mathrm{ng} / \mathrm{mL})$, or aqua dest. as vehicle control (VC). Representative images of DAPI-stained invaded CAPAN1 and CAPAN2 cells. Scale bar: $50 \mu \mathrm{m}$. (B) Quantification of invaded cells as shown in (A). Scatter plots show average values per technical replicate of counts per F.o.V. as well as means \pm s.d. as bar graphs. Mann-Whitney test was used to determine the statistical significance. $n=3$. (C) Western blot analysis of ZEB1, KLF5 upon TNFa or VC treatment for $24 \mathrm{~h}$ and $48 \mathrm{~h}$ in CLA cells CAPAN1 and CAPAN2. Actin was used as a loading control. Representative of three independent experiments.

\subsubsection{TNFa induces CLA to BL subtype switch by transcriptional reprogramming of lineage-specific genes}

To gain further insight into the molecular mechanisms underlying the TNFa-mediated subtype switch, we performed RNA sequencing using CLA CAPAN1 cells treated with 
TNFa or vehicle control. Gene set enrichment analysis (GSEA) revealed a significant enrichment of EMT and inflammatory response hallmarks in TNFa-treated samples (Figure 13A), consistent with our previous observations of TNFa-treated orthotopic tumors as well as in vitro experiments (Figure 9, Figure 11, and Figure 12). Additionally, gene sets linked to the invasive PDAC subtype such as 'KRAS signaling down' and 'Myogenesis' (Miyabayashi et al., 2020) were also found to be enriched in the TNFatreated samples (Figure 13B), whereas gene sets involved in 'Fatty acid metabolism', 'Adipogenesis', and 'Myc targets V1' were enriched in control samples (Figure 13B). To determine whether TNFa-altered gene signatures associated with a particular PDAC subtype, we utilized the publicly available CLA-related gene sets, as previously reported (Maurer et al., 2019). We found that TNFa treatment led to a reduction in the expression of most CLA-specific genes (Figure 13C). The genes that were upregulated coinciding with a known $\mathrm{BL}$ signature, as well as downregulated genes coinciding with CLA were shown in volcano plot (Figure 13D). Taken together, these data suggest that TNFa promotes a BL phenotype in PDAC cells. 
A

Hallmark epithelialmesenchymal transition
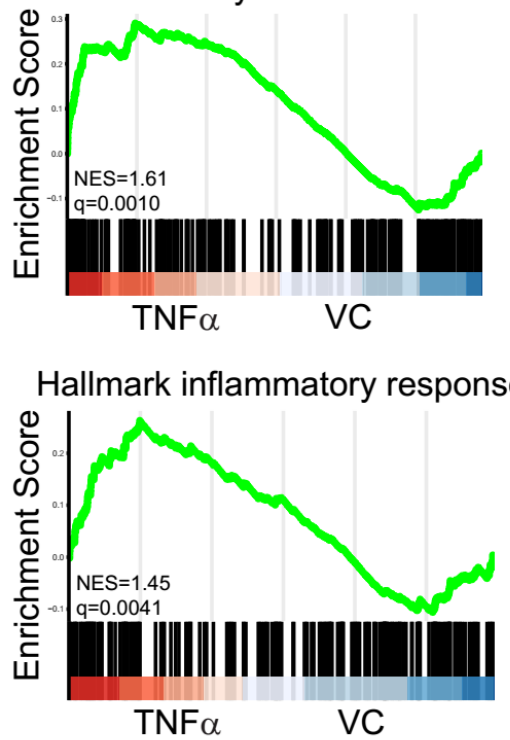

C

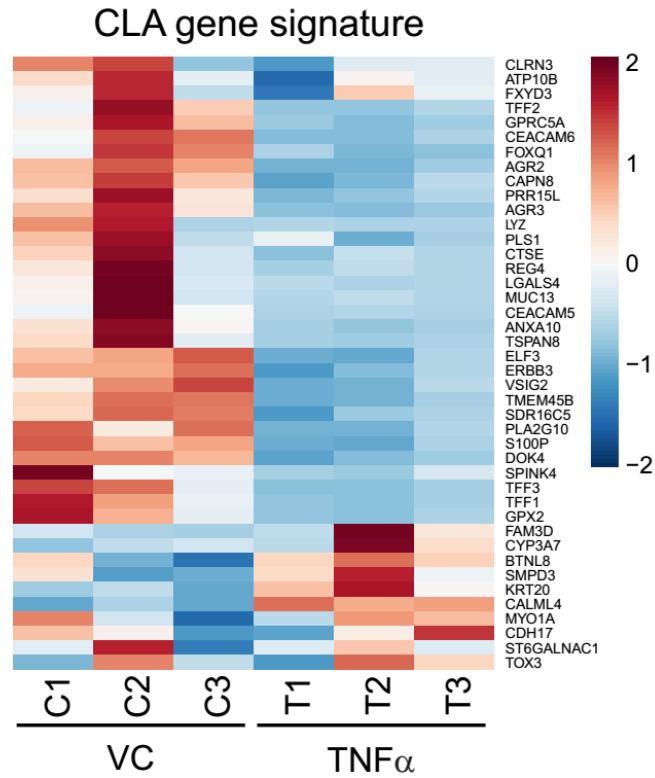

B

TNF $\alpha$ vs. VC

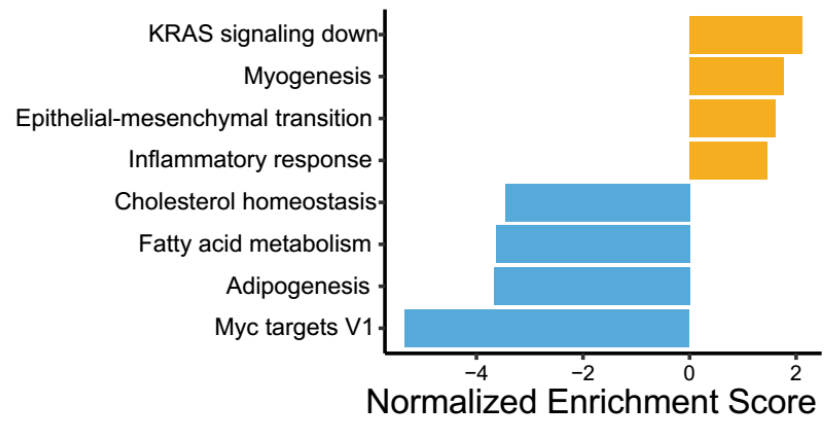

$\mathrm{D}$

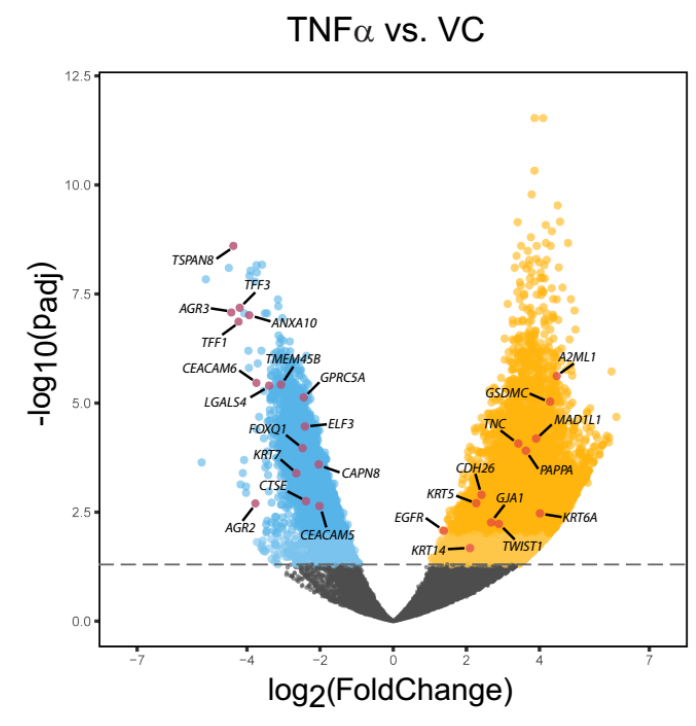

Figure 13. TNFa induced PDAC subtype-specific gene regulatory network. (A) Enrichment plots from gene set enrichment analysis (GSEA) in 18h TNFa-treated (10 ng/mL) versus vehicle control (VC) samples in CLA CAPAN1 cells. Normalized enrichment scores (NES) and q-values are indicated. (B) Normalized enrichment scores of selected Hallmark gene sets from the Molecular Signature Database in CAPAN1 cells with TNFa versus VC treatment. (C) Heatmap displays expression of CLA-associated genes as defined by Maurer et al. (Maurer et al., 2019) in CAPAN1 cells in the presence or absence of TNFa. (D) Volcano plot of differential expression 
analysis. Upregulated genes coinciding with known BL markers and downregulated genes coinciding with CLA are plotted in red.

\subsection{Molecular subtypes of PDAC determine distinct tumor microenvironment}

\subsubsection{BL-specific immune cells are source of TNFa in PDAC TME}

Based on the earlier observation that TNFa was preferably expressed in the poorly differentiated tumors (chapter 3.1), we initially hypothesized that the BL PDAC cells might be the source of TNFa. Thus, TNFa expression was examined in CLA and BL cells by flow cytometry. Despite the pivotal role of TNFa in vivo, we did not find significant levels of TNFa in either CLA (i.e. CAPAN1 and CAPAN2) or BL cells (i.e. PANC1 and MiaPaCa2) (Figure 14A). For further validation, human-specific TNFa ELISA was performed in the supernatant from these cells. Consistently, TNFa expression was not detected in all the cell lines (Figure 14B).

A

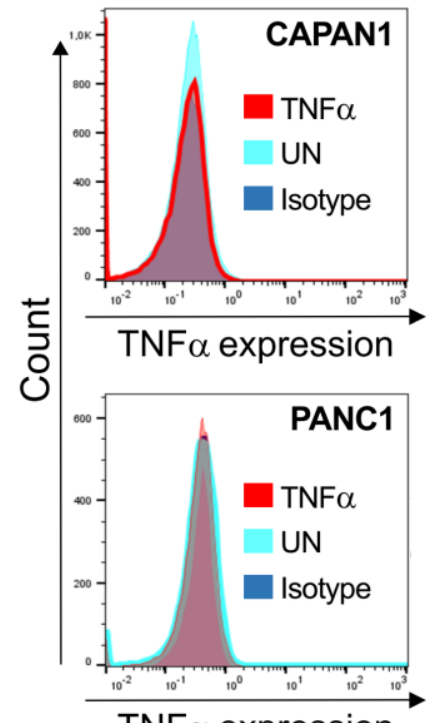

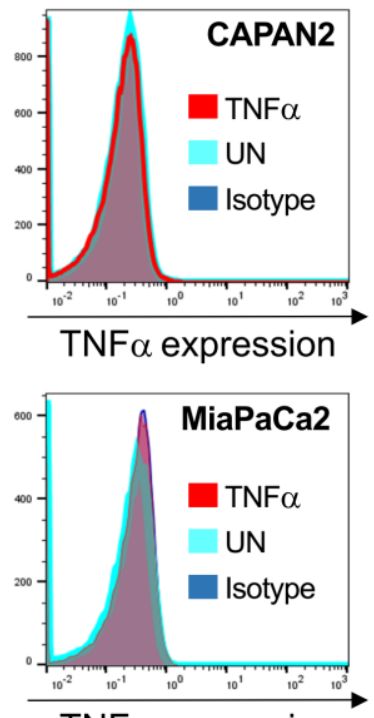

TNF $\alpha$ expression

B

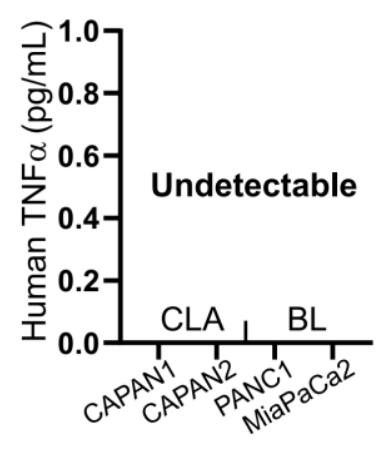

Figure 14. TNFa is not produced by PDAC cell lines. (A) Flow cytometry analysis was performed for TNFa in CLA cells CAPAN1 and CAPAN2, as well as BL cells PANC1 and 
MiaPaCa2, using anti-TNFa antibody, without any staining (UN), and isotype control (IgG1א). Counts of gated cells are shown against fluorescence intensity, which is linked to TNFa expression. $n=3$. (B) Concentrations of TNFa in cultured CLA CAPAN1 and CAPAN2 as well as BL PANC1 and MiaPaCa2 cells as measured by human-specific ELISA. $n=2$.

Given that none of the PDAC cell lines produced TNFa, we considered that inflammatory TME might be the source of TNFa production in PDAC tumors. Thus, human- and murinespecific ELISA in was carried out in the plasma-derived from the above-mentioned CLA and BL orthotopically transplanted mice (Figure 6). Notably, human TNFa secretion was not detected in either CLA or BL plasma samples (Figure 15A). Strikingly, murine TNFa was detected only in BL plasma (Figure 15B). Additionally, TNFa expression was measured in the previously mentioned TNFa-treated CLA mouse model (Figure 8). Human TNFa was able to be detected in the TNFa-treated mice as expected (Figure 15C), since human recombinant TNFa was administrated in these mice. Besides, we noted that murine TNFa could be detected as well in the TNFa treated mice (Figure 15D).

Together, human TNFa was not detectable in the PDAC cell lines as well as in the plasma from CLA and BL tumor bearing mice. However, murine TNFa was detectable in the BL and induced the TNFa-treated CLA plasma samples, suggesting that TNFa originated from the host inflammatory TME.

A

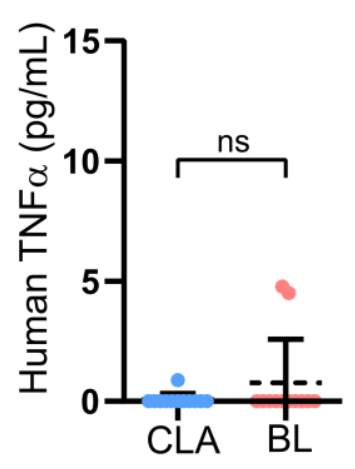

B

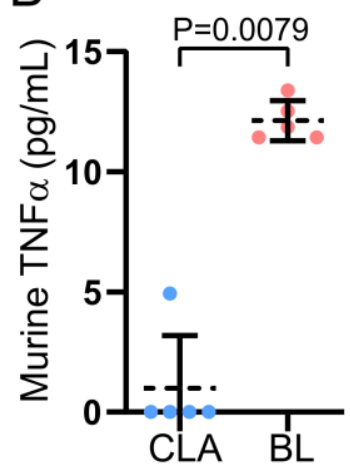

C

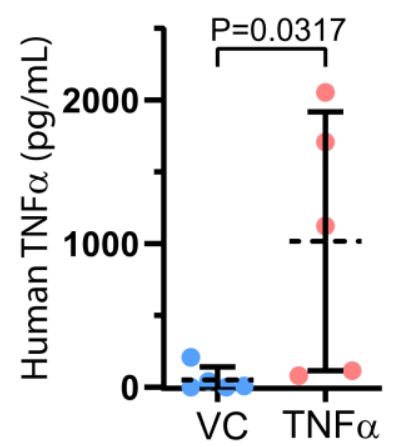

$\mathrm{D}$

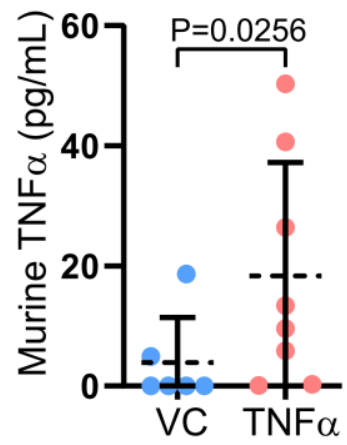

Figure 15. Host immune inflammatory tumor microenvironment produces TNFa in the $B$ PDAC subtype. (A and B) Human-specific (A) and murine-specific (B) TNFa ELISA in plasma samples isolated from mice orthotopically implanted with CLA and BL cells. Scatter plots show 
values of each animal as well as means \pm s.d. as bar graphs. Mann-Whitney test was used to determine the statistical significance. (A) $n=12$. (B) $n=5$. (C and D) Human-specific (C) and murine-specific (D) TNFa ELISA in plasma samples isolated from VC and TNFa-treated CLA mice. Scatter plots show values of each animal as well as means \pm s.d. as bar graphs. Mann-Whitney test was used to determine the statistical significance. (C) VC, $n=5$; TNFa, $n=5$. (D) $V C, n=6$, TNFa, $n=8$.

\subsubsection{Subtype-dependent immune cell infiltration}

To find out whether immune cells in the inflammatory TME were the source of TNFa, we performed IF staining of CD45 in the CLA and BL orthotopic tumors. Notably, CD45 ${ }^{+}$cells were highly present in the $B L$ orthotopic tumors compared to the CLA ones (Figure 16A). Since macrophages are known to be abundant in the PDAC TME and associated with poor survival (Bailey et al., 2016; Hessmann et al., 2020; Puleo et al., 2018), we sought to further examine the expression of the macrophage marker CD68 in these tumor tissues as well as the TNFa-treated tumors where an induction of $\mathrm{CD}_{4} 5^{+}$cells was seen (Figure 11). Notably, $B L$ orthotopic tumors showed higher recruitment of $C D 68^{+}$cells compared to CLA orthotopic tumors (Figure 16B). Similarly, $\mathrm{CD} 68^{+}$cells were found to be strongly induced upon TNFa treatment compared to VC (Figure 16C). Since these CD45 $/{ }^{+} \mathrm{CD}^{+} 8^{+}$ cells and TNFa are both more prominent in the BL tumors, indicating that TNFa is most likely produced by the immune cells, especially $\mathrm{CD} 68^{+}$macrophages. 
A

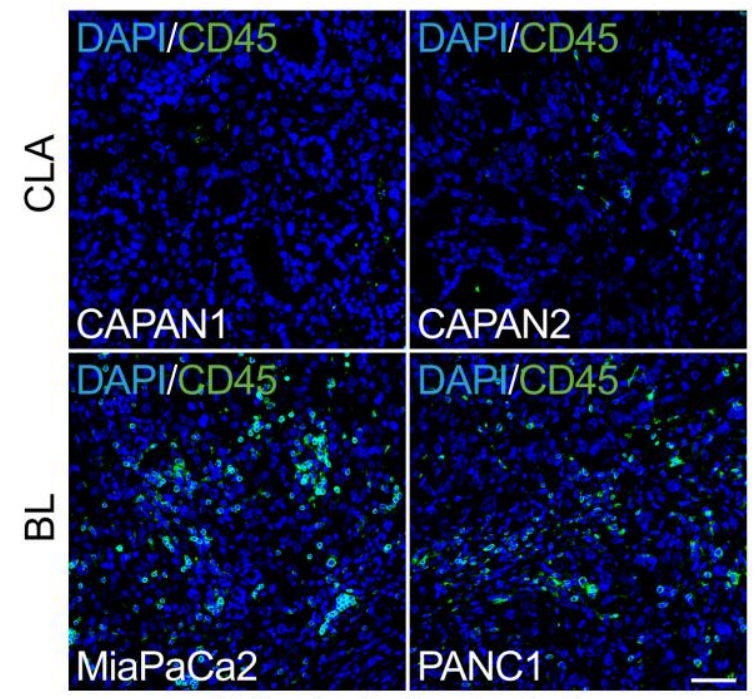

Orthotopic implantation
B
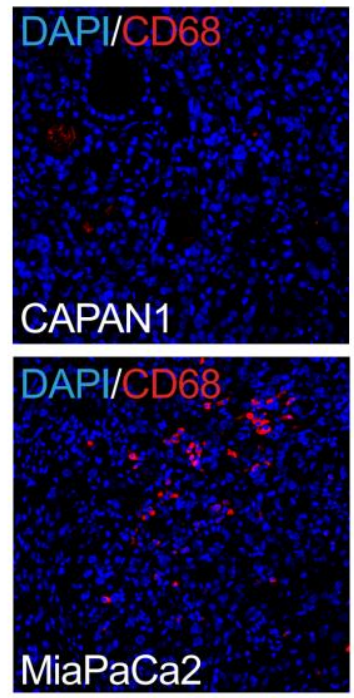

Orthotopic implantation
C
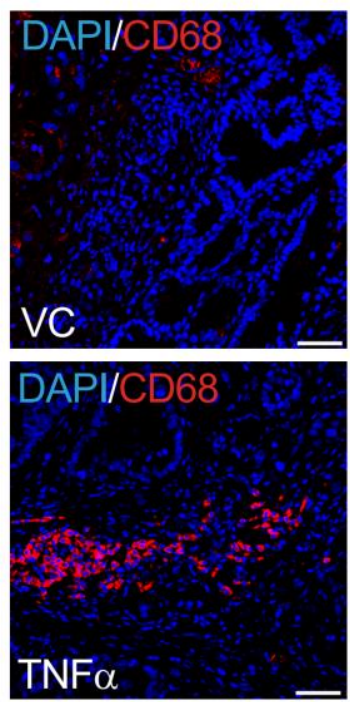

Orthotopic implantation

Figure 16. $\mathrm{CD}^{+} 5^{+}$and $\mathrm{CD} 68^{+}$cells are highly infiltrated in the $\mathrm{BL}$ orthotopic tumors. (A) Representative IF staining of CD45 (green) in CLA (CAPAN1 and CAPAN2) and BL (PANC1 and MiaPaCa2) orthotopic tumors. Scale bar: $50 \mu \mathrm{m} . n=5$ in $C L A ; n=5$ in BL. (B) IF analysis of CD68 (red) in CLA (CAPAN1) and BL (MiaPaCa2) orthotopic tumors. $n=5$ in CLA; $n=5$ in BL. (C) representative IF images of CD68 (red) in VC and TNFa-treated CLA CAPAN1 orthotopic tumors. Scale bar: $50 \mu \mathrm{m} . \mathrm{n}=6$ in VC; $\mathrm{n}=6$ in TNFa-treated.

\subsubsection{Subtype-specific intrinsic factors determine immune cell recruitment}

To understand the mechanism of how immune cells were recruited into the BL TME and its potential mediators, we first compared the cytokine expression profile between CLA and BL cell lines by utilizing publicly available RNA-seq data (GSE64560). Several cytokines were found to be upregulated in the BL cells, including CCL2, which is known to be associated with macrophages recruitment (Nagarsheth et al., 2017) (Figure 17A). Next, we used an unbiased approach via the Proteome Profiler Human Cytokine Array Kit to detect cytokine production in human CLA and BL PDAC cell lines. Consistently, CCL2 was observed to be highly upregulated in BL PANC1 and PT45P1 cell lines compared to CLA CAPAN1 and CAPAN2 cells (Figure 17B). 
As CCL2 expression correlated with the BL subtype in PDAC cell lines, we next sought to elucidate whether there was a correlation between CCL2 and subtype identity in PDAC patient tumors. Hence, we analyzed CCL2 status in the Bailey-defined pancreatic progenitor (or CLA) and squamous (or BL) subtypes (Bailey et al., 2016). In line with the association of CCL2 to the BL PDAC cells, CCL2 was found to be highly enriched in the squamous (i.e. BL) subtype compared to pancreatic progenitor (i.e. CLA) tumors (Figure 17C). Next, the association of CCL2 and $C D 68^{+}$macrophages was verified in a publicly available microarray dataset of PDAC patient tumors (Puleo et al., 2018). We found that CCL2 correlated positively with CD68 (Figure 17D). Taken together, these results indicate that high expression of $\mathrm{CCL} 2$ correlates with $\mathrm{CD} 68^{+}$macrophage infiltration in $\mathrm{BL}$ tumors.

A

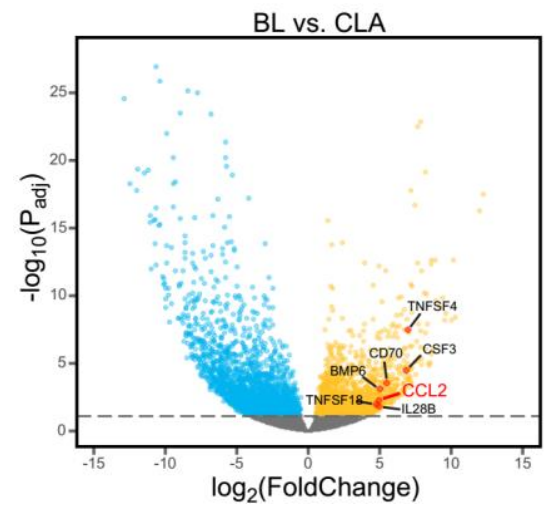

B

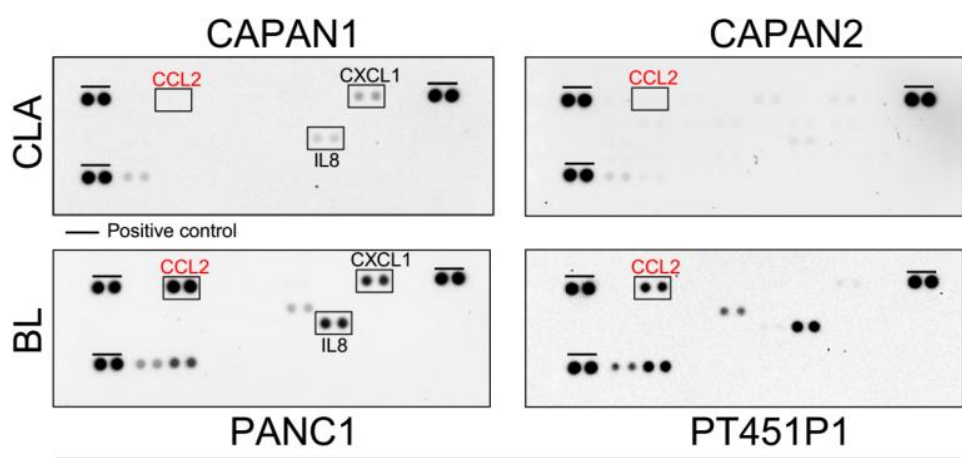

Human PDAC cell lines

D

C
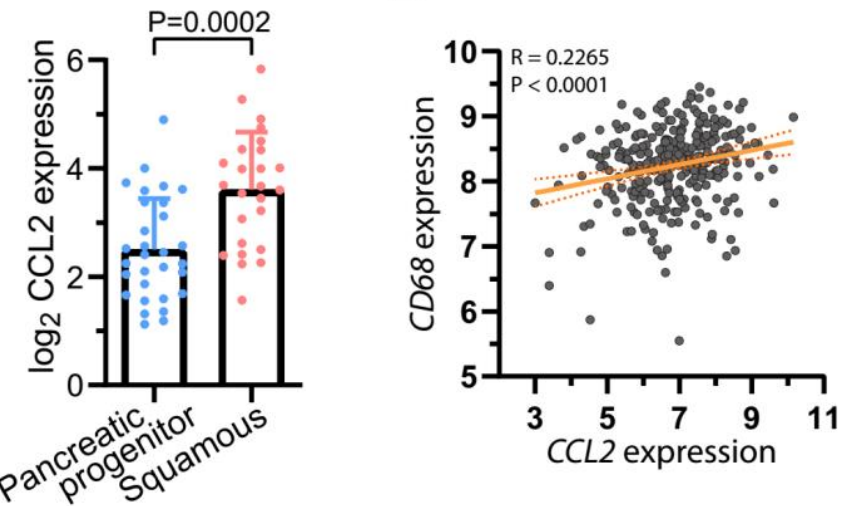

Figure 17. CCL2 is linked to BL PDAC subtype and associates with $\mathrm{CD} 68^{+}$macrophages. (A) Volcano plot of differential gene expression between CLA (CAPAN1 and CAPAN2) and BL 
(PANC1 and MiaPaCa2) PDAC cell lines. Publicly available RNA sequencing data (GSE64560; Diaferia et al., 2016) was reanalyzed by F. Wegwitz (Department of Gynecology and Obstetrics, UMG). Cytokines significantly upregulated in BL cells compared to CLA cells are highlighted. (B) Expression of cytokines detected by Proteome Profiler Human Cytokine Array Kit. CLA CAPAN1 and CAPAN2 as well as BL cells PANC1 and PT45P1. Unlabeled black spots with lines indicate for positive control. (C) Expression of CCL2 in well-defined pancreatic progenitor $(n=30)$ and squamous ( $n=25$ ) patients (Bailey et al., 2016). Data was retrieved by L. Klein (Department of Gastroenterology, GI Oncology \& Endocrinology, UMG) using R2, an online platform for genomic dataset analysis (https://hgserver1.amc.nl/cgi-bin/r2/main.cgi). Mann-Whitney test was used to determine the statistical significance. (D) Correlation analysis of CCL2 and CD68 in 309 PDAC patient tumors (Puleo et al., 2018). Data was obtained from the ArrayExpress database (E-MTAB6134). RMA normalized probe intensity were plotted. A linear regression with $95 \% \mathrm{Cl}$ is shown in orange.

\subsection{Distinct AP1 transcription factors determine PDAC subtype identity}

\subsubsection{Mutually exclusive expression of JUNB/AP1 and cJUN/AP1 in PDAC}

To elucidate the mechanism of the observed cytokine upregulation, we analyzed publicly available patient microarray data (Puleo et al., 2018), and found a significant enrichment of the TNFa signaling pathway and inflammatory AP1 signaling exclusively in BL patients (Figure 18A). Therefore, we speculated that there might be a relevance of the AP1 pathway to the observed inflammatory $\mathrm{BL}$ phenotype. Hence, we examined the expression of JUN/AP-1 family transcription factors (TFs) in CLA and BL cell lines. Strikingly, JUNB was found to be highly enriched in CLA cell lines, whereas cJUN was restricted into $\mathrm{BL}$ cells (Figure 18B). This data was further confirmed by IF staining of JUNB and CJUN in CLA and BL orthotopic tumors. Figure 18C shows high level of JUNB in CLA tumors, while cJUN was enriched in BL tumors. Next, we sought to examine whether the exclusivity of JUNB and cJUN was also reflected in patient tumors. Thus, we performed double IF staining of cJUN or JUNB together with E cadherin (ECAD) in tissue microarrays (TMA) of 46 PDAC patients. ECAD is an important molecule for cell adhesion 
and highly expressed in the CLA subtype. Of note, cJUN was abundant in the area where ECAD was negative. Conversely, JUNB was highly co-localized with ECAD+ cells in the differentiated areas, whereas cJUN was absent (Figure 18D, 18E, and 18F). Collectively, our results show that JUNB is highly expressed in the CLA well-differentiated compartment, while cJUN is enriched in BL tumors.

A

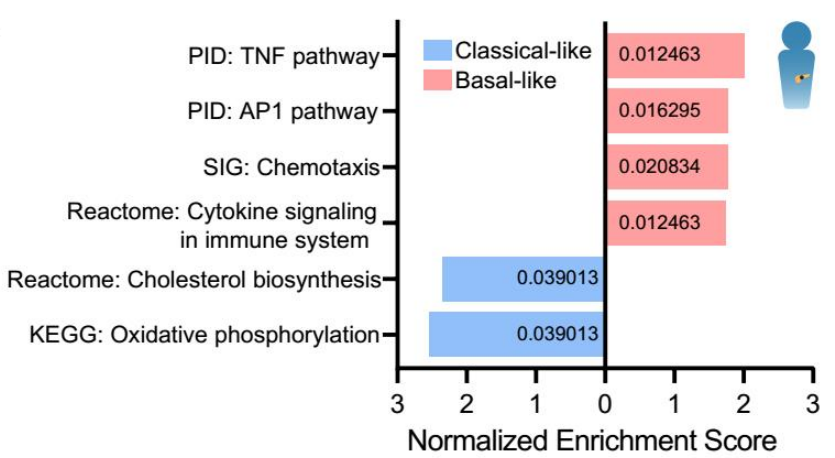

C

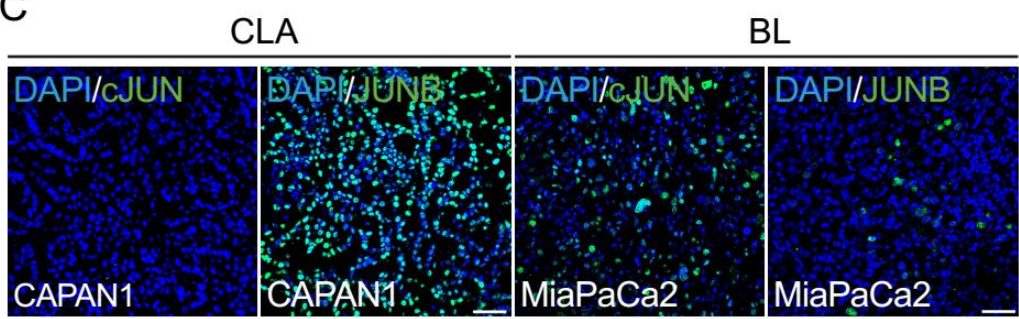

Orthotopic implantation

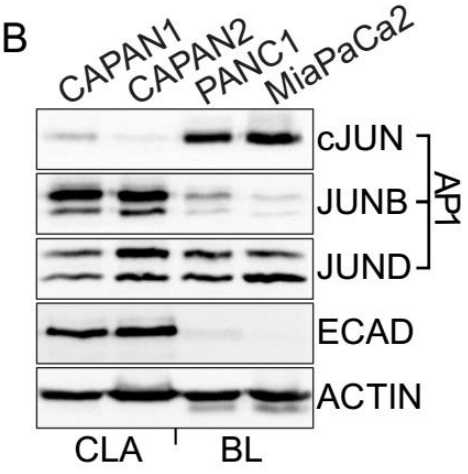

D
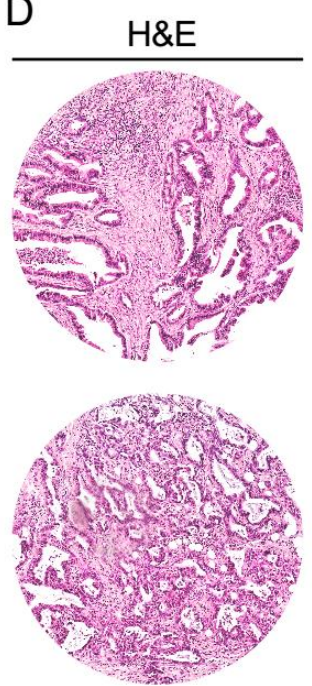

TMA ( $\mathrm{n}=46$ patients $)$
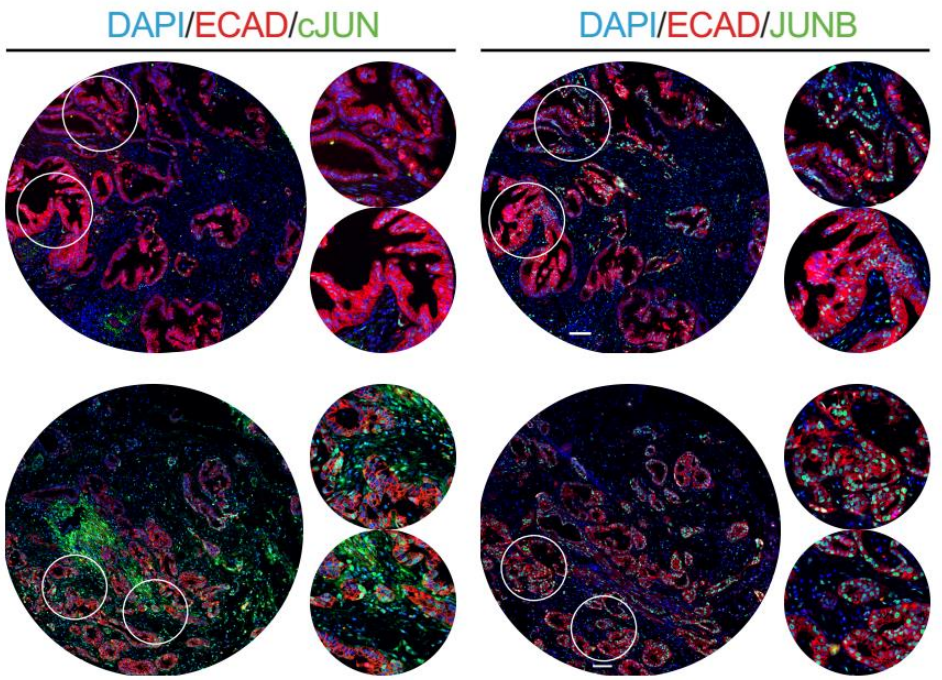

E
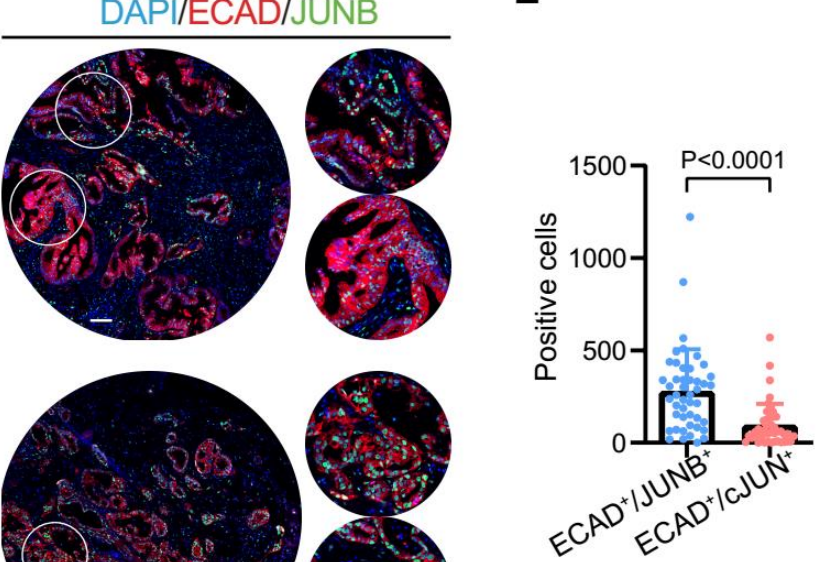

Figure 18. Continued on following page. 


\section{$\mathrm{F}$}

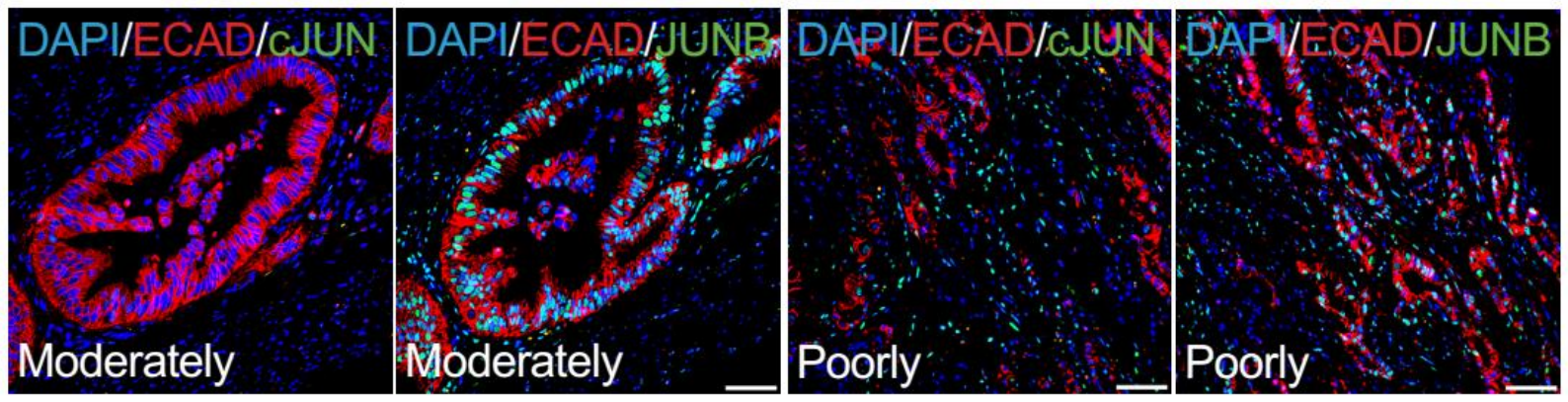

Human PDAC tumors

Figure 18. Subtype-restricted AP1 transcriptional program in PDAC. (A) Bar graph shows the normalized enrichment score and adjusted $P$ values of selected pathways in CLA and BL subtypes based on RNA microarray analysis of 309 PDAC patients (Puleo et al., 2018). (B) Western blot analysis of JUN/AP1 family (CJUN, JUNB and JUND), and ECAD in CLA (CAPAN1 and CAPAN2) and BL (PANC1 and MiaPaCa2) PDAC cells. Actin was used as a loading control. Representative of three independent experiments. (C) Representative IF images of cJUN and JUNB staining in CLA and BL orthotopic tumors. Scale bar: $50 \mu \mathrm{m}$. (D) Representative H\&E (left panel) and IF staining of cJUN/ECAD (middle panel) as well as JUNB/ECAD (right panel) in tissue microarray (TMA) spots of 46 PDAC patients, with identical patients in each row. Scale bar: 100 $\mu \mathrm{m}$. (E) Number of $\mathrm{JUNB}^{+} / \mathrm{ECAD}^{+}$as well as $\mathrm{cJUN}{ }^{+} / \mathrm{ECAD}^{+}$cells in TMA described in (D). Scatter plot shows average value per patient of all evaluated TMA spots (1-3 per patient), with means \pm s.d. as bar graphs. Mann-Whitney test was used to determine the statistical significance $n=46$. $(F)$ Representative IF images of CJUN/ECAD and JUNB/ECAD in moderately differentiated area as well as poorly differentiated primary PDAC tissues. Scale bar: $50 \mu \mathrm{m}$.

\subsubsection{Differential genome binding profile of CLA-JUNB and BL-CJUN}

Since JUNB and cJUN were found to be restricted in the specific PDAC subtype, we sought to explore the underlying molecular mechanism and whether they contributed to PDAC subtype identity. Thus, chromatin immunoprecipitation followed by sequencing (ChIP-seq) for JUNB and cJUN was carried out in CLA and BL cells, respectively. The genome binding profiles of JUNB and cJUN were clearly separated by principal component analysis (Figure 19A). CLA cells could be easily distinguished from BL cells 
by the differential binding profile (Figure 19B). To specify the potential role of cJUN and JUNB in PDAC, the genes linked to regions which were JUNB bound and gained openness as identified by assay for transposase-accessible chromatin followed by sequencing (ATAC-seq) (data not shown) in the CLA (CAPAN1) cells over the BL (PANC1) cells were used for Gene Ontology analysis (GO). Similarly, the genes bound by cJUN and gained openness in the BL PANC1 over the CLA CAPAN1 were selected. This analysis revealed that CAPAN1/JUNB-specific genes were involved in lipid biosynthesis, cell-cell adhesion regulation, and epithelial cell differentiation (Figure 19C). However, PANC1/cJUN-specific genes were correlated with vasculature development, leukocyte migration, and cytokine production (Figure 19D). These data suggest that JUNB- or cJUN-controlled loci are linked to the subtype-specific phenotypic state. 
A

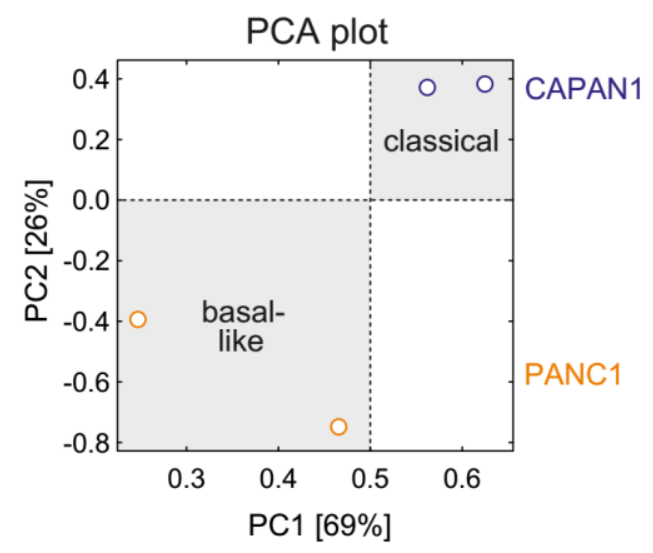

C

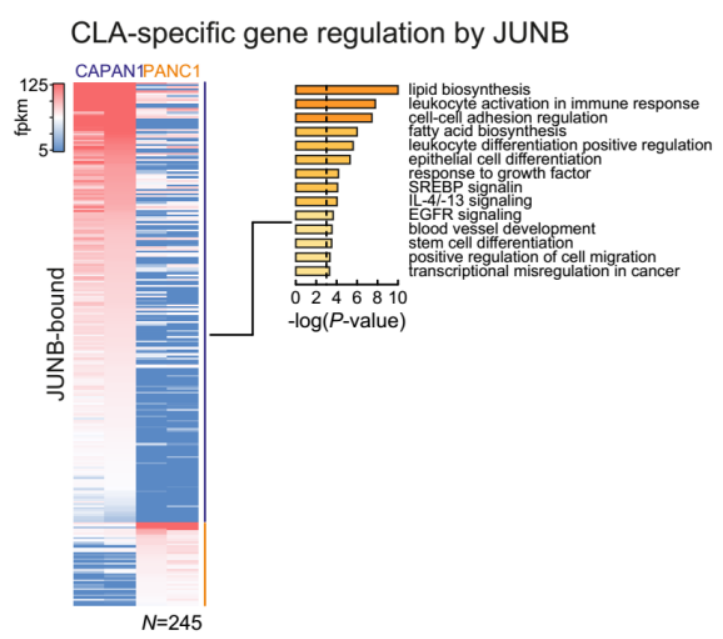

B
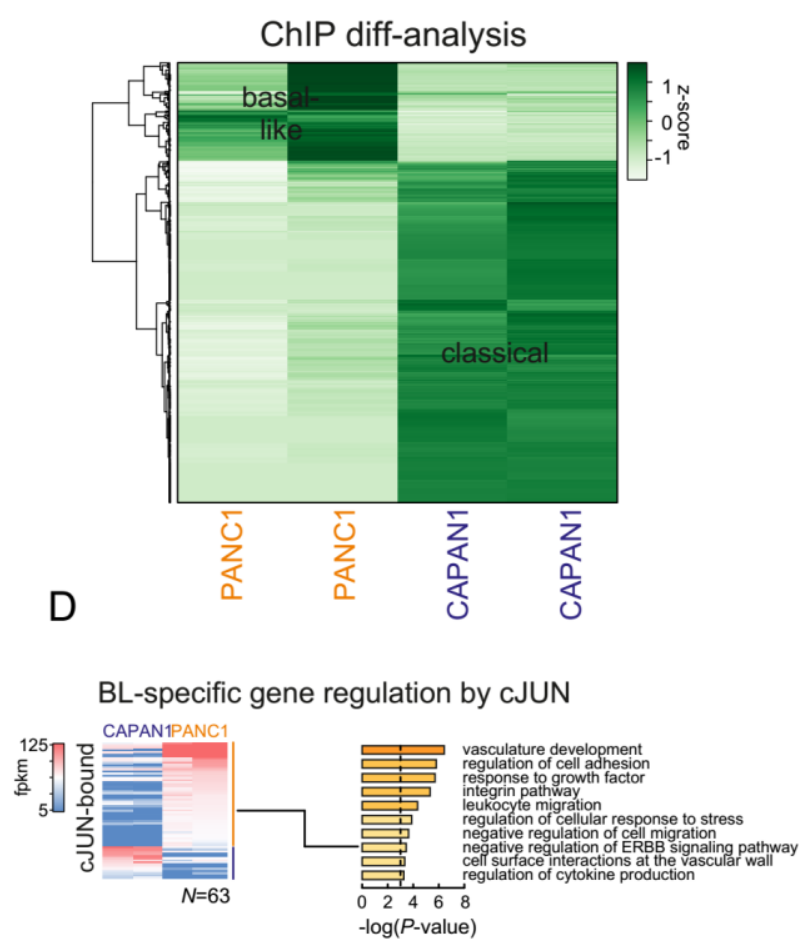

Figure 19. Differential genome binding profile of cJUN and JUNB. (A) Principal component analysis (PCA) of JUNB and cJUN ChIP-seq profiles from CLA (CAPAN1) and BL (PANC1) cell lines. Two biological repeats were included for each cell line. (B) Heatmap of differential binding profile of ChIP-seq data of JUNB and cJUN in the CLA (CAPAN1) and BL (PANC1) cells. (C and D) Heatmaps of JUNB- or cJUN-occupied regions intersected with ATAC-seq peaks in CAPAN1 (E) and PANC1 (F) respectively. Bar plots show gene ontology (GO) terms enriched for the genes assigned to each set of peaks.

In line with the GO analysis, JUNB specifically occupies the promoter and enhancer regions of $C D H 1$ (encodes the ECAD protein). Accordingly, high occupancy of H3K27ac which indicates for activate promoters and enhancers was seen in the CLA CAPAN2 cells 
at the same loci, but not in BL PANC1 cells (Figure 20A). Conversely, cJUN bound the VIM promoter and enhancer loci, accompanied by occupancy of H3K27ac specifically in BL PANC1 cells (Figure 20B). These data suggest the positive regulation of the epithelial marker $C D H 1$ by JUNB, as well as the mesenchymal regulator VIM by cJUN. ChIP-qPCR validated that JUNB indeed bound on the promoter and one of the enhancer regions on CDH1 with strong H3K27ac (Figure 20C and 20D), while cJUN occupied both promoter and enhancer regions of VIM, associated with H3K27ac occupancy (Figure 20E and 20F). These data indicate that JUNB might control cell differentiation via regulation of $\mathrm{CDH} 1$, on the other hand, cJUN may support the BL phenotype through VIM.

A

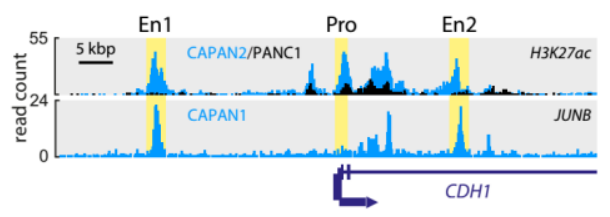

C

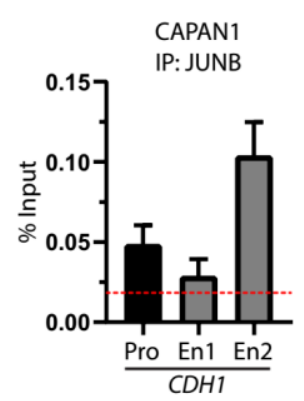

\section{B}

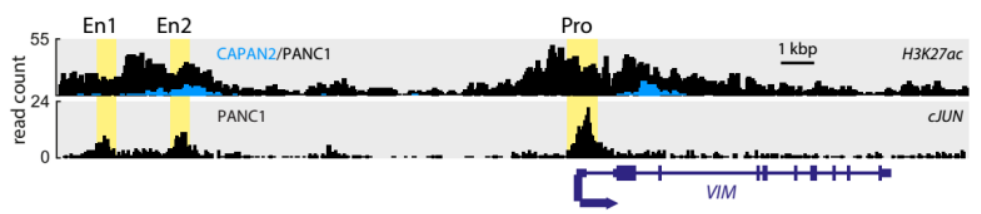

F

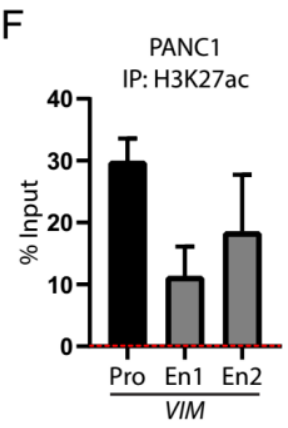

Figure 20. JUNB occupies $C D H 1$ active promoter and enhancer and loci whereas CJUN binds on VIM. Coverage plots of ChIP-seq for JUNB in CLA CAPAN1 cells, as well as H3K27ac in CLA CAPAN2 and BL PANC1 cells at CDH1 locus. For H3K27ac ChIP-seq data, publicly available dataset was utilized (Diaferia et al., 2016). Enhancer (En) and promoter regions (Pro) are indicated. (B) Coverage plots of ChIP-seq for CJUN in BL PANC1 cells, as well as H3K27ac in CLA CAPAN2 and BL PANC1 cells at the VIM locus. Enhancer (En) and promoter regions (Pro) are highlighted. (C and D) qRT-PCR analysis following ChIP of JUNB (C) and H3K27ac (D) on $C D H 1$ promoter and enhancer regions indicated in (A). $n=3$. (E, F) qRT-PCR analysis following ChIP of cJUN (E) and H3K27ac (F) on VIM promoter and enhancer regions indicated in (B). $n=3$. For (C-F), IgG isotype control levels are indicated in red. 


\subsection{3 cJUN overexpression induces invasiveness of CLA PDAC cells}

To explore the functional significance of cJUN in driving a PDAC BL subtype identity, we established retrovirally transduced CLA cells with constitutive cJUN overexpression. Figure 21A confirmed that cJUN was successfully overexpressed in the CLA CAPAN1 cells. Notably, cJUN overexpression led to a dramatic morphological change from wellclustered islands towards a more spindle-like shape (Figure 21B). 3D-transwell invasion assay was conducted to compare the invasiveness between CAPAN1 empty vector (EV) and cJUN overexpressing (CJUN-OE) cells. Interestingly, the number of invading cells was significantly induced following cJUN overexpression (Figure 21C and 21D), suggesting that cJUN promotes an invasive behavior of PDAC cells.
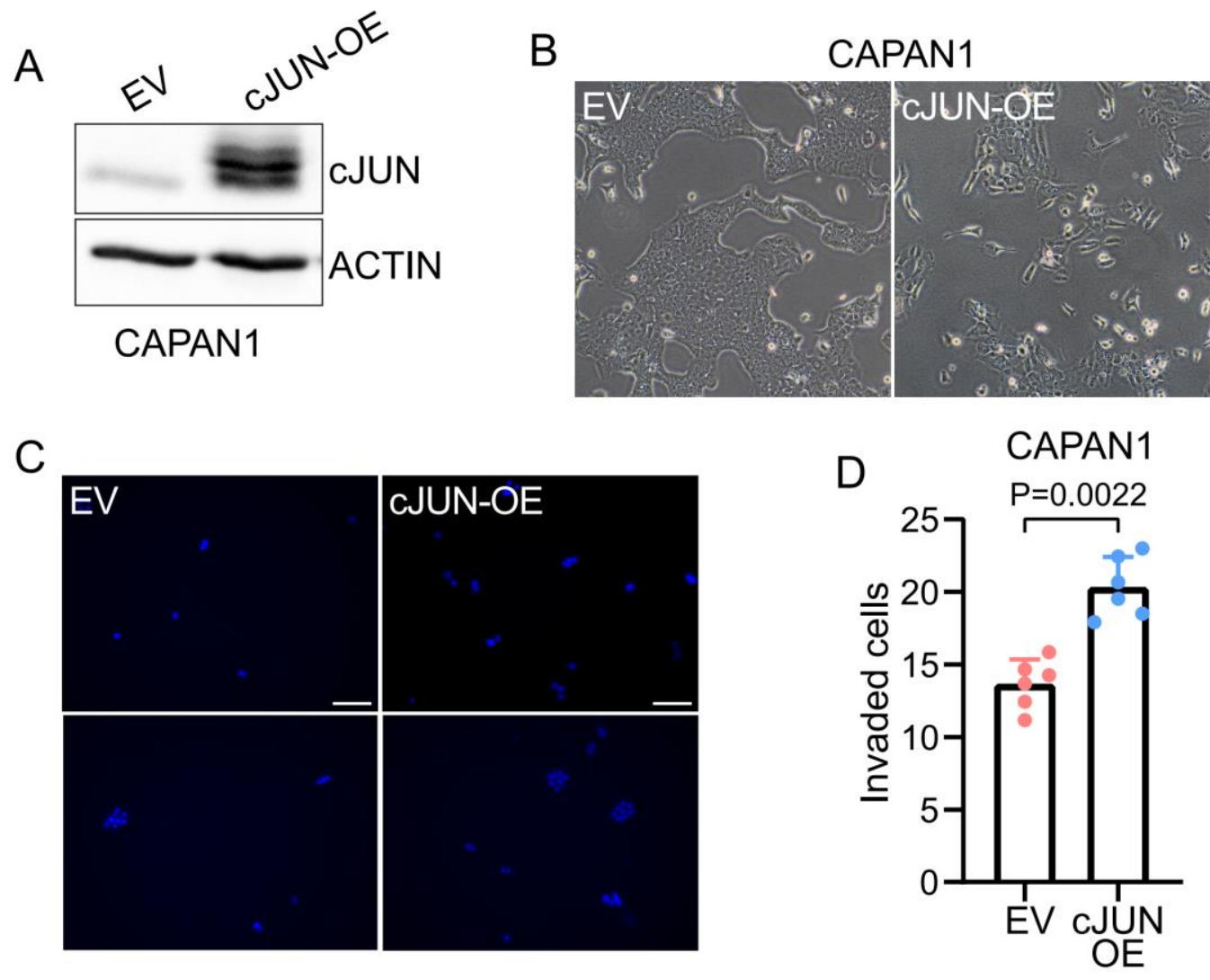

Figure 21. cJUN induces the invasive potential of CLA PDAC cells. (A) CLA CAPAN1 cells were transduced with either an empty vector (EV) or a stable cJUN overexpression (cJUN-OE) construct. Western blot analysis was performed to confirm the efficiency of cJUN overexpression. Actin was used as a loading control. Representative of three independent experiments. (B) Bright- 
field images of (A). (C) 3D-transwell invasion assay of CAPAN1-EV and CAPAN1-cJUN-OE cells. Representative images show DAPI staining of invaded cells. Scale bar: $100 \mu \mathrm{m}$. (D) Quantification of invaded cells of (C). Scatter plot shows average values of count per field of view per individual technical replicate, as well as means \pm s.d. as bar graphs. Mann-Whitney test was used to determine the statistical significance. $n=3$.

Since BL subtype is in general more resistant to chemotherapy treatment, we sought to investigate whether cJUN-OE cells lose sensitivity to chemotherapies. Cell viability of EV and cJUN-OE cells was examined upon gemcitabine, oxaliplatin, and SN38 treatment across a wide range of concentrations as indicated in Figure 22. cJUN-OE cells showed a decreased sensitivity to gemcitabine with $\mathrm{IC}_{50}$ values of $14.6 \mathrm{nM}$ compared to $\mathrm{EV}$ of $10.92 \mathrm{nM}$ (Figure 22A). A similar effect was seen following the treatment with oxaliplatin (cJUN-OE IC $50=1458 \mathrm{nM}$ vs EV IC $50=744.5 \mathrm{nM}$ ) (Figure 22B) as well as the active irinotecan metabolite SN38 (cJUN-OE IC $50=4.86 \mathrm{nM}$ vs EV IC $50=1.63 \mathrm{nM}$ ) (Figure 22C). These data suggest that cJUN contributes to chemoresistance in PDAC cell lines.
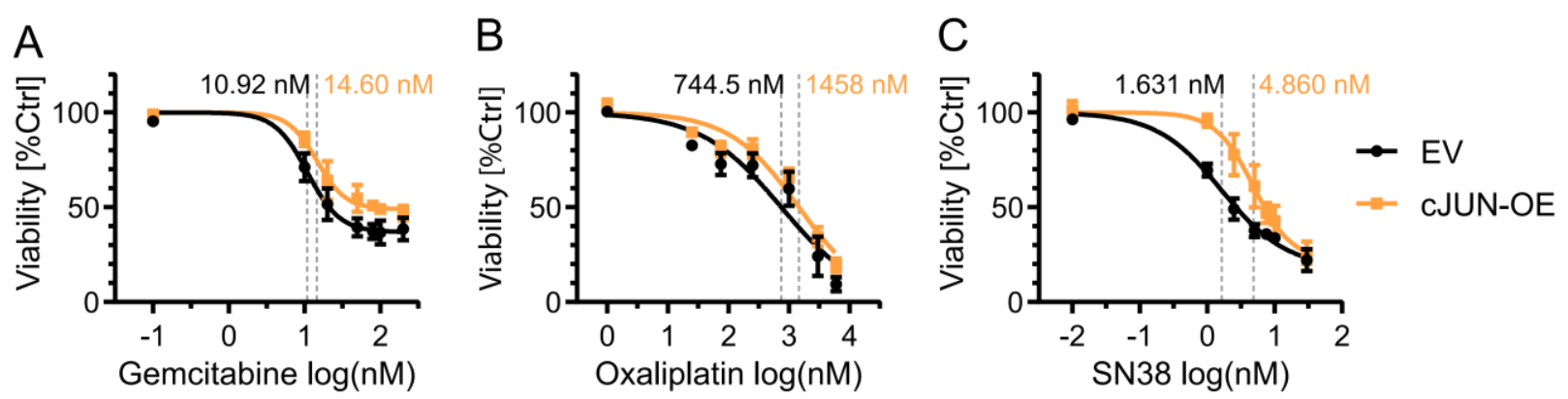

Figure 22. cJUN overexpression contributes to resistance to chemotherapeutics. CAPAN1EV and cJUN-OE cells were seeded in 96-well plates and treated with gemcitabine, oxaliplatin, and SN38 at different concentrations indicated in the figure for $72 \mathrm{~h}$. CellTiter-Glo was utilized to examine the cell viability. Dose response curves show cell viability of EV (black) and CJUN-OE (yellow) cells upon gemcitabine $(A)$, oxaliplatin $(B)$, and $S N 38(C) . I_{50}$ values for each drug are indicated $n=3$. 


\subsection{4 cJUN-CCL2 regulation in BL PDAC cells}

Since the BL subtype has high levels of CCL2 as well as cJUN/AP1, which is known as an inflammatory transcriptional factor, we hypothesized that cJUN may regulate CCL2 to recruit macrophages. Thus, we took advantage of our cJUN ChIP-seq data in BL PANC1 cells and visualized ChIP-seq tracks on Integrative Genomics Viewer (IGV). A significant enrichment of cJUN on both promoter and enhancer regions of CCL2 could be observed (Figure 23A), indicating that CJUN binds on the CCL2 gene in PDAC. For further validation, we performed individual ChIP-qPCR analysis for cJUN and H3K27ac in PANC1 cells with primers designed on these regions, as indicated in Figure 23A. Consistently, we found a significant occupancy of cJUN on the CCL2 promoter as well as enhancer accompanied with strong H3K27ac (Figure 23B and 23C), suggesting that cJUN might positively regulate CCL2 expression.

A

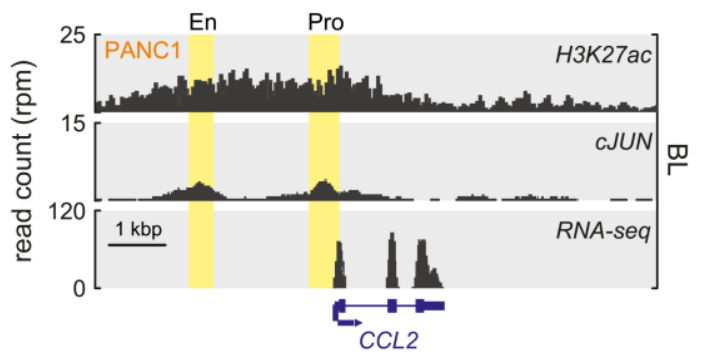

B

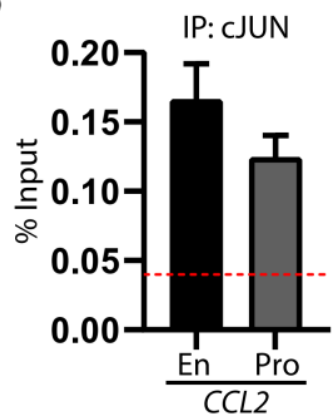

C

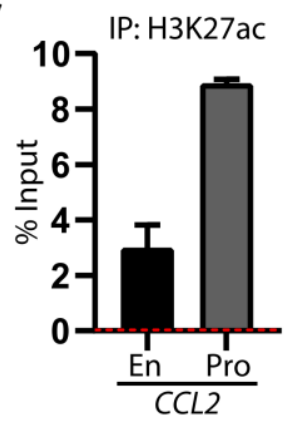

Figure 23. cJUN occupies active promoter and enhancer regions of CCL2 in BL cells. (A) Coverage plots of ChIP-seq for cJUN and H3K27ac, as well as RNA-seq in BL PANC1 cells at CCL2 locus. For H3K27ac ChIP-seq and RNA-seq data in PANC1 cells, a publicly available dataset was utilized (Diaferia et al., 2016). Enhancer (En) and promoter regions (Pro) are highlighted. (B and C) qRT-PCR analysis following ChIP of cJUN (B) and H3K27ac (C) on CCL2 promoter and enhancer regions indicated in $(A) n=2$.

\subsubsection{Loss of cJUN leads to reduction of CCL2}

To validate the regulation of CJUN on CCL2, we subjected BL PANC1 cells to cJUNtargeting siRNA transfection and tested cJUN expression on Western blot (Figure 24A). 
siRNA \#1 showed higher knockdown efficiency for cJUN. Therefore, it was used in all following experiments (Figure 24A). qRT-PCR confirmed siRNA-mediated knockdown on mRNA level. We observed that CCL2 mRNA expression was significantly decreased upon cJUN silencing (Figure 24B). cJUN-dependent expression of CCL2 was further validated at the protein level by Western blot analysis using the established BL cell lines PANC1 and PT45P1 (Figure 24C and 24D). To test whether this effect was reproducible in the cells isolated from patient-derived xenografts (PDX), cJUN-high cell lines GCDX5 and GCDX57 were utilized for cJUN silencing. Consistently, CCL2 was considerably reduced following loss of cJUN (Figure 24E and 24F). These data strongly suggest that cJUN positively regulates CCL2 in BL PDAC cells.

A

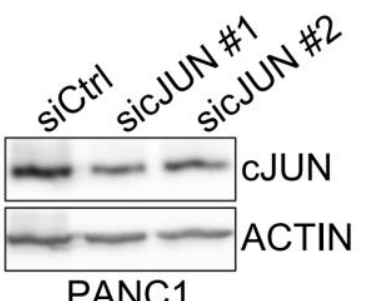

PANC1
B

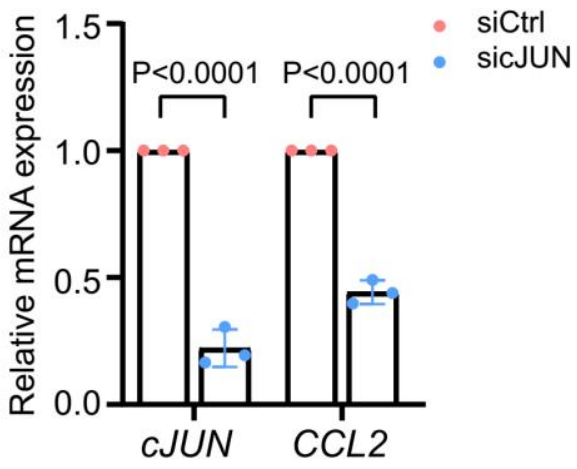

C

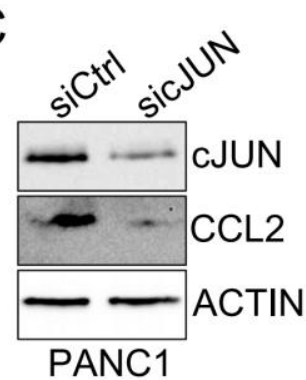

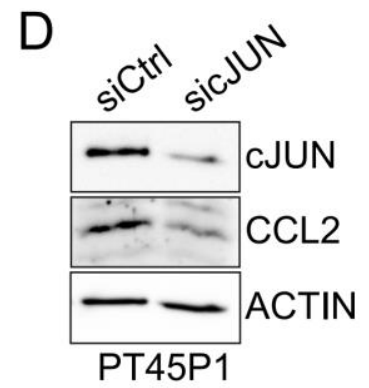

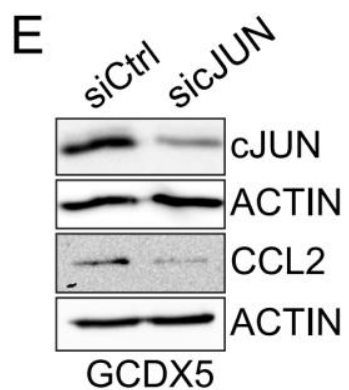

F

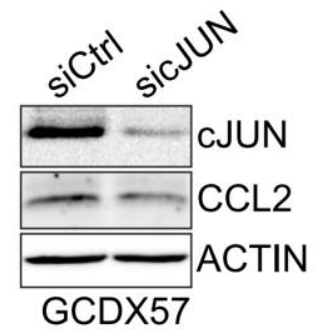

Figure 24. Effect of cJUN depletion on CCL2. (A) BL PANC1 cells were transfected with either siCtrl or different sicJUN sequences for $48 \mathrm{~h}$. Cell lysates were subjected to Western blot analysis to test the efficiency of cJUN knockdown. Representative of three independent experiments. (B) qPCR analysis was performed for CJUN and CCL2 in BL PANC1 cells upon $48 \mathrm{~h}$ transfection of either siCtrl or sicJUN. Bar graphs show means \pm s.d. Unpaired student's t-test was used for statistical significance. $n=3$. (C-F) Western blot analysis of cJUN and CCL2 following cJUN silencing in established BL cells PANC1 (C), PT45P1 (D), as well as cell lines isolated from 
patient-derived xenograft GCDX5 (E), and GCDX57 (F). Actin was used as loading control. Representative of three independent experiments.

\subsubsection{TNFa upregulates cJUN and CCL2 expression}

It is evident that TNFa is secreted from the TME and contributes to tumor progression as well as maintenance of the BL phenotypic state. Thus, we reasoned that TNFa might activate the inflammatory program in tumor cells to constitutively produce cytokines such as CCL2, in turn forming a positive loop for the recruitment of immune cells. To validate our hypothesis, we treated CLA (CAPAN1 and CAPAN2) as well as PDX-derived (GCDX62) cell lines with TNFa (10ng/ $\mathrm{LL})$ in a time dependent manner, as shown in Figure 25. Following the treatment with TNFa, expressions of cJUN as well as CCL2 were markedly induced, with robust effects seen at $8 \mathrm{~h}$ treatment (Figure 25).

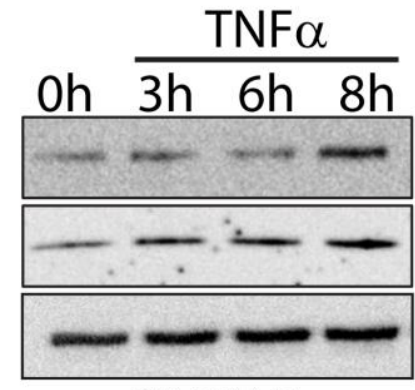

CAPAN1

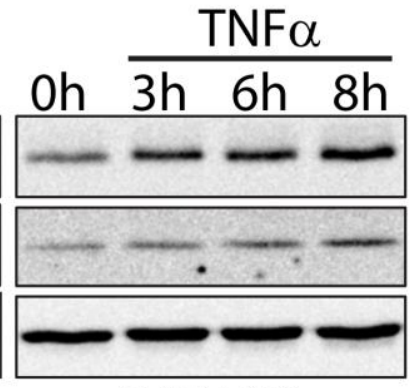

CAPAN2

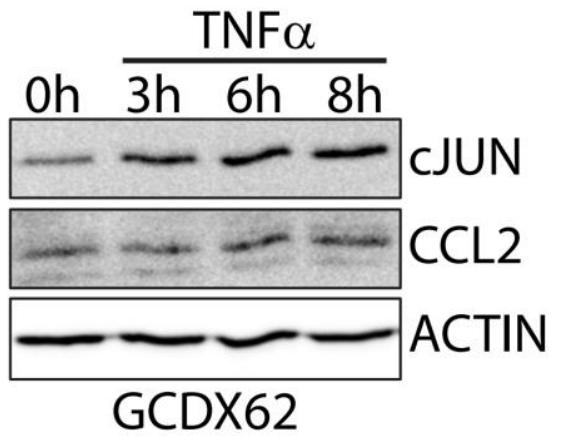

Figure 25. Exogenous TNFa treatment induces expression of cJUN and CCL2 in CLA cell lines. Western blot analysis of cJUN and CCL2 following TNFa treatment ( $10 \mathrm{ng} / \mathrm{mL})$ for $3 \mathrm{~h}, 6 \mathrm{~h}$, and $8 \mathrm{~h}$ in CAPAN1, CAPAN2, and GCDX62. Actin was used as loading control. Representative of three independent experiments.

For further validation, we engineered CLA PDAC cell lines to stably overexpress TNFa. Cells were transduced with either empty vector (EV) or lentiviral construct which carries a TNFa-mCherry insert. The expression of TNFa in these cells was confirmed by flow cytometry analysis (Figure 26A). CAPAN1- and CAPAN2-TNFa-OE cell lines displayed a significant induction of cJUN and CCL2 compared to their respective EV control (Figure 
26B and 26C), along with a phenotypic switch from well-clustered toward a more spindlelike mesenchymal morphology in 2D culture (Figure 26D). This confirms that TNFa activates the expression of CJUN and CCL2 and promotes a more aggressive BL phenotype.

A

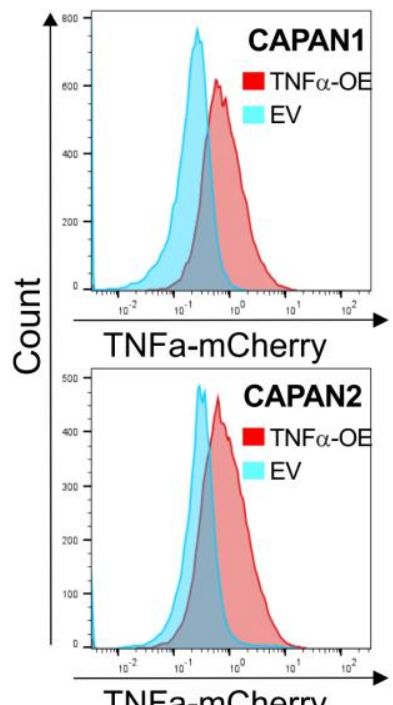

B
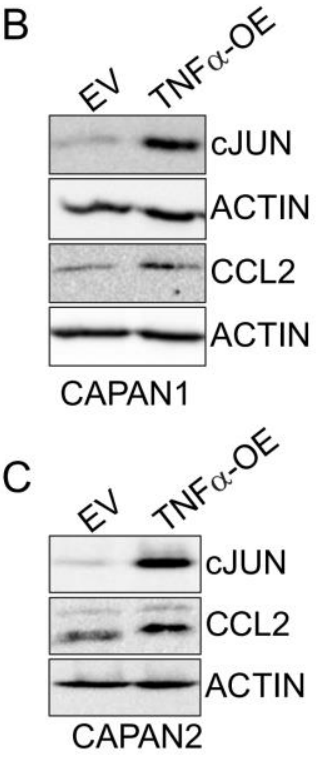

$\mathrm{D}$

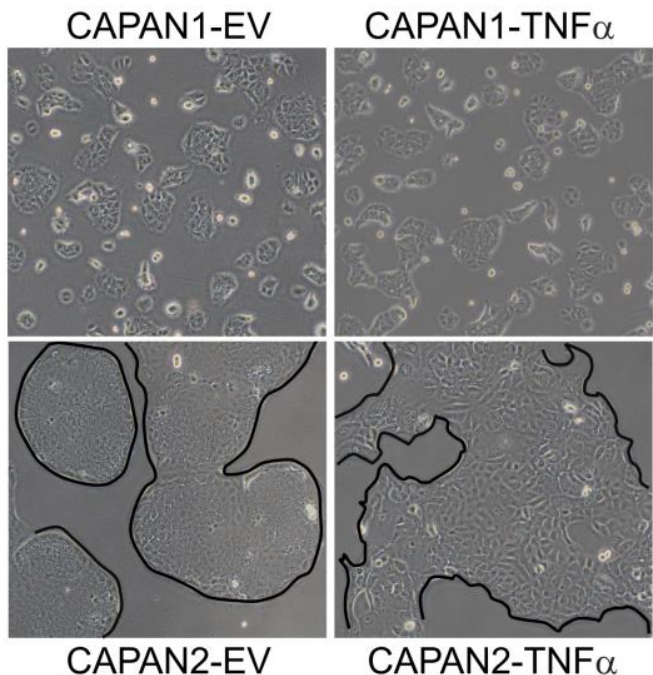

Figure 26. TNFa overexpression leads to induction of cJUN and CCL2. (A) Flow cytometric analysis of TNF $\alpha$ expression in CAPAN1-EV, CAPAN1-TNFa-OE, CAPAN2-EV, CAPAN2-TNFaOE cells. $561 \mathrm{~nm}$ laser was used to detect TNFa-mCherry expression. Count of gated cells is shown against fluorescence intensity, which correlates to TNFa expression. $n=3$. ( $B$ and $C$ ) Western blot analysis of cJUN and CCL2 in CAPAN1-TNFa-OE cells (B) as well as CAPAN2TNFa-OE cells (C). Actin was used as a loading control. Representative of three independent experiments. (D) Representative bright field images show morphology of CAPAN1-EV, CAPAN2EV and their corresponding TNFa overexpression cell lines.

\subsubsection{TNFa induces CCL2 expression via cJUN in PDAC}

To determine whether cJUN was essential for TNFa-induced CCL2 expression, we utilized TNFa-OE cell lines and subjected them to cJUN siRNA transfection to test whether CCL2 expression is altered. Indeed, CJUN and CCL2 levels were significantly induced in both TNFa-OE cell lines. However, following siRNA-mediated depletion of 
cJUN, the induction of CCL2 was alleviated (Figure 27A and 27B). This data suggests that TNFa induces CCL2 expression through a cJUN-dependent mechanism.

A

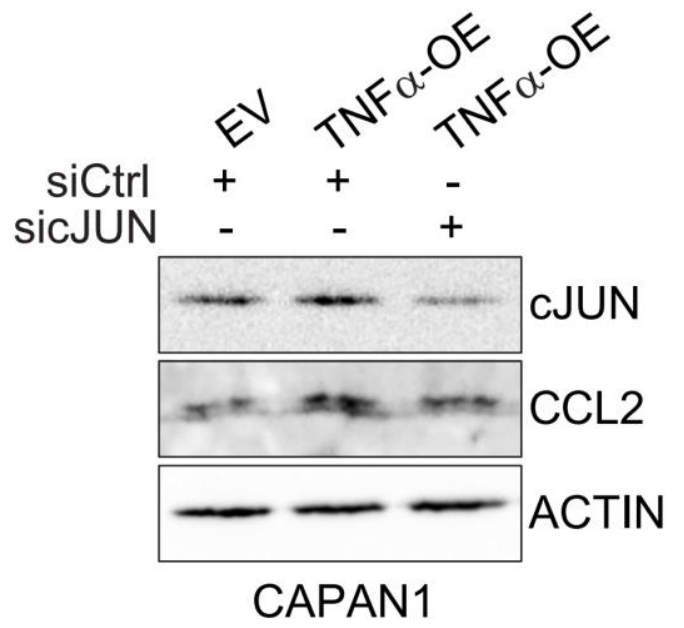

$\mathrm{B}$

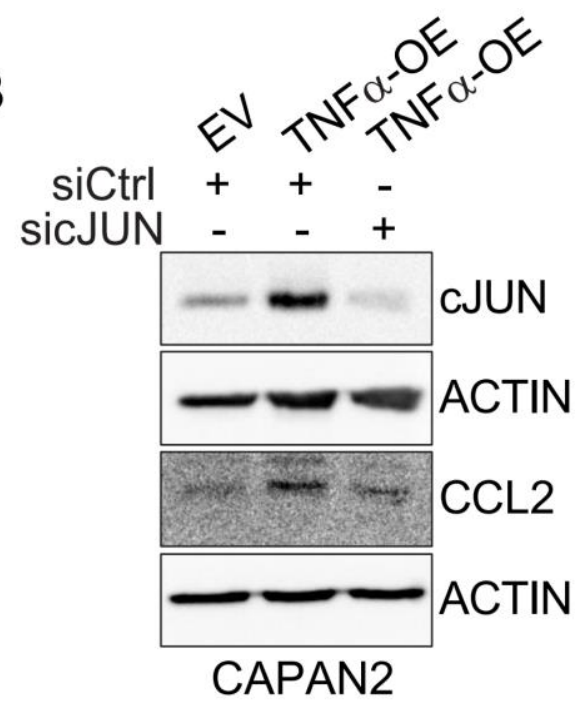

Figure 27. TNFa induced CCL2 is dependent on cJUN. (A and B) CAPAN1-TNFa-OE (A) and CAPAN2-TNFa-OE (B) cells were transfected with either siCtrl or sicJUN for $48 \mathrm{~h}$. Cell lysates were subjected to Western blot analysis to test the expression of CJUN, CCL2. Actin was used as a loading control. Representative of three independent experiments.

\subsection{TNFa monotherapy does not improve overall survival}

So far, we identified a positive feed forward loop that $\mathrm{TNFa}^{+}$producing macrophages induce cJUN-CCL2 signaling in tumor cells, further recruiting macrophages to sustain the $B L$ subtype identity. Therefore, we investigated whether inhibition of TNFa alone was able to abrogate this feed forward loop and support a favorable prognosis. A syngeneic KPC mouse model was established by implanting KPCbl6 cells into C57BL/6J mice (Figure 28A). After tumor development, mice were administered anti-TNFa antibodies or IgG isotype control via i.p. injection. Survival was recorded when mice reached end point criteria, for example $20 \%$ bodyweight lost, unkempt hair, looking dull or abnormal posture. However, the survival rate of $\lg G$ and anti-TNFa-treated mice did not differ significantly (Figure 28B). 
A

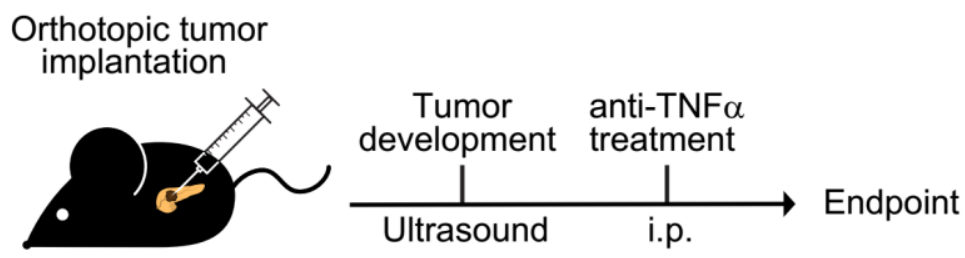

KPCbl6

B

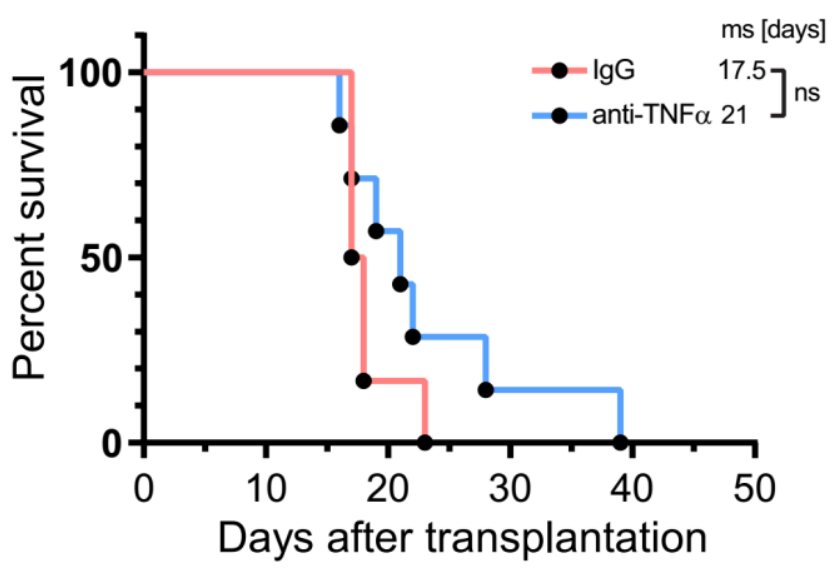

Figure 28. Inhibition of TNFa failed to improve the survival of syngeneic KPC mice. (A) illustration of experimental settings. 10-weeks old male C57BL/6J mice were orthotopically transplanted with $3.5 \times 10^{4} \mathrm{KPCb} 6$ cells. Tumor development was ensured by ultrasound. Antimouse TNF $\alpha$ antibody (10 $\mu \mathrm{g} / \mathrm{g}$ body weight) was applied three times per week. (B) Kaplan-Meier plot indicating survival of IgG (red) and anti-TNFa-treated (blue) syngeneic KPC mice. Median survival (ms) as well as significance (as per log-rank test) are indicated.

H\&E staining was performed in the $\lg G$ and TNFa-treated syngeneic KPC tumors. Tumor histology showed poor differentiation in general; however, we did observe any difference between control and treatment groups (Figure 29A). Histological quantification of welldifferentiated tumors confirmed that there was no difference in well differentiated area between IgG and TNFa-treated tumors (Figure 29B). Additionally, blocking TNFa did not reduce the recruitment of $C D 45^{+}$cells (Figure 29C and 29D). These data suggest that TNFa inhibition is not an ideal therapeutic option. 
A

B

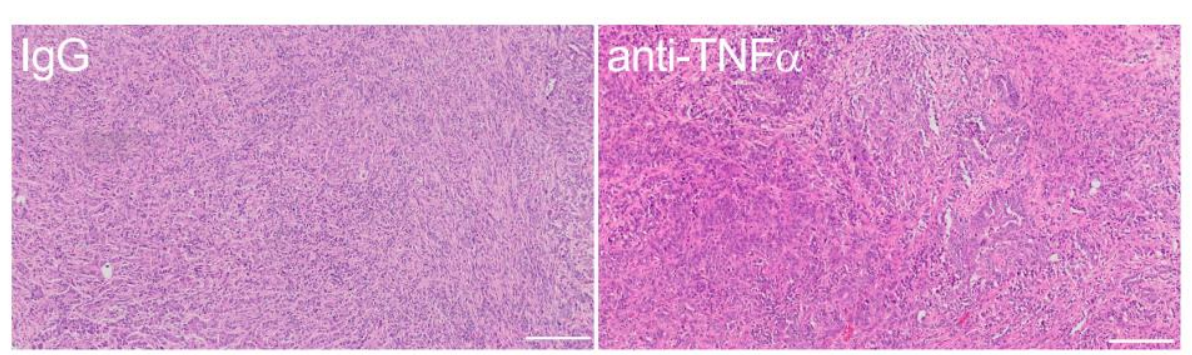

Orthotopic implantation (KPCbl6)

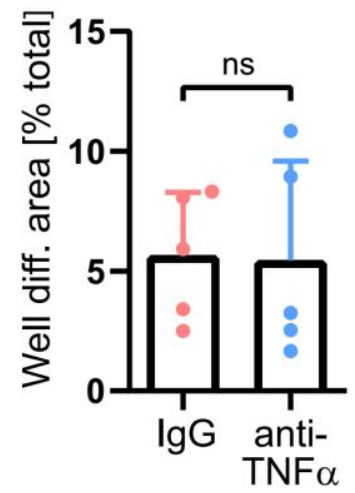

C
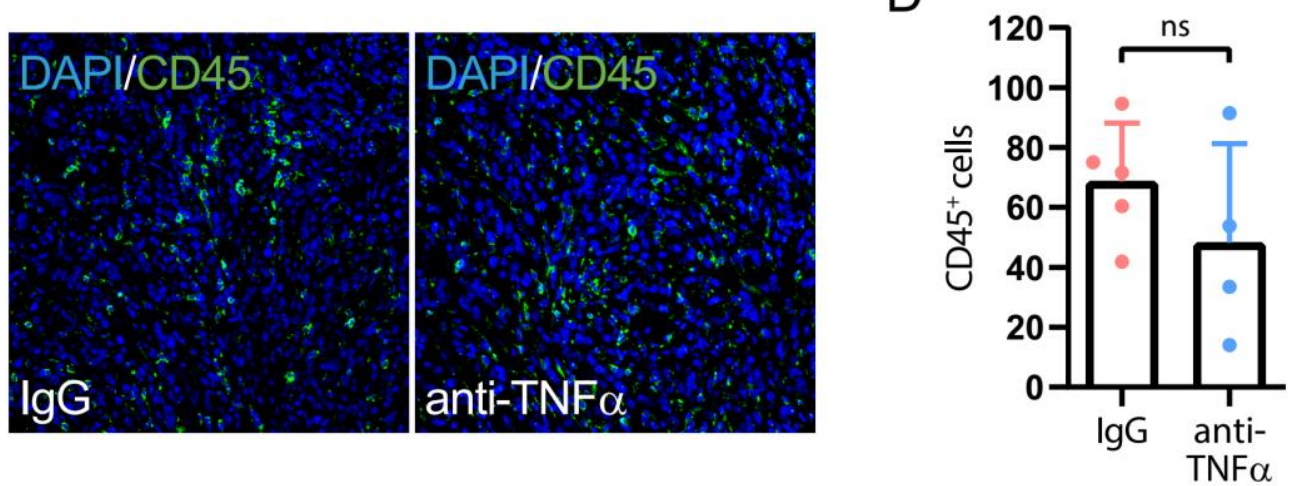

Figure 29. Inhibition of TNFa did not alter tumor differentiation or infiltration of CD45+ cells. (A) Representative tile scan images of $\mathrm{H} \& \mathrm{E}$ staining in IgG and anti-TNFa-treated syngeneic KPC tumors. Scale bar: $200 \mu \mathrm{m}$. (B) Quantification of well differentiated tumor areas in (A). Scatter plots show well differentiated tumor area as percentage of total tumor area per animal, as well as means \pm s.d. as bar graphs. Mann-Whitney test was used for statistical analysis. $n=5$ in lgG; $n=5$ in anti-TNFa. (C) Representative images of IF staining for CD45 in (A). (D) Quantification of CD45 cells in (C). Scatter plots show average values per animal of counts per field of view as well as means \pm s.d. as bar graphs. Mann-Whitney test was used for statistical analysis. $n=5$ in $\lg$; $n=4$ in anti-TNFa.

\subsection{BRD4 controls cJUN expression via enhancer regulation}

Since it is not feasible to block the cJUN-CCL2 signaling axis by TNFa inhibition, and there is no therapeutic drug available for specifically targeting TF cJUN, we sought to find 
a potential chromatin regulator which may control cJUN expression in the BL subtype. Thus, we utilized a publicly available ChIP-seq dataset in PDAC cells (Diaferia et al., 2016) and examined differential occupancy of the active transcription mark Histone 3 lysine 4 trimethylation ( $\mathrm{H} 3 \mathrm{~K} 4 \mathrm{me} 3)$ as well as $\mathrm{H} 3 \mathrm{~K} 27 \mathrm{ac}$ between CLA and BL cell lines by the Enrichr tool (Chen et al., 2013; Kuleshov et al., 2016). Strikingly, we found not only cJUN binding regions to be significantly enriched (defined by H3K4me3 and H3K27ac), but also the bromodomain co-regulator BRD4 in the BL-specific active genomic regions (Figure 30).

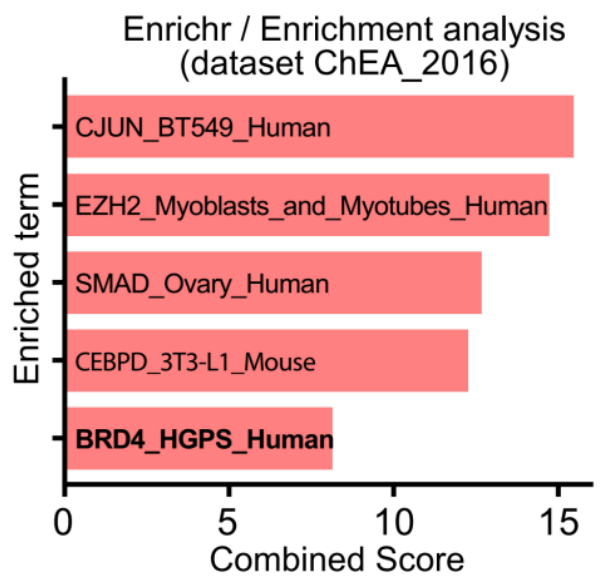

Figure 30. Enrichment score of cJUN and BRD4. Publicly available ChIP-seq dataset (Diaferia et al., 2016) was reanalyzed by F. Wegwitz (Department of Gynecology and Obstetrics, UMG, Germany) for the differential occupancy of H3K27ac and H3K4me3 (>2 fold change) between the BL and CLA PDAC cells using the Enrichr tool (Chen et al., 2013; Kuleshov et al., 2016).

To validate whether there was an association between cJUN and BRD4, we made use of a published dataset of BRD4 ChIP-seq in acute myeloid leukemia (AML) with DMSO and JQ1 treatment (Bhagwat et al., 2016). JQ1 is a small-molecule inhibitor for bromodomain and extra-terminal domain (BET) family with high affinity towards BRD4 (Filippakopoulos et al., 2010). This data revealed a significant occupancy of BRD4 on cJUN enhancer regions. ChIP-seq data of H3K27ac as well as enhancer marker Histone 3 lysine 4 monomethylation (H3K4me1) also displayed a strong enrichment at cJUN enhancer loci However, the enrichment was reduced upon JQ1 treatment (Figure 31A). Next, we 
performed individual ChIP-qPCR experiments for BRD4 to confirm binding over the putative enhancer regions of cJUN. Indeed, these three loci were found to be strongly occupied by BRD4. As predicted, BRD4 lost its occupancies on these sites following $8 \mathrm{~h}$ JQ1 treatment (Figure 31B).

A
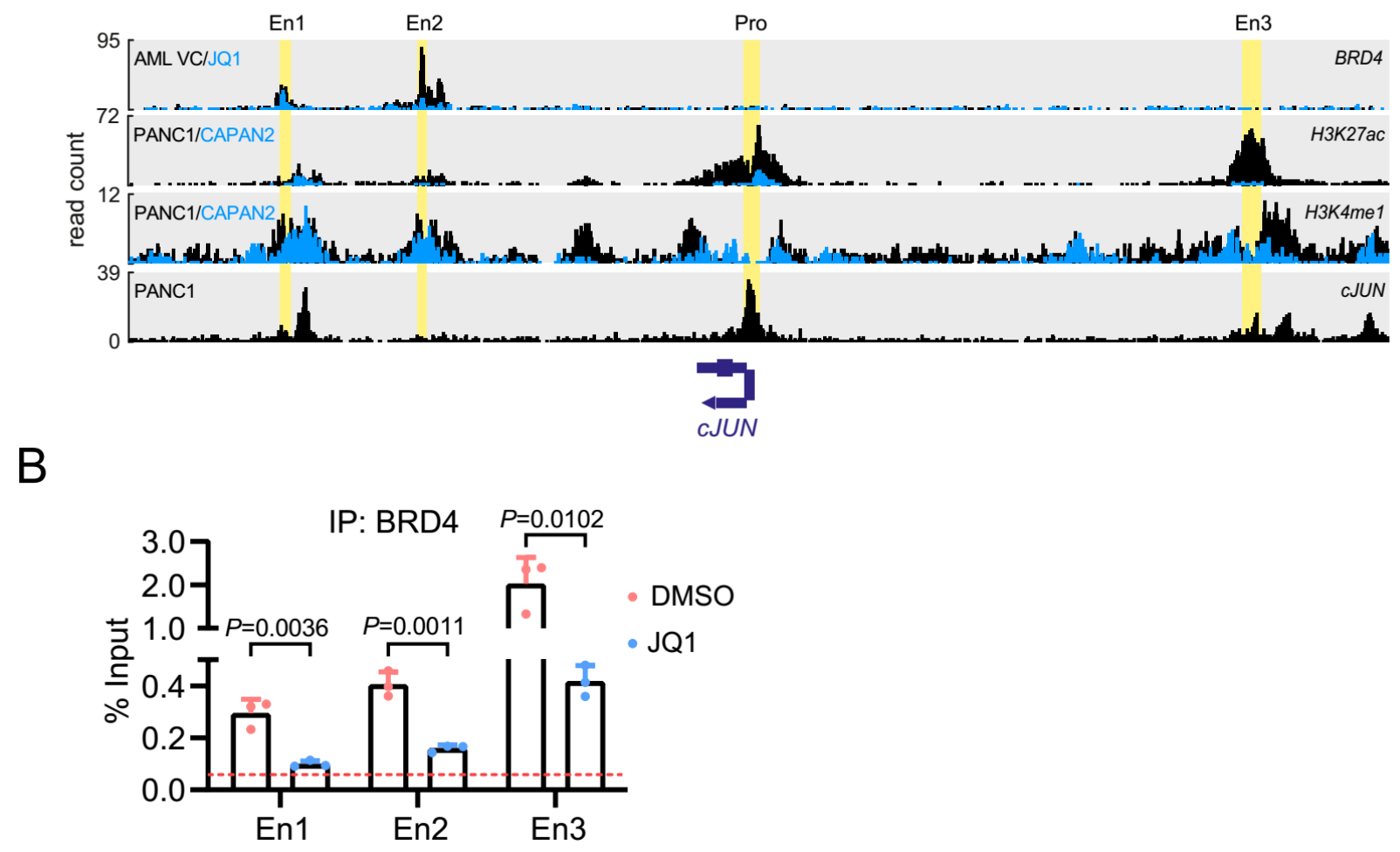

Figure 31. BRD4 occupies cJUN enhancer loci. (A) Coverage of BRD4 ChIP-seq in acute myeloid leukemia (AML) in vehicle control (VC; black) and JQ1-treated (blue) conditions (Bhagwat et al., 2016), as well as H3K27ac and H3K4me1 ChIP-seq in CLA cells (CAPAN2; blue) and BL cells (PANC1; black) (Diaferia et al., 2016) at the cJUN gene locus. (B) ChIP for BRD4 was performed in BL PANC1 cells upon DMSO and JQ1 treatment for $8 \mathrm{~h}$ followed by qRT-PCR analysis. Red dashed line indicates average $\operatorname{lgG}$ isotype control. Bar graphs show means \pm s.d. Mann-Whitney test was used for statistical analysis. $n=3$.

To validate the ChIP-qPCR results, which implies a regulation of cJUN by BRD4, we examined the expression of cJUN upon BRD4 depletion in BL cell lines. First, PANC1 cells were transfected with two different siRNAs to test the knockdown efficiency (Figure 
32A). Accordingly, siBRD4 \#1 was chosen to perform further analysis. Next, BL cell lines were tested for cJUN expression upon BRD4 silencing. We observed a significant reduction of cJUN following BRD4 silencing in both PANC1 and MiPaCa2 cell lines (Figure 32B), suggesting that BRD4 regulates cJUN expression.

A

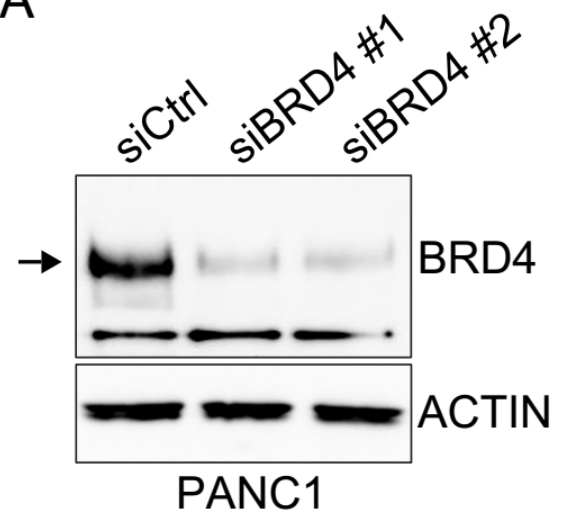

B

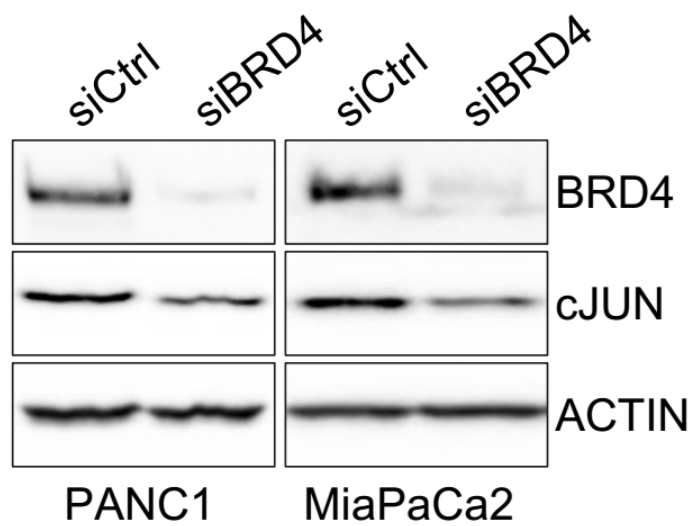

Figure 32. BRD4 positively regulates cJUN in BL cells. (A) Western blot analysis for BRD4 in BL PANC1 cells transfected with either siCtrl or siRNAs targeting BRD4 for $48 \mathrm{~h}$. (B) Western blot analysis of BRD4 and cJUN in siBRD4 or siCtrl transfected BL PANC1 and MiaPaCa2 cells. Actin was used as loading control. Representative of three independent experiments.

\subsection{Pharmacological inhibition of the BRD4-CJUN-CCL2 axis supports a favorable prognosis in preclinical mouse model}

To elucidate the potential of pharmacological inhibition of BRD4, BL PANC1 cells were subjected to JQ1 treatment at different concentrations. As expected from siBRD4 experiments, JQ1 treatment led to a significant downregulation of cJUN at a concentration of $500 \mathrm{nM}$ in BL PANC1 cells (Figure 33). 


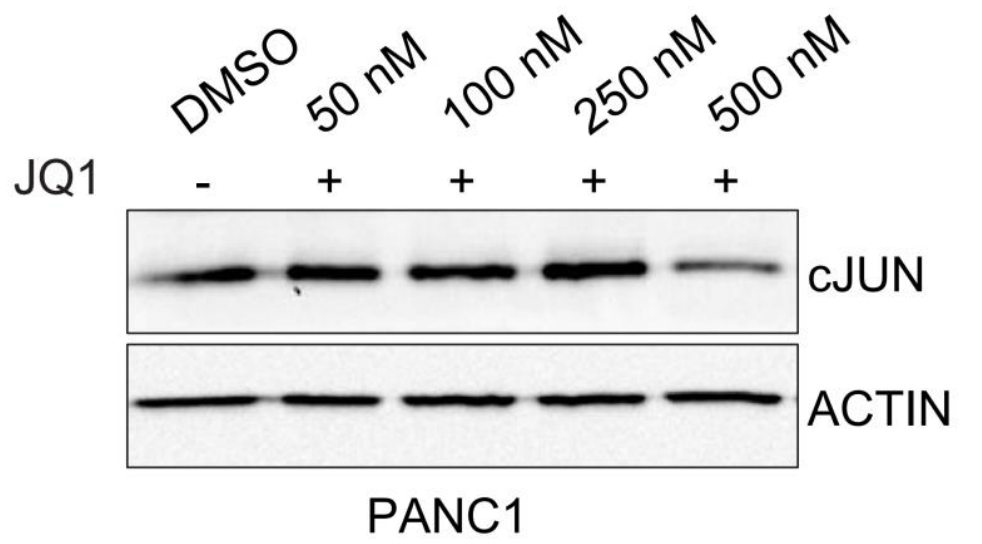

Figure 33. JQ1 treatment leads to reduction of cJUN. PANC1 cells were treated with JQ1 at indicated concentrations. After $24 \mathrm{~h}$, cells were harvest and subjected for Western blot analysis for cJUN. Actin was used as loading control. Representative of three independent experiments.

To investigate the therapeutic effect of JQ1 inhibition on the CJUN-CCL2 signaling axis in vivo, we established a syngeneic orthotopic KPC mouse model using tumor cells derived from KPC mice implanted in 10-week old male C57BL/6J mice (Figure 34A). Tumor growth was detected by sonography on day 10 post-transplantation. After tumor development, mice were treated with DMSO or JQ1 (50 mg/kg bodyweight) daily over three weeks. Survival data was recorded when mice reached end point criteria, as mentioned previously. Under DMSO control treatment, mice only survived for 18 days (median survival). However, JQ1 treatment significantly prolonged median survival to 29 days, thereby supporting a favorable prognosis in the syngeneic KPC mice (Figure 34B). 
A

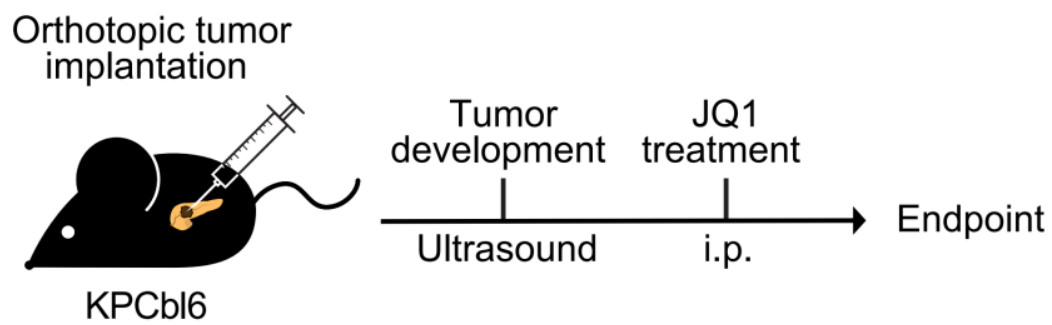

B

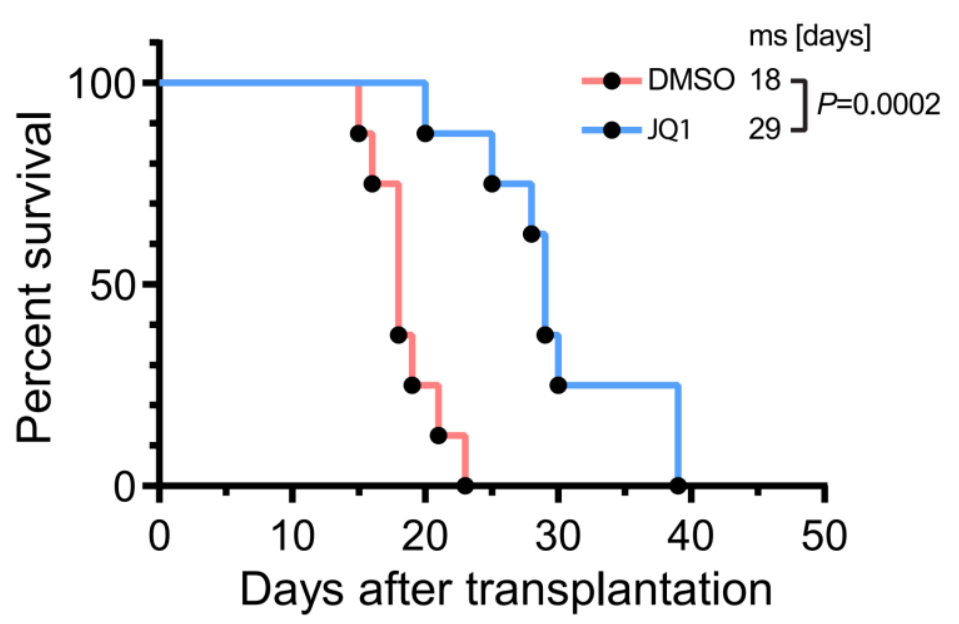

Figure 34. JQ1 resulted in a significant improvement of survival. (A) Diagram of experimental settings to investigate the therapeutic effect of JQ1 in vivo. 10-weeks old male C57BL/6J mice were orthotopically implanted with $3.5 \times 10^{4} \mathrm{KPC}$ tumor cells derived from KPC mice. After 10 days of transplantation, (B) Ultrasound was performed to detect tumor growth. Mice were administrated with JQ1 (50 mg/kg bodyweight) and DMSO control daily. (B) Kaplan-Meier plot indicating survival of DMSO (red) and JQ1-treated (blue) syngeneic KPC mice. Median survival (ms) as well as significance (as per log-rank test) are indicated.

In order to evaluate the potential changes of tumor histology following JQ1 treatment, H\&E staining was performed in the DMSO- and JQ1-treated syngeneic KPC tumors followed by whole tissue tile image scans (Figure 35A). Of note, PDAC tumors derived from syngeneic KPC mice exhibit BL/poorly differentiated histology. Notably, JQ1-treated tumors were observed to gain a more differentiated phenotype, which significantly recapitulates a CLA phenotypic state. The proportion of well differentiated tumor area was considerably induced in the JQ1-treated tumors over DMSO control (Figure 35B). 
A

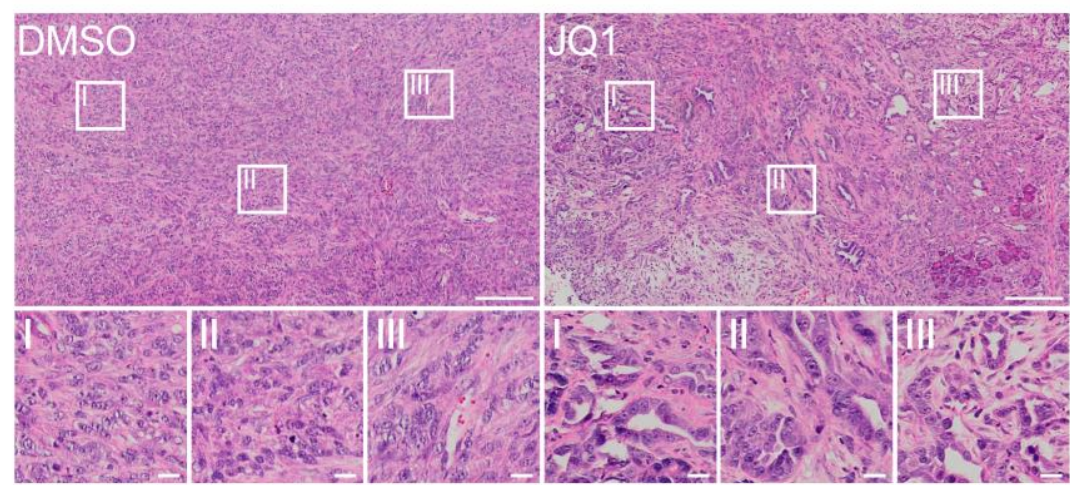

Orthotopic implantation (KPCbl6)
B

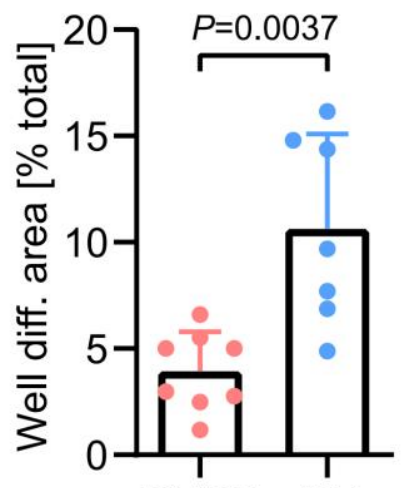

DMSO JQ1

Figure 35. JQ1 treatment restrains tumor dedifferentiation. (A) Representative images of H\&E staining in pancreatic tumor tissues derived from DMSO control and JQ1-treated syngeneic KPC model. I-III depict higher magnification images. Scale bar: representative images (top): $200 \mu \mathrm{m}$; higher magnification images (bottom): $10 \mu \mathrm{m}$. (B) Scatter plot showing quantification of well differentiated tumor area as percentage of the total tumor area in DMSO- and JQ1-treated mice, as well as means $\pm s$.d. as bar graphs. $n=8$, DMSO; $n=7$, JQ1. Mann-Whitney test was used for statistical analysis.

Since a phenotypic switch could be seen in the JQ1 treated tumor tissues, we investigated how tumor cells changed their cellular plasticity. Firstly, VIM expression was investigated by performing IF staining in the DMSO- and JQ1-treated tumors. A significant reduction of VIM with the application of JQ1 was observed (Figure 36A and 36B), which could partially explain the reduced poorly differentiated phenotype in the JQ1-treated tumors. To further validate whether the JQ1-triggered phenotypic switch was mediated through cJUN, the expression of TNFa and cJUN as well as CCL2 were probed in the syngeneic KPC tumors. It showed that cJUN was dramatically reduced in the treatment of JQ1 (Figure 36A and 36C), concomitant with a reduction in the expression TNFa and CCL2 (Figure 36A, 36D and 36E), resulting a reduction of the infiltration of $\mathrm{CD} 45^{+}$cells (Figure 36A and 36F). Taken together, these data suggest that inhibition of BRD4 by JQ1 abrogates the cJUN-CCL2-TNFa regulatory axis in neoplastic cells, which further inhibits 
the infiltration of $\mathrm{TNFa}^{+}$immune cells in the TME, and thus, supports a favorable prognosis in PDAC.

A

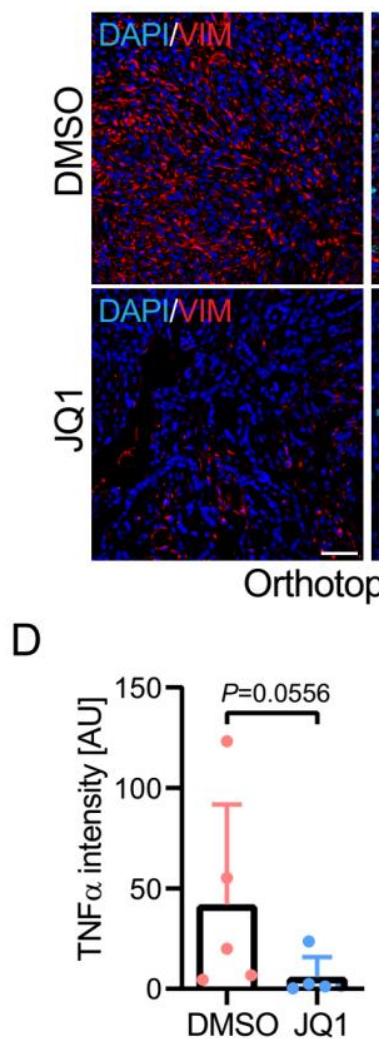

B
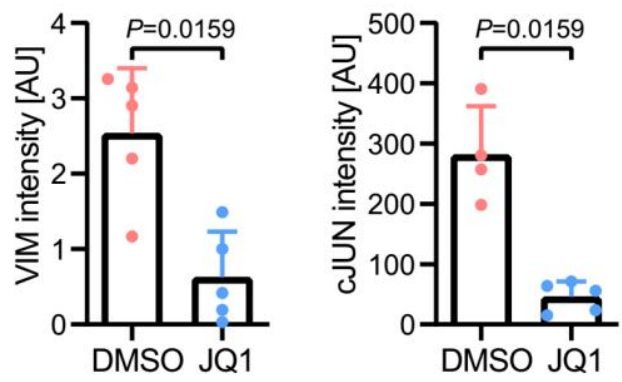

Figure 36. JQ1 alone reduces inflammatory immune microenvironment and EMT factors. (A) Representative images of IF staining for vimentin (VIM), cJUN, TNFa, CCL2, and CD45 in syngeneic KPC tumors with DMSO and JQ1 treatment. (B-E) Intensity quantification of VIM (B), cJUN (C), TNFa (D), and CCL2 (E). Scatter plots show values in arbitrary units (AU) as an average per animal, as well as means \pm s.d. as bar graphs. $n=5$ in DMSO; $n=5$ in JQ1. MannWhitney test was used for statistical analysis. (F) $C D 45^{+}$cells of (A). Scatter plots show average count values per animal per F.o.V. as well as means \pm s.d. as bar graphs. Mann-Whitney test was used for statistical analysis. $n=5$ in DMSO; $n=5$ in JQ1. 


\section{Discussion}

\subsection{The role of TNFa in PDAC plasticity}

Tumor subtype-specific environmental factors play a major role in disease prognosis. In PDAC, the subtype-specific inflammatory immune signals determine distinct prognosis in patients. Recent transcriptional profiling studies in PDAC tumors identified a complex heterogeneity within stromal immune components (activated stroma vs. immune components) and tumor epithelium (CLA vs. BL) (Moffitt et al., 2015; Puleo et al., 2018). CLA subtype is correlated with better prognosis compared to BL subtype (Moffitt et al., 2015). Nevertheless, how subtype-specific molecular events shape the inflammatory immune microenvironment, and hence determines PDAC prognosis remains unclear.

As a key inflammatory cytokine, TNFa was originally identified to induce the necrosis of transplanted methylcholanthrene-induced sarcomas in mice (Carswell et al., 1975). However, subsequent evidence unveiled a paradoxical role of TNFa in tumor promotion (Calcinotto et al., 2012). In ovarian cancer, TNFa was able to promote tumor cell spreading to other organs by upregulating a range of cytokines (Kulbe et al., 2007). Besides, TNFa concentrations was higher in non-small-cell lung cancer patient serum sample; increased TNFa level was associated with high lymphoid and distant metastasis (Shang et al., 2017). Similar findings were reported in PDAC, where TNFa levels were negatively correlated with PDAC patient survival, as revealed by TMA analysis. TNF $\alpha$ neutralization in combination with chemotherapeutics significantly improved mice survival (Zhao et al., 2016).

Based on the notion that TNFa may play a role in promoting PDAC progression, we demonstrated the correlation between high-level TNFa and high-grade/poorly differentiated tumors of KPC mice and PDAC patient biopsies. Mouse orthotopic transplantation model using the CLA and BL cells also confirmed the profound enrichment of TNFa in the BL tumors. Thus, we show a phenotype-dependent expression of TNFa. Addtionally, studies in PDAC suggest a marked correlation of TNFa-related signatures to inflammatory stromal and BL subtypes (Miyabayashi et al., 2020; Puleo et al., 2018; 
Chan-Seng-Yue et al., 2020). For instance, transcriptomic analysis of PDAC patient tumors revealed a significant enrichment of TNFa signaling pathway in the inflammatory stromal component corresponding to BL subtype (Puleo et al., 2018). Additionally, studies from Chan-Seng-Yue et al. and Miyabayashi et al. noted that TNFa-signaling pathway was significantly enriched in the BL tumors and invasive PDAC phenotypes (Miyabayashi et al., 2020; Chan-Seng-Yue et al., 2020). However, whether PDAC subtype-specific TNFa expression restrain or promote disease aggressiveness remains to be established.

We show that exogenous TNFa administration switched W/M differentiated tumors into poorly differentiated BL phenotypic state of a CLA-derived orthotopic mouse model. This phenotypic change was further validated with IF staining for the BL-specific marker KRT81 (Noll et al., 2016; Muckenhuber et al., 2018), which is highly expressed in the TNFa-treated CLA orthotopic tumors, highlighting the significant role of TNFa in tumorpromoting and subtype-switching in PDAC. Besides tumor subtype switch, we also observed that TNFa-induced BL phenotypic state was associated with an increased infiltration of $\mathrm{CD} 45^{+}$immune cells.

TNFa was found to induce the invasive capacity of the CLA cells, concomitant with reduction of a low-grade specific TF KLF5 and induction of EMT inducer ZEB1. ZEB1 is a vital TF known to enhance the invasive and metastatic capacity of PDAC (Krebs et al., 2017). Tumor cells lacking ZEB1 switched from mesenchymal to an epithelial phenotype in PDAC (Wellner et al., 2009). On the other hand, KLF5 was shown to be specifically expressed in the CLA cells as well as low-grade tumors in PDAC (Diaferia et al., 2016). KLF5 plays an essential role in the maintenance of the CLA phenotype via regulating a subset of epithelial gene signatures (Diaferia et al., 2016). There are lineage genes and TFs, which are important for the maintenance of subtype identity (Neph et al., 2012). Studies have uncovered an association of ELF3, HNF1B, and GATA6 with low-grade tumors, and they are characterized as CLA lineage markers (Collisson et al., 2011; Diaferia et al., 2016; Martinelli et al., 2017; Somerville et al., 2018). However, TP63, GLI2 are required for the BL subtype identity (Adams et al., 2019; Somerville et al., 2018). Together, for the first time, we show that TNFa can switch the phenotypic identity by changing the expression of the lineage-specific gene regulatory program. 
Hallmarks of EMT and inflammatory response were significantly enriched in the TNFatreated CLA cells revealed by RNA-seq analysis, in line with our previous observations of TNFa induced BL phenotype in vivo and in vitro. 'KRAS signaling down' and 'myogenesis' pathways induced by TNFa also appeared in the aggressive PDAC phenotypes evidenced by Miyabayashi et al. (Miyabayashi et al., 2020). Additionally, we show that TNFa caused downregulation of majority of the CLA gene signatures including ELF3, FOXQ1, CEACAM6, CEACAM5, as well as AGR2, and upregulation of BL markers such as KRT5, KRT14, and EGFR. Taken together, TNFa was firstly evidenced to induce CLA to inflammatory BL phenotypic switch and favors tumor progression via regulation of epithelial plasticity in PDAC (Figure 37).

CLA subtype

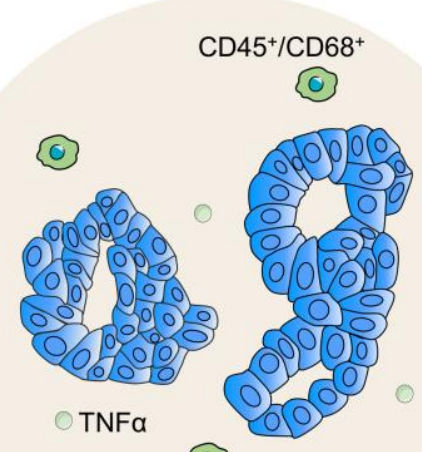

(0)

$\mathrm{TNF}^{\text {low }} /$ Immune low

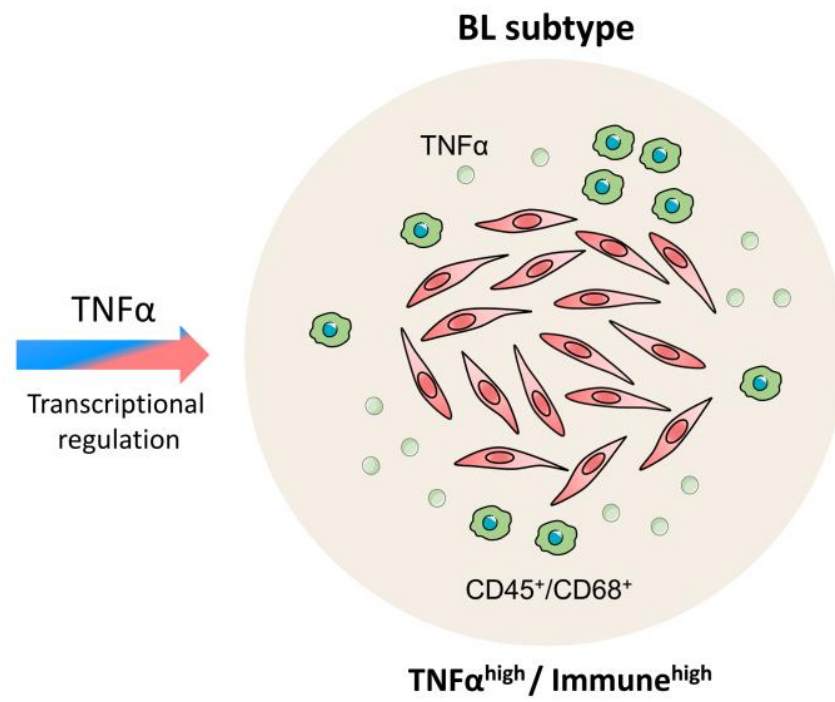

TNF $\alpha^{\text {high }} /$ Immune ${ }^{\text {high }}$

Figure 37. Illustration of TNFa induced CLA to BL subtype switch. CLA PDAC subtype displays well-differentiated tumor phenotype with limited expression of TNF $\alpha$ and $\mathrm{CD} 45^{+}$cells. Conversely, BL subtype has abundant TNFa in tumor tissues. Exogenous TNFa leads to subtype switch from $C L A$ to $B L$ phenotypic state via downregulation of CLA-associated transcriptional network, accompanied by increased infiltration of $C D 45^{+}$cells. 


\subsection{PDAC subtype-dependent stromal microenvironment}

Based on the observations of subtype-dependent expression of TNFa in tumor tissues, the initial hypothesis was that TNFa was produced by BL tumor cells. However surprisingly, contrary to Zho et al. who reported the source of TNFa expression from the PDAC cell lines (i.e. PANC1 and MiaPaCa2 cell lines) (Zhao et al., 2016), we found that none of the PDAC cells including CAPAN1, CAPAN2, PANC1, and MiaPaC2 express TNFa. Though different antibodies were used for flow cytometry analysis, cell lines might differ in the aspects of source and passages. We further validated that these cells do not produce soluble TNFa via ELISA, suggesting that PDAC cells are not the source of TNFa in the BL tumors.

Except for the epithelial cells in the TME, stromal immune counterpart is equally important for modulating subtype identity (Ho et al., 2020). We speculated that TNFa might come from the inflammatory microenvironment of the BL subtype. Examination of human- and murine-specific TNFa revealed that only murine TNFa was detected in the BL orthotopic transplanted mice with no trace of human TNFa, indicating the source of TNFa being host inflammatory microenvironment. In the $\mathrm{BL}$ tumors, $\mathrm{CD} 45^{+}$and $\mathrm{CD}^{2} 8^{+}$cells were highly infiltrated relative to the CLA tumors. Recently, single-cell analysis in PDAC mouse models unveiled that macrophage was the most predominant cell population in the late stage of the disease (Hosein et al., 2019). Thus, it is highly likely that TNFa-producing $\mathrm{CD}^{+} 8^{+}$macrophages present in PDAC in a subtype-dependent manner.

In the TME, CCL2 is a chemotactic cytokine known to recruits macrophages through CCL2-CCR2 signaling (Nagarsheth et al., 2017). Mounting evidence have reported that CCL2 signaling-mediated macrophages contribute to tumor progression and metastasis in multiple cancers (Chen et al., 2018; Fader et al., 2010; Qian et al., 2011; Zhang et al., 2010). To take an example of breast cancer, CCL2 was reported to recruit macrophages to facilitate tumor cell metastasize to the lung (Qian et al., 2011). However, after blockage of CCL2 by anti-CCL2 antibody, infiltrated macrophages were dramatically reduced, as well as lung metastases. Consequently, mice survival was significantly prolonged (Qian et al., 2011). Moreover, a high level of CCL2 was associated with poor prognosis in lung 
cancer, ovarian cancer, as well as breast cancer (Li et al., 2018; Ueno et al., 2000). In our study, levels of several cytokines including CCL2 were extremely high in the BL cells analyzed by RNA-seq data obtained from publicly available dataset (Bailey et al., 2016). High-level CCL2 in the BL cells was further validated by human cytokine profiler kit. Furthermore, a strong association of CCL2 with the squamous (i.e. BL) PDAC subtype (Puleo et al., 2018) could be seen. Thus, we show that different PDAC subtype links to distinct TME. Compared to CLA, BL subtype has an inflammatory TME with high expression of CCL2 and infiltration of TNFa-producing $\mathrm{CD}^{2} 8^{+}$macrophages.

Similarly, the work from $\mathrm{Li}$ et al. highlighted that tumor intrinsic cytokine determines the immune heterogeneity of PDAC ( $\mathrm{Li}$ et al., 2018). They identified CXCL1 as a pivotal intrinsic factor that changes the subtype identity from $T$ cell-low to $T$ cell-high tumors. Besides, inhibition of colony stimulating factor 1 receptor (CSF1R) was able to alter the composition of TME (Candido et al., 2018). Ligand CSF1 mediated signaling is important for the differentiation and maintenance of macrophage (Sehgal et al., 2018). Targeting of CSF1R led to significantly reduction of macrophages in tumor tissues. Therefore, tumor switched from BL to CLA phenotype and mice survived longer (Candido et al., 2018), suggesting the significant role of macrophage in subtype identity.

\subsection{The role of JUN/AP1 family in tumor plasticity}

AP1 transcription factor has been extensively studied of its function regarding the regulation of inflammatory response (Lopez-Bergami et al., 2010). However, the implication of AP1 family TF complexes in PDAC aggressiveness remains elusive. AP1 TF complexes consist of JUN (cJUN, JUNB, JUND), Fos (c-FOs, FOSB, FRA1, FRA2), activating transcription factor (ATF) (ATF2, ATF3, B-ATF, JDP1, JDP2), as well as musculoaponeurotic fibrosarcoma (MAF) (c-Maf, MafB, MafA, MafG/F/K and Nrl) (Shaulian and Karin, 2002). They form either homo- or heterodimeric protein complexes (e.g. cJUN-cJUN, cJUN-FOS) for enhancing DNA binding to regulate their gene targets (Eckert et al., 2013). In recent years, emerging studies unveiled the potential role of AP1 in regulating tumor phenotypic identity (Diaferia et al., 2016; Milan et al., 2019; Roe et al., 2017). 
We identified that JUN/AP1 pathway was significantly associated with the PDAC subtype identity. cJUN/AP1 and JUNB/AP1 were mutually exclusively expressed in the PDAC subtype specific manner. Our in vitro and in vivo findings suggest that cJUN is mainly involved in the maintenance of BL-inflammatory program, whereas JUNB is exclusively expressed in the CLA tumors. In line with our findings, Diaferia et al. have identified high expression of JUNB in low-grade tumors based on immunochemistry staining (Diaferia et al., 2016), confirming the correlation of JUNB with the CLA subtype. Although, a recent study has shown that cJUN/AP1 induces inflammatory program in chronic pancreatitis (Cobo et al., 2018), the transcriptional activation of cJUN in PDAC subtype specificity remains unresolved.

By intersecting JUNB or cJUN targets with CLA-related or BL-related ATAC-peaks respectively, we find that 'cell-cell adhesion regulation' and 'epithelial cell differentiation' gene sets were enriched in JUNB/CLA-related regions, which support our findings that JUNB restricted to well-differentiated tumors. On the other hand, pathways of 'response to growth factor', 'leukocyte migration', as well as 'regulation of cytokine production' were enriched in cJUN/BL-related regions, suggesting the role of cJUN in immune regulation. Consistently, we detected strong occupancy of JUNB on $\mathrm{CDH} 1$ at promoter and enhancer sites, associated with H3K27ac occupancy. CDH1 encodes for ECAD, which is a transmembrane protein, involved in the formation of adherens junctions to mediate cell to cell connections (van Roy and Berx, 2008). ECAD is typically expressed in the epithelial cells, playing an essential role in the maintenance of epithelial structure (Natalwala et al., 2008). In the BL cells, we show that cJUN occupies both promoter and enhancer loci of VIM. VIM is a family member of intermediate filaments, maintaining mesenchymal features (Ivaska et al., 2007). These findings support the idea that JUNB might involve in maintenance of epithelial differentiation in the CLA subtype, while cJUN is, in fact, required for the tumor dedifferentiation in the BL subtype.

Together, we show that cJUN and JUNB exert distinct functions in PDAC subtype maintenance. We proposed a model to elaborate cJUN- and JUNB- mediated PDAC subtype identity (Figure 38). 

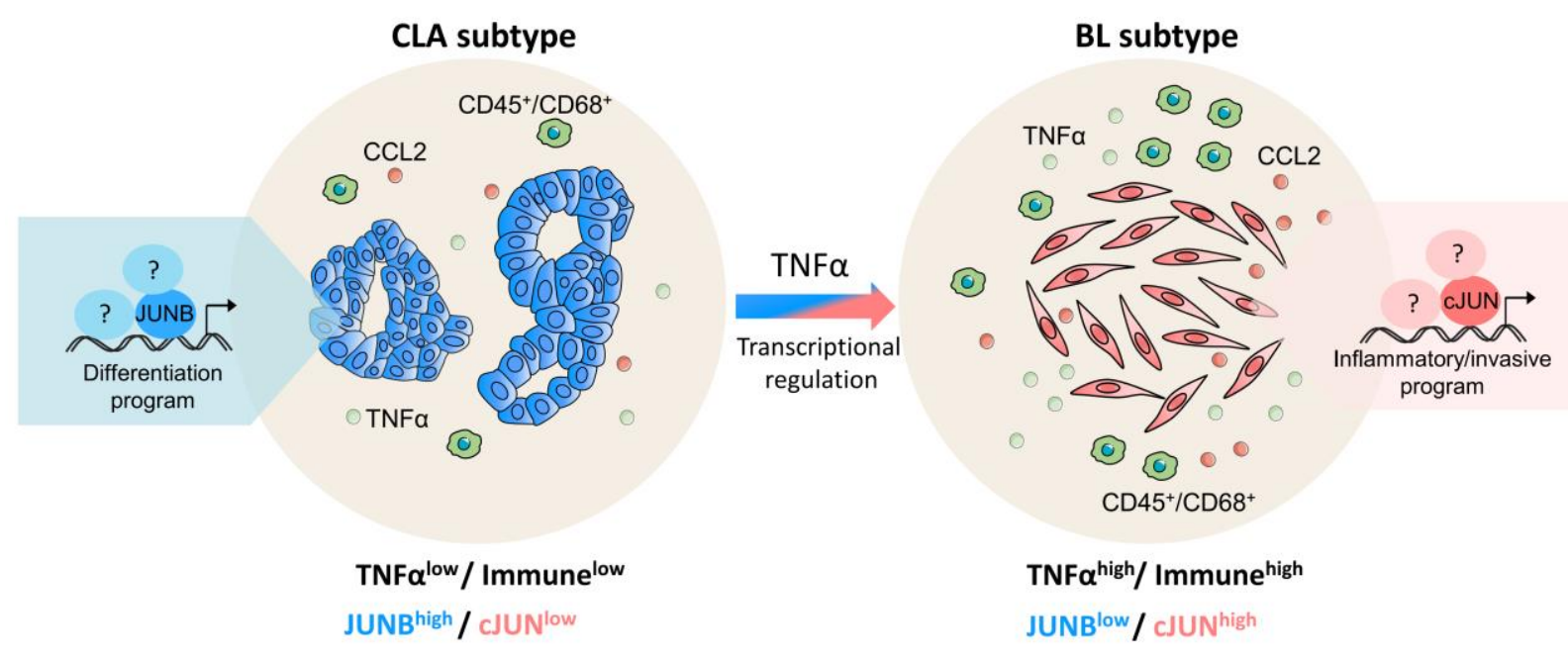

Figure 38. JUNB and cJUN in controlling PDAC subtype identity. JUNB is selectively expressed in the CLA PDAC subtype and maintain the epithelial differentiation. cJUN is restricted to the BL PDAC subtype which is highly infiltrated with $\mathrm{CD} 68^{+}$cells. The possibility of other lineage transcription factors cooperative with JUNB or cJUN still needs to be tested.

\section{4 cJUN-regulatory network in macrophages recruitment and therapy resistance}

We show that cJUN-OE cells displayed an invasive phenotype in CLA PDAC cells. Our findings align with the previous report where cJUN overexpression showed similar phenotype in MCF-7 breast cancer cells (Smith et al., 1999). Notably, MCF7 cell line identified as luminal A subtype (Adelaide et al., 2007), which displays identical phenotype to the PDAC low-grade/CLA subtype (Adelaide et al., 2007). Consistently, another finding in breast cancer model showed that loss of cJUN activity associated with reduced tumor cell migration and invasive behavior (Jiao et al., 2010). Thus, cJUN promotes the shift of CLA tumors into more invasive BL phenotypic state. Moreover, $B L$ tumor aggressiveness is often associated with increased chemoresistance profile in PDAC (Aung et al., 2018). Since cJUN-OE switched cells towards a more BL phenotype, we proposed that CJUNOE cells might exhibit chemoresistant properties. Of note, PDAC patients with CLA 
tumors respond efficiently to chemotherapy, in particular with m-FOLFIRINOX and nappaclitaxel/gemcitabine (Aung et al., 2018). We compared IC S $_{50}$ of oxaliplatin, SN38 (an activate metabolite of irinotecan) that are the active components of FLOFIRINOX, as well as gemcitabine in cJUN-OE cells. Oxaliplatin was designed based on platinum, forming DNA-platinum adducts to inhibit DNA replication and induce cell apoptosis (Zoetemelk et al., 2020). While irinotecan is a topoisomerase inhibitor and it slightly inhibits DNA synthesis (Zoetemelk et al., 2020). However, the effect was much more augmented when it is transformed into an activated metabolite SN38. SN38 accelerates DNA damage via generating chromosome aberrations and ultimately leads to apoptosis (Zoetemelk et al., 2020). cJUN-OE cells showed more resistance to these drugs compared to EV cells. Similarly, Lipner et al. reported that cJUN is essential for conferring resistance to FOLFOX (combination of 5-FU and oxaliplatin) treatment in PDAC mouse models (Lipner et al., 2020). Furthermore, lentiviral-mediated cJUN depletion significantly increased the sensitivity of PDAC cells to FOLFOX (Lipner et al., 2020), confirming the crucial role of cJUN in chemoresistance, thereby maintaining BL phenotypic state.

Besides cJUN functions in the neoplastic-epithelial plasticity, we also identified its crucial role in the recruitment of inflammatory immune cells. We hypothesized that cJUN might transcriptionally regulate CCL2 for the recruitment of TNFa-producing macrophages in the BL tumors. Our novel findings show that cJUN directly controls CCL2 expression via enhancer-promoter regulation in BL PDAC cells. Importantly, cJUN-mediated CCL2 expressions is found in the established PDAC cells as well as cJUN-high cell lines isolated from patient-derived xenograft (PDX). Together, these findings suggest that cJUN positively regulates CCL2 for the maintenance of TNFa-mediated inflammatory program in the BL PDAC subtype. Of note, we showed that high expressions of TNFa is linked to inflammatory and poorly differentiated/BL phenotypic state.

Interestingly, our results show that TNFa induces cJUN-CCL2 feed-forward loop in CLA PDAC subtype. Constitutive expression of TNFa in the CLA cells led to a highly invasive/BL phenotypic identity, which is functionally similar to the cJUN-OE CLA PDAC cell lines. Importantly, loss of cJUN significantly suppressed the TNFa induced CCL2, indicating that cJUN is essential for the TNFa mediated upregulation of CCL2.Together, 
we identified a cJUN-driven inflammatory BL program, which is essential for maintenance of TNFa-mediated PDAC aggressiveness (Figure 39).
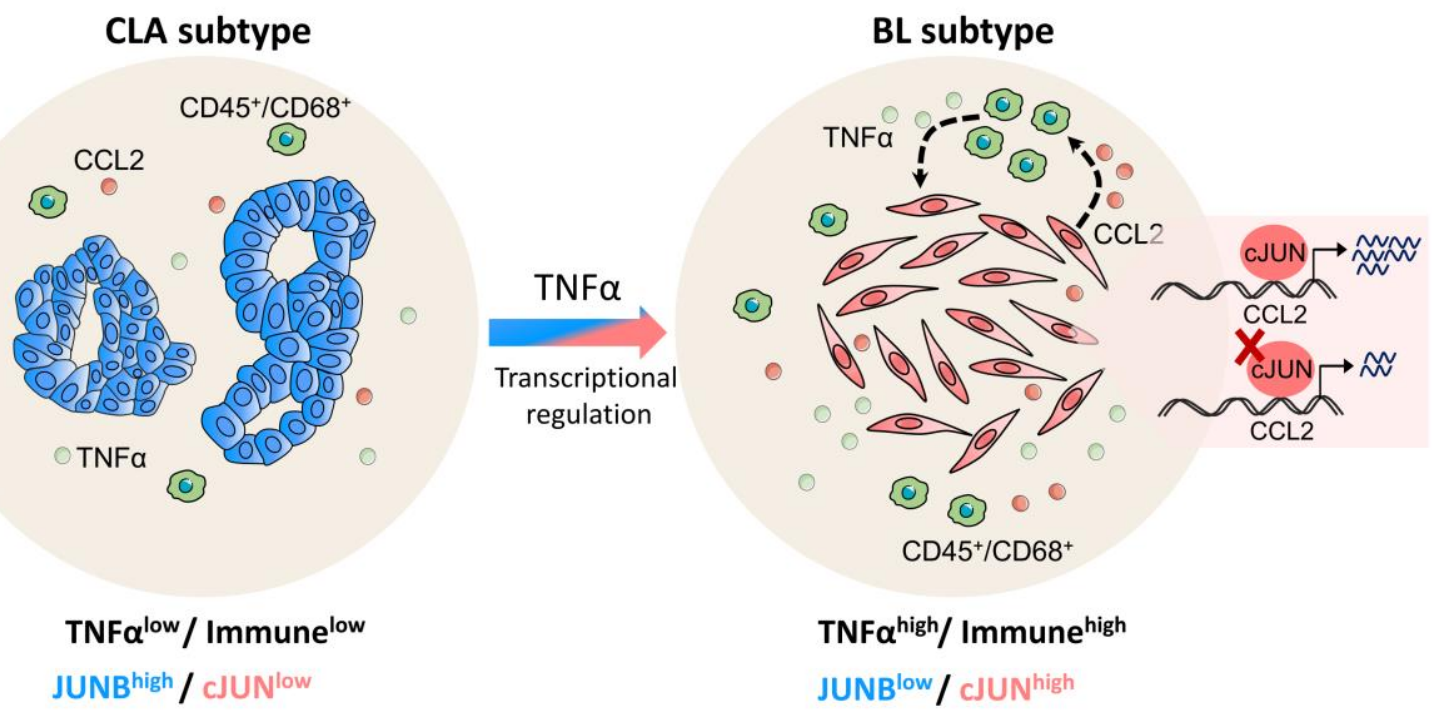

Figure 39. Illustration model of CJUN-CCL2-TNFa positive feedforward loop. cJUN activates CCL2 to recruit $\mathrm{CD} 8^{+}$macrophages which secrete TNFa, and in turn, fostering tumor cells and activating CJUN-CCL2 signaling axis to promote a worse phenotype.

\subsection{Monotherapy of anti-TNFa is not ideal for PDAC}

Anti-TNFa monotherapy has been approved in clinical use for many types of chronic inflammatory disease including rheumatoid arthritis, Crohn's disease, psoriatic arthritis, ankylosing spondylitis, ulcerative colitis, as well as severe psoriasis (Feldmann, 2002; Jackson, 2007; Thalayasingam and Isaacs, 2011). To date, there are five distinct monoclonal antibodies available for targeting TNFa: etanercept, infliximab, adalimumab, golimumab, and certolizumab (Thalayasingam and Isaacs, 2011). Despite the promising effect of anti-TNF $\alpha$ conferred in the inflammatory disease, whether it is effective for cancer treatment is still debatable. 
In our study, anti-TNFa treatment did not improve the overall survival of syngeneic KPC mice, which is a highly aggressive immunocompetent mouse model. We anticipated that TNFa monotherapy might switch the BL tumors into more favorable CLA-like phenotype. However, neither tumor differentiation nor recruitment of inflammatory immune cells $\left(\mathrm{CD} 45^{+}\right.$and $\left.\mathrm{CD}^{2} 8^{+}\right)$were altered following TNFa monotherapy. There could be two major reasons for the failure of anti-TNFa monotherapy: (i) tumors developed from syngeneic KPC mice are highly heterogeneous and surrounded by dense stroma tissues including fibroblasts, extracellular matrix, and vasculature (Neesse et al., 2011; Ying et al., 2016). Desmoplastic reaction makes it difficult for TNFa neutralizing antibodies to penetrate and reach PDAC tumors. Similarly, CCL2 neutralizing antibody alone did not improve the survival of PDAC mouse model (Kalbasi et al., 2017); (ii) KPC-derived tumors display extremely aggressive phenotype, which constitutively expresses cJUN-mediated CCL2TNFa axis. Therefore, targeting TNFa alone is not sufficient to prevent the overall tumor progression. Therefore, targeting cJUN alone or in combination with TNFa monotherapy may improve the outcome of BL tumors.

\subsection{Preclinical inhibition of BRD4 confers favorable prognosis of BL tumors}

Considering that anti-TNFa therapy failed to improve the prognosis in PDAC, and there was no pharmacological inhibitor available to specifically target cJUN, we assumed that a potential chromatin regulator could be serve as a candidate for the blocking cJUNmediated signaling axis. Interestingly, our data showed that chromatin regulator BRD4 was highly enriched in the BL subtype (Diaferia et al., 2016). BRD4 belongs to bromodomain and extraterminal domain (BET) family, it is a chromatin remodeler that functions as a chromatin 'reader' to recognizes the acetylated histones and regulate gene expression (Belkina and Denis, 2012; Wu et al., 2013). Study has shown that BRD4 was highly expressed in the late stage of PDAC mouse tumors (Hosein et al., 2019). Most recently, the work from Shu et al. revealed that in breast cancer patients, BRD4 was significantly higher expressed in the basal (likely to BL PDAC) tumors compared to luminal (likely to CLA PDAC) tumors (Shu et al., 2020). 
This enrichment analysis gave us an indication that there might be a regulation between BRD4 and cJUN. And if so, BRD4 could be exploit as a therapeutic target for inhibiting cJUN. JQ1 is a small molecular inhibitor, which competitively binds to the pocket of the bromodomain and replaces the BRD4 protein from acetylated histones (Filippakopoulos et al., 2010). By reanalyzing public ChIP-seq data in acute myeloid leukemia (AML) cells (Bhagwat et al., 2016), we showed that JQ1 led to decreased BRD4 occupancy at enhancer regions of cJUN. Consistently, JQ1 resulted in reduced occupancy of BRD4 at cJUN enhancer sites in PDAC, as validated by ChIP-qPCR. The positive regulation of BRD4 on cJUN was confirmed by reduced expression of cJUN upon abrogation of BRD4 by siRNA. As expected, BET inhibitor JQ1 led to reduction of cJUN in the BL cells.

Mazur et al. showed that JQ1 resulted in reduced expression of $\mathrm{CD} 45^{+}$cells as well as $\mathrm{F} 4 / 80^{+}$macrophages. The JQ1 therapy alone, or in combination with histone deacetylase (HDAC) inhibitor, significantly improved the survival of PDAC mouse model (Mazur et al., 2015, Andricovich et al., 2018). Thus, pharmacological inhibition of BRD4 via JQ1 significantly improve the pre-clinical outcome of PDAC. Importantly, we show that JQ1 monotherapy switched the highly aggressive/BL tumors into more CLA-like phenotypic state, which was not feasible with anti-TNFa monotherapy. We provide a very strong evidence that pharmacological inhibition of BRD4 via JQ1 significantly disrupted cJUNmediated BL inflammatory program. Together, our findings suggest that JQ1 therapy could specifically be applied for cJUN ${ }^{\text {high }} / T N F a^{\text {high }}$ BL PDAC tumors (Figure 40). 

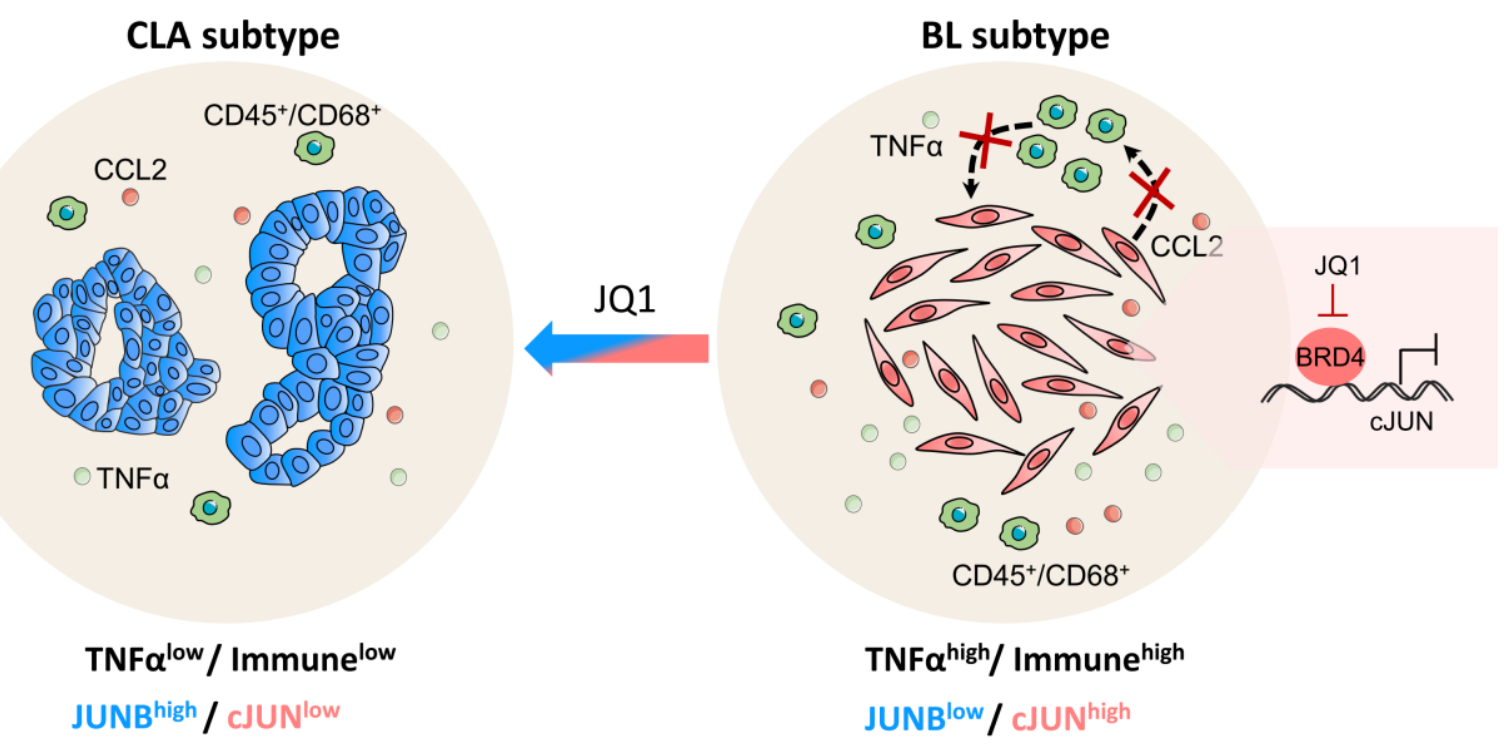

Figure 40. Schematic illustration of JQ1 mediated favorable prognosis in PDAC. cJUN could be targeted by inhibiting of BRD4 with BET inhibitor JQ1, thus, leading to well differentiated phenotype and less infiltrates for favoring a better prognosis.

\subsection{Concluding remarks}

Complex tumor stroma heterogeneity and cancer cell plasticity are the major challenges in PDAC poor prognosis and therapy resistance. Recent molecular subtyping of PDAC has significantly improved the molecular understanding of this lethal disease. Studies have shown that lineage-specific TFs and their associated gene signatures tightly control PDAC subtype identity and disease aggressiveness. In addition, the tumor subtype identity can be shaped by external stimuli from the existing tumor immune microenvironment. However, the precise mechanism of how this regulatory program operates is still largely unknown.

We provide a set of compelling evidence that explains how neoplastic-specific transcriptional reprograming modulates intra-tumor immune heterogeneity, which profoundly influences prognostic and therapeutic outcomes. Pharmacological inhibition 
can indeed modulate these epigenetic-driven transcriptional networking and allow us to induce a favorable subtype switch.

Collectively, we report that:

D High expression of TNFa is restricted to the high-grade/poorly differentiated $\mathrm{BL}$ subtype, whereas CLA subtype is low-grade/well-to-moderately differentiated with low-proinflammatory immune infiltrations;

$>$ Two antithetical and mutually exclusive AP1-driven transcriptional programs determine subtype identity and, thus, prognosis. JUNB/AP1 controls CLA tumor identity, whereas cJUN/AP1 controls the BL phenotype;

> BL-restricted cJUN maintains a pro-inflammatory gene expression program by controlling chromatin accessibility and enhancer potentiation; it thus recruits $\mathrm{TNFa}^{+}$macrophages in the microenvironment via CCL2 to sustain an inflammatory niche;

> CLA-restricted JUNB cannot support this pro-inflammatory identity and precludes macrophages recruitment; however, TNFa treatment suffices for switching CLAtumors towards the aggressive BL identity;

BRD4-bound enhancers support the cJUN-CCL2-TNFa axis, and hence, macrophages recruitment. Treatment with a known BET inhibitor disrupts this axis and allows reprogramming of poorly differentiated/BL-tumors into more differentiated/CLA ones that are amenable to chemotherapy. 


\section{References}

Adams, C.R., Htwe, H.H., Marsh, T., Wang, A.L., Montoya, M.L., Subbaraj, L., Tward, A.D., Bardeesy, N., and Perera, R.M. (2019). Transcriptional control of subtype switching ensures adaptation and growth of pancreatic cancer. eLife 8 .

Adamska, A., Domenichini, A., and Falasca, M. (2017). Pancreatic Ductal Adenocarcinoma: Current and Evolving Therapies. International journal of molecular sciences 18.

Adelaide, J., Finetti, P., Bekhouche, I., Repellini, L., Geneix, J., Sircoulomb, F., CharafeJauffret, E., Cervera, N., Desplans, J., Parzy, D., et al. (2007). Integrated profiling of basal and luminal breast cancers. Cancer research 67, 11565-11575.

Aggarwal, B.B., Moffat, B., and Harkins, R.N. (1984). Human lymphotoxin. Production by a lymphoblastoid cell line, purification, and initial characterization. The Journal of biological chemistry 259, 686-691.

Andre, F., and Pusztai, L. (2006). Molecular classification of breast cancer: implications for selection of adjuvant chemotherapy. Nature clinical practice. Oncology 3, 621-632.

Anders, S., Pyl, P.T., and Huber, W. (2015). HTSeq--a Python framework to work with high-throughput sequencing data. Bioinformatics 31,166-169.

Andricovich, J., Perkail, S., Kai, Y., Casasanta, N., Peng, W., and Tzatsos, A. (2018). Loss of KDM6A Activates Super-Enhancers to Induce Gender-Specific Squamous-like Pancreatic Cancer and Confers Sensitivity to BET Inhibitors. Cancer cell 33, 512-526 e518.

Aung, K.L., Fischer, S.E., Denroche, R.E., Jang, G.H., Dodd, A., Creighton, S., Southwood, B., Liang, S.B., Chadwick, D., Zhang, A., et al. (2018). Genomics-Driven Precision Medicine for Advanced Pancreatic Cancer: Early Results from the COMPASS Trial. Clinical cancer research : an official journal of the American Association for Cancer Research 24, 1344-1354.

Bailey, P., Chang, D.K., Nones, K., Johns, A.L., Patch, A.M., Gingras, M.C., Miller, D.K., Christ, A.N., Bruxner, T.J., Quinn, M.C., et al. (2016). Genomic analyses identify molecular subtypes of pancreatic cancer. Nature 531, 47-52.

Balkwill, F. (2006). TNF-alpha in promotion and progression of cancer. Cancer metastasis reviews 25, 409-416.

Balkwill, F. (2009). Tumour necrosis factor and cancer. Nature reviews. Cancer 9, 361371. 
Bartsch, D. K., Sina-Frey, M., Lang, S., Wild, A., Gerdes, B., Barth, P., Kress, R., Grutzmann, R., Colombo-Benkmann, M., Ziegler, A., et al. (2002). CDKN2A germline mutations in familial pancreatic cancer. Annals of surgery $236,730-737$.

Belkina, A.C., and Denis, G.V. (2012). BET domain co-regulators in obesity, inflammation and cancer. Nature reviews. Cancer 12, 465-477.

Bhagwat, A.S., Roe, J.S., Mok, B.Y.L., Hohmann, A.F., Shi, J., and Vakoc, C.R. (2016). BET Bromodomain Inhibition Releases the Mediator Complex from Select cis-Regulatory Elements. Cell reports 15, 519-530.

Biffi, G., Oni, T.E., Spielman, B., Hao, Y., Elyada, E., Park, Y., Preall, J., and Tuveson, D.A. (2019). IL1-Induced JAK/STAT Signaling Is Antagonized by TGFbeta to Shape CAF Heterogeneity in Pancreatic Ductal Adenocarcinoma. Cancer discovery 9, 282-301.

Brahmer, J., Reckamp, K.L., Baas, P., Crino, L., Eberhardt, W.E., Poddubskaya, E., Antonia, S., Pluzanski, A., Vokes, E.E., Holgado, E., et al. (2015). Nivolumab versus Docetaxel in Advanced Squamous-Cell Non-Small-Cell Lung Cancer. The New England journal of medicine $373,123-135$.

Brunton, H., Caligiuri, G., Cunningham, R., Upstill-Goddard, R., Bailey, U.M., Garner, I.M., Nourse, C., Dreyer, S., Jones, M., Moran-Jones, K., et al. (2020). HNF4A and GATA6 Loss Reveals Therapeutically Actionable Subtypes in Pancreatic Cancer. Cell reports 31, 107625.

Burris, H. A., 3rd, Moore, M. J., Andersen, J., Green, M. R., Rothenberg, M. L., Modiano, M. R., Cripps, M. C., Portenoy, R. K., Storniolo, A. M., Tarassoff, P., et al. (1997). Improvements in survival and clinical benefit with gemcitabine as first-line therapy for patients with advanced pancreas cancer: a randomized trial. Journal of clinical oncology : official journal of the American Society of Clinical Oncology 15, 2403-2413.

Cancer Genome Atlas Research Network. Electronic address, a.a.d.h.e., and Cancer Genome Atlas Research, N. (2017). Integrated Genomic Characterization of Pancreatic Ductal Adenocarcinoma. Cancer cell 32, 185-203 e113.

Calcinotto, A., Grioni, M., Jachetti, E., Curnis, F., Mondino, A., Parmiani, G., Corti, A., and Bellone, M. (2012). Targeting TNF-alpha to neoangiogenic vessels enhances lymphocyte infiltration in tumors and increases the therapeutic potential of immunotherapy. Journal of immunology 188, 2687-2694.

Candido, J.B., Morton, J.P., Bailey, P., Campbell, A.D., Karim, S.A., Jamieson, T., Lapienyte, L., Gopinathan, A., Clark, W., McGhee, E.J., et al. (2018). CSF1R(+) Macrophages Sustain Pancreatic Tumor Growth through $T$ Cell Suppression and Maintenance of Key Gene Programs that Define the Squamous Subtype. Cell reports 23, 1448-1460. 
Carswell, E.A., Old, L.J., Kassel, R.L., Green, S., Fiore, N., and Williamson, B. (1975). An endotoxin-induced serum factor that causes necrosis of tumors. Proceedings of the National Academy of Sciences of the United States of America 72, 3666-3670.

Chan-Seng-Yue, M., Kim, J.C., Wilson, G.W., Ng, K., Figueroa, E.F., O'Kane, G.M., Connor, A.A., Denroche, R.E., Grant, R.C., McLeod, J., et al. (2020). Transcription phenotypes of pancreatic cancer are driven by genomic events during tumor evolution. Nature genetics 52, 231-240.

Chen, C., He, W., Huang, J., Wang, B., Li, H., Cai, Q., Su, F., Bi, J., Liu, H., Zhang, B., et al. (2018). LNMAT1 promotes lymphatic metastasis of bladder cancer via CCL2 dependent macrophage recruitment. Nature communications 9, 3826.

Chen, E.Y., Tan, C.M., Kou, Y., Duan, Q., Wang, Z., Meirelles, G.V., Clark, N.R., and Ma'ayan, A. (2013). Enrichr: interactive and collaborative HTML5 gene list enrichment analysis tool. BMC bioinformatics 14, 128.

Cobo, I., Martinelli, P., Flandez, M., Bakiri, L., Zhang, M., Carrillo-de-Santa-Pau, E., Jia, J., Sanchez-Arevalo Lobo, V.J., Megias, D., Felipe, I., et al. (2018). Transcriptional regulation by NR5A2 links differentiation and inflammation in the pancreas. Nature 554, 533-537.

Colleoni, M., Rotmensz, N., Maisonneuve, P., Mastropasqua, M.G., Luini, A., Veronesi, P., Intra, M., Montagna, E., Cancello, G., Cardillo, A., et al. (2012). Outcome of special types of luminal breast cancer. Annals of oncology : official journal of the European Society for Medical Oncology 23, 1428-1436.

Collisson, E.A., Sadanandam, A., Olson, P., Gibb, W.J., Truitt, M., Gu, S., Cooc, J., Weinkle, J., Kim, G.E., Jakkula, L., et al. (2011). Subtypes of pancreatic ductal adenocarcinoma and their differing responses to therapy. Nature medicine 17, 500-503.

Conroy, T., Desseigne, F., Ychou, M., Bouche, O., Guimbaud, R., Becouarn, Y., Adenis, A., Raoul, J. L., Gourgou-Bourgade, S., de la Fouchardiere, C., et al. (2011). FOLFIRINOX versus gemcitabine for metastatic pancreatic cancer. The New England journal of medicine $364,1817-1825$.

Diaferia, G.R., Balestrieri, C., Prosperini, E., Nicoli, P., Spaggiari, P., Zerbi, A., and Natoli, G. (2016). Dissection of transcriptional and cis-regulatory control of differentiation in human pancreatic cancer. The EMBO journal 35, 595-617.

Dobin, A., Davis, C.A., Schlesinger, F., Drenkow, J., Zaleski, C., Jha, S., Batut, P., Chaisson, M., and Gingeras, T.R. (2013). STAR: ultrafast universal RNA-seq aligner. Bioinformatics 29, 15-21. 
Eckert, R.L., Adhikary, G., Young, C.A., Jans, R., Crish, J.F., Xu, W., and Rorke, E.A. (2013). AP1 transcription factors in epidermal differentiation and skin cancer. Journal of skin cancer 2013, 537028.

Egberts, J.H., Cloosters, V., Noack, A., Schniewind, B., Thon, L., Klose, S., Kettler, B., von Forstner, C., Kneitz, C., Tepel, J., et al. (2008). Anti-tumor necrosis factor therapy inhibits pancreatic tumor growth and metastasis. Cancer research 68, 1443-1450.

Ellenrieder, V., Konig, A., and Seufferlein, T. (2016). Current Standard and Future Perspectives in First- and Second-Line Treatment of Metastatic Pancreatic Adenocarcinoma. Digestion 94, 44-49.

Ellis, C.A., and Clark, G. (2000). The importance of being K-Ras. Cellular signalling 12, 425-434.

Fader, A.N., Rasool, N., Vaziri, S.A., Kozuki, T., Faber, P.W., Elson, P., Biscotti, C.V., Michener, C.M., Rose, P.G., Rojas-Espaillat, L., et al. (2010). CCL2 expression in primary ovarian carcinoma is correlated with chemotherapy response and survival outcomes. Anticancer research 30, 4791-4798.

Feldmann, M. (2002). Development of anti-TNF therapy for rheumatoid arthritis. Nature reviews. Immunology 2, 364-371.

Filippakopoulos, P., Qi, J., Picaud, S., Shen, Y., Smith, W.B., Fedorov, O., Morse, E.M., Keates, T., Hickman, T.T., Felletar, I., et al. (2010). Selective inhibition of BET bromodomains. Nature 468, 1067-1073.

Finer, M.H., Dull, T.J., Qin, L., Farson, D., and Roberts, M.R. (1994). kat: a high-efficiency retroviral transduction system for primary human T lymphocytes. Blood 83, 43-50.

Ford, E., Nikopoulou, C., Kokkalis, A., and Thanos, D. (2014). A method for generating highly multiplexed ChIP-seq libraries. BMC research notes 7, 312.

Garon, E.B., Rizvi, N.A., Hui, R., Leighl, N., Balmanoukian, A.S., Eder, J.P., Patnaik, A., Aggarwal, C., Gubens, M., Horn, L., et al. (2015). Pembrolizumab for the treatment of non-small-cell lung cancer. The New England journal of medicine 372, 2018-2028.

Ghaneh, P., Costello, E., and Neoptolemos, J.P. (2008). Biology and management of pancreatic cancer. Postgraduate medical journal 84, 478-497.

Golan, T., Hammel, P., Reni, M., Van Cutsem, E., Macarulla, T., Hall, M.J., Park, J.O., Hochhauser, D., Arnold, D., Oh, D.Y., et al. (2019). Maintenance Olaparib for Germline BRCA-Mutated Metastatic Pancreatic Cancer. The New England journal of medicine 381, 317-327. 
Goldstein, D., El-Maraghi, R.H., Hammel, P., Heinemann, V., Kunzmann, V., Sastre, J., Scheithauer, W., Siena, S., Tabernero, J., Teixeira, L., et al. (2015). nab-Paclitaxel plus gemcitabine for metastatic pancreatic cancer: long-term survival from a phase III trial. Journal of the National Cancer Institute 107.

Grivennikov, S.I., Greten, F.R., and Karin, M. (2010). Immunity, inflammation, and cancer. Cell 140, 883-899.

Guerra, C., Schuhmacher, A.J., Canamero, M., Grippo, P.J., Verdaguer, L., PerezGallego, L., Dubus, P., Sandgren, E.P., and Barbacid, M. (2007). Chronic pancreatitis is essential for induction of pancreatic ductal adenocarcinoma by K-Ras oncogenes in adult mice. Cancer cell 11, 291-302.

Halbrook, C.J., Pontious, C., Kovalenko, I., Lapienyte, L., Dreyer, S., Lee, H.J., Thurston, G., Zhang, Y., Lazarus, J., Sajjakulnukit, P., et al. (2019). Macrophage-Released Pyrimidines Inhibit Gemcitabine Therapy in Pancreatic Cancer. Cell metabolism 29, 13901399 e1396.

Hale, M.A., Swift, G.H., Hoang, C.Q., Deering, T.G., Masui, T., Lee, Y.K., Xue, J., and MacDonald, R.J. (2014). The nuclear hormone receptor family member NR5A2 controls aspects of multipotent progenitor cell formation and acinar differentiation during pancreatic organogenesis. Development 141, 3123-3133.

Hartwig, W., Werner, J., Jager, D., Debus, J., and Buchler, M.W. (2013). Improvement of surgical results for pancreatic cancer. The Lancet. Oncology 14, e476-e485.

Hessmann, E., Buchholz, S.M., Demir, I.E., Singh, S.K., Gress, T.M., Ellenrieder, V., and Neesse, A. (2020). Microenvironmental Determinants of Pancreatic Cancer. Physiological reviews 100, 1707-1751.

Hezel, A. F., Kimmelman, A. C., Stanger, B. Z., Bardeesy, N., \& DePinho, R. A. (2006). Genetics and biology of pancreatic ductal adenocarcinoma. Genes and Development, 20(10), 1218-1249.

Hingorani, S. R., Petricoin, E. F., Maitra, A., Rajapakse, V., King, C., Jacobetz, M. A., Ross, S., Conrads, T. P., Veenstra, T. D., Hitt, B. A., et al. (2003). Preinvasive and invasive ductal pancreatic cancer and its early detection in the mouse. Cancer cell 4 , 437-450.

Hingorani, S.R., Wang, L., Multani, A.S., Combs, C., Deramaudt, T.B., Hruban, R.H., Rustgi, A.K., Chang, S., and Tuveson, D.A. (2005). Trp53R172H and KrasG12D cooperate to promote chromosomal instability and widely metastatic pancreatic ductal adenocarcinoma in mice. Cancer cell 7, 469-483. 
Ho, W.J., Jaffee, E.M., and Zheng, L. (2020). The tumour microenvironment in pancreatic cancer - clinical challenges and opportunities. Nature reviews. Clinical oncology 17, 527540.

Hodi, F.S., O'Day, S.J., McDermott, D.F., Weber, R.W., Sosman, J.A., Haanen, J.B., Gonzalez, R., Robert, C., Schadendorf, D., Hassel, J.C., et al. (2010). Improved survival with ipilimumab in patients with metastatic melanoma. The New England journal of medicine 363, 711-723.

Hosein, A.N., Huang, H., Wang, Z., Parmar, K., Du, W., Huang, J., Maitra, A., Olson, E., Verma, U., and Brekken, R.A. (2019). Cellular heterogeneity during mouse pancreatic ductal adenocarcinoma progression at single-cell resolution. $\mathrm{JCl}$ insight 5.

Hruban, R.H., Adsay, N.V., Albores-Saavedra, J., Compton, C., Garrett, E.S., Goodman, S.N., Kern, S.E., Klimstra, D.S., Kloppel, G., Longnecker, D.S., et al. (2001). Pancreatic intraepithelial neoplasia: a new nomenclature and classification system for pancreatic duct lesions. The American journal of surgical pathology 25, 579-586.

Huang, H., Zhang, Y., Gallegos, V., Sorrelle, N., Zaid, M.M., Toombs, J., Du, W., Wright, S., Hagopian, M., Wang, Z., et al. (2019). Targeting TGFbetaR2-mutant tumors exposes vulnerabilities to stromal TGFbeta blockade in pancreatic cancer. EMBO molecular medicine 11, e10515.

Huang, S., Ernberg, I., and Kauffman, S. (2009). Cancer attractors: a systems view of tumors from a gene network dynamics and developmental perspective. Seminars in cell \& developmental biology 20, 869-876.

lacobuzio-Donahue, C.A. (2012). Genetic evolution of pancreatic cancer: lessons learnt from the pancreatic cancer genome sequencing project. Gut 61, 1085-1094.

Iguchi, E., Safgren, S. L., Marks, D. L., Olson, R. L., and Fernandez-Zapico, M. E. (2016). Pancreatic Cancer, A Mis-interpreter of the Epigenetic Language. The Yale journal of biology and medicine $89,575-590$.

Ikemoto, S., Sugimura, K., Yoshida, N., Wada, S., Yamamoto, K., and Kishimoto, T. (2000). TNF alpha, IL-1 beta and IL-6 production by peripheral blood monocytes in patients with renal cell carcinoma. Anticancer research 20,317-321.

Ivaska, J., Pallari, H.M., Nevo, J., and Eriksson, J.E. (2007). Novel functions of vimentin in cell adhesion, migration, and signaling. Experimental cell research 313, 2050-2062.

Jackson, J.M. (2007). TNF- alpha inhibitors. Dermatologic therapy 20, 251-264. 
Jiao, X., Katiyar, S., Willmarth, N.E., Liu, M., Ma, X., Flomenberg, N., Lisanti, M.P., and Pestell, R.G. (2010). C-Jun induces mammary epithelial cellular invasion and breast cancer stem cell expansion. The Journal of biological chemistry 285, 8218-8226.

Kalbasi, A., Komar, C., Tooker, G.M., Liu, M., Lee, J.W., Gladney, W.L., Ben-Josef, E., and Beatty, G.L. (2017). Tumor-Derived CCL2 Mediates Resistance to Radiotherapy in Pancreatic Ductal Adenocarcinoma. Clinical cancer research : an official journal of the American Association for Cancer Research 23, 137-148.

Kanda, M., Matthaei, H., Wu, J., Hong, S.M., Yu, J., Borges, M., Hruban, R.H., Maitra, A., Kinzler, K., Vogelstein, B., et al. (2012). Presence of somatic mutations in most earlystage pancreatic intraepithelial neoplasia. Gastroenterology 142, 730-733 e739.

Karakhanova, S., Link, J., Heinrich, M., Shevchenko, I., Yang, Y., Hassenpflug, M., Bunge, H., von Ahn, K., Brecht, R., Mathes, A., et al. (2015). Characterization of myeloid leukocytes and soluble mediators in pancreatic cancer: importance of myeloid-derived suppressor cells. Oncoimmunology 4, e998519.

Khorana, A.A., Mangu, P.B., Berlin, J., Engebretson, A., Hong, T.S., Maitra, A., Mohile, S.G., Mumber, M., Schulick, R., Shapiro, M., et al. (2017). Potentially Curable Pancreatic Cancer: American Society of Clinical Oncology Clinical Practice Guideline Update. Journal of clinical oncology : official journal of the American Society of Clinical Oncology 35, 2324-2328.

Kleeff, J., Korc, M., Apte, M., La Vecchia, C., Johnson, C. D., Biankin, A. V., Neale, R. E., Tempero, M., Tuveson, D. A., Hruban, R. H., and Neoptolemos, J. P. (2016). Pancreatic cancer. Nature reviews Disease primers 2, 16022.

Komohara, Y., Jinushi, M., and Takeya, M. (2014). Clinical significance of macrophage heterogeneity in human malignant tumors. Cancer science 105, 1-8.

Koorstra, J.B., Hong, S.M., Shi, C., Meeker, A.K., Ryu, J.K., Offerhaus, G.J., Goggins, M.G., Hruban, R.H., and Maitra, A. (2009). Widespread activation of the DNA damage response in human pancreatic intraepithelial neoplasia. Modern pathology : an official journal of the United States and Canadian Academy of Pathology, Inc 22, 1439-1445.

Krebs, A.M., Mitschke, J., Lasierra Losada, M., Schmalhofer, O., Boerries, M., Busch, H., Boettcher, M., Mougiakakos, D., Reichardt, W., Bronsert, P., et al. (2017). The EMTactivator Zeb1 is a key factor for cell plasticity and promotes metastasis in pancreatic cancer. Nature cell biology 19, 518-529.

Kulbe, H., Thompson, R., Wilson, J.L., Robinson, S., Hagemann, T., Fatah, R., Gould, D., Ayhan, A., and Balkwill, F. (2007). The inflammatory cytokine tumor necrosis factor-alpha generates an autocrine tumor-promoting network in epithelial ovarian cancer cells. Cancer research 67, 585-592. 
Kuleshov, M.V., Jones, M.R., Rouillard, A.D., Fernandez, N.F., Duan, Q., Wang, Z., Koplev, S., Jenkins, S.L., Jagodnik, K.M., Lachmann, A., et al. (2016). Enrichr: a comprehensive gene set enrichment analysis web server 2016 update. Nucleic acids research 44, W90-97.

Larkin, J., Chiarion-Sileni, V., Gonzalez, R., Grob, J.J., Cowey, C.L., Lao, C.D., Schadendorf, D., Dummer, R., Smylie, M., Rutkowski, P., et al. (2015). Combined Nivolumab and Ipilimumab or Monotherapy in Untreated Melanoma. The New England journal of medicine $373,23-34$.

Li, H., and Durbin, R. (2010). Fast and accurate long-read alignment with BurrowsWheeler transform. Bioinformatics 26, 589-595.

Li, J., Byrne, K.T., Yan, F., Yamazoe, T., Chen, Z., Baslan, T., Richman, L.P., Lin, J.H., Sun, Y.H., Rech, A.J., et al. (2018). Tumor Cell-Intrinsic Factors Underlie Heterogeneity of Immune Cell Infiltration and Response to Immunotherapy. Immunity 49, 178-193 e177.

Li, L., Liu, Y.D., Zhan, Y.T., Zhu, Y.H., Li, Y., Xie, D., and Guan, X.Y. (2018). High levels of CCL2 or CCL4 in the tumor microenvironment predict unfavorable survival in lung adenocarcinoma. Thoracic cancer 9, 775-784.

Lind, M.H., Rozell, B., Wallin, R.P., van Hogerlinden, M., Ljunggren, H.G., Toftgard, R., and Sur, I. (2004). Tumor necrosis factor receptor 1 -mediated signaling is required for skin cancer development induced by NF-kappaB inhibition. Proceedings of the National Academy of Sciences of the United States of America 101, 4972-4977.

Lipner, M.B., Peng, X.L., Jin, C., Xu, Y., Gao, Y., East, M.P., Rashid, N.U., Moffitt, R.A., Herrera Loeza, S.G., Morrison, A.B., et al. (2020). Irreversible JNK1-JUN inhibition by JNK-IN-8 sensitizes pancreatic cancer to 5-FU/FOLFOX chemotherapy. JCl insight 5 .

Liou, G.Y., Doppler, H., Necela, B., Krishna, M., Crawford, H.C., Raimondo, M., and Storz, P. (2013). Macrophage-secreted cytokines drive pancreatic acinar-to-ductal metaplasia through NF-kappaB and MMPs. The Journal of cell biology 202, 563-577.

Liu, F., Ai, F., Tian, L., Liu, S., Zhao, L., and Wang, X. (2016). Infliximab enhances the therapeutic effects of 5 -fluorouracil resulting in tumor regression in colon cancer. OncoTargets and therapy 9, 5999-6008.

Logsdon, C.D., and Ji, B. (2009). Ras activity in acinar cells links chronic pancreatitis and pancreatic cancer. Clinical gastroenterology and hepatology : the official clinical practice journal of the American Gastroenterological Association 7, S40-43.

Lopez-Bergami, P., Lau, E., and Ronai, Z. (2010). Emerging roles of ATF2 and the dynamic AP1 network in cancer. Nature reviews. Cancer 10, 65-76. 
Love, M.I., Huber, W., and Anders, S. (2014). Moderated estimation of fold change and dispersion for RNA-seq data with DESeq2. Genome biology 15, 550.

Mantovani, A., Allavena, P., Sica, A., and Balkwill, F. (2008). Cancer-related inflammation. Nature 454, 436-444.

Maurer, C., Holmstrom, S.R., He, J., Laise, P., Su, T., Ahmed, A., Hibshoosh, H., Chabot, J.A., Oberstein, P.E., Sepulveda, A.R., et al. (2019). Experimental microdissection enables functional harmonisation of pancreatic cancer subtypes. Gut 68, 1034-1043.

Mariani, O., Brennetot, C., Coindre, J.M., Gruel, N., Ganem, C., Delattre, O., Stern, M.H., and Aurias, A. (2007). JUN oncogene amplification and overexpression block adipocytic differentiation in highly aggressive sarcomas. Cancer cell 11, 361-374.

Martinelli, P., Carrillo-de Santa Pau, E., Cox, T., Sainz, B., Jr., Dusetti, N., Greenhalf, W., Rinaldi, L., Costello, E., Ghaneh, P., Malats, N., et al. (2017). GATA6 regulates EMT and tumour dissemination, and is a marker of response to adjuvant chemotherapy in pancreatic cancer. Gut 66, 1665-1676.

Mazur, P.K., Herner, A., Mello, S.S., Wirth, M., Hausmann, S., Sanchez-Rivera, F.J., Lofgren, S.M., Kuschma, T., Hahn, S.A., Vangala, D., et al. (2015). Combined inhibition of BET family proteins and histone deacetylases as a potential epigenetics-based therapy for pancreatic ductal adenocarcinoma. Nature medicine 21, 1163-1171.

McWilliams, R. R., Wieben, E. D., Rabe, K. G., Pedersen, K. S., Wu, Y., Sicotte, H., and Petersen, G. M. (2011). Prevalence of CDKN2A mutations in pancreatic cancer patients: implications for genetic counseling. European journal of human genetics : EJHG 19, 472478.

Milan, M., Balestrieri, C., Alfarano, G., Polletti, S., Prosperini, E., Spaggiari, P., Zerbi, A., Diaferia, G.R., and Natoli, G. (2019). FOXA2 controls the cis-regulatory networks of pancreatic cancer cells in a differentiation grade-specific manner. The EMBO journal 38, e102161.

Mini, E., Nobili, S., Caciagli, B., Landini, I., and Mazzei, T. (2006). Cellular pharmacology of gemcitabine. Annals of oncology : official journal of the European Society for Medical Oncology 17 Supp/ 5, v7-12.

Mishra, V.K., Wegwitz, F., Kosinsky, R.L., Sen, M., Baumgartner, R., Wulff, T., Siveke, J.T., Schildhaus, H.U., Najafova, Z., Kari, V., et al. (2017). Histone deacetylase class-I inhibition promotes epithelial gene expression in pancreatic cancer cells in a BRD4- and MYC-dependent manner. Nucleic acids research 45, 6334-6349.

Miyabayashi, K., Baker, L.A., Deschenes, A., Traub, B., Caligiuri, G., Plenker, D., Alagesan, B., Belleau, P., Li, S., Kendall, J., et al. (2020). Intraductal Transplantation 
Models of Human Pancreatic Ductal Adenocarcinoma Reveal Progressive Transition of Molecular Subtypes. Cancer discovery 10, 1566-1589.

Moffitt, R.A., Marayati, R., Flate, E.L., Volmar, K.E., Loeza, S.G., Hoadley, K.A., Rashid, N.U., Williams, L.A., Eaton, S.C., Chung, A.H., et al. (2015). Virtual microdissection identifies distinct tumor- and stroma-specific subtypes of pancreatic ductal adenocarcinoma. Nature genetics 47, 1168-1178.

Motzer, R.J., Escudier, B., McDermott, D.F., George, S., Hammers, H.J., Srinivas, S., Tykodi, S.S., Sosman, J.A., Procopio, G., Plimack, E.R., et al. (2015). Nivolumab versus Everolimus in Advanced Renal-Cell Carcinoma. The New England journal of medicine 373, 1803-1813.

Muckenhuber, A., Berger, A. K., Schlitter, A. M., Steiger, K., Konukiewitz, B., Trumpp, A., Eils, R., Werner, J., Friess, H., Esposito, I., et al. (2018). Pancreatic Ductal Adenocarcinoma Subtyping Using the Biomarkers Hepatocyte Nuclear Factor-1A and Cytokeratin-81 Correlates with Outcome and Treatment Response. Clinical cancer research : an official journal of the American Association for Cancer Research 24, 351359.

Nagarsheth, N., Wicha, M.S., and Zou, W. (2017). Chemokines in the cancer microenvironment and their relevance in cancer immunotherapy. Nature reviews. Immunology 17, 559-572.

Nagrial, A.M., Chin, V.T., Sjoquist, K.M., Pajic, M., Horvath, L.G., Biankin, A.V., and Yip, D. (2015). Second-line treatment in inoperable pancreatic adenocarcinoma: A systematic review and synthesis of all clinical trials. Critical reviews in oncology/hematology 96, 483497.

Natalwala, A., Spychal, R., and Tselepis, C. (2008). Epithelial-mesenchymal transition mediated tumourigenesis in the gastrointestinal tract. World journal of gastroenterology 14, 3792-3797.

Neoptolemos, J.P., Kleeff, J., Michl, P., Costello, E., Greenhalf, W., and Palmer, D.H. (2018). Therapeutic developments in pancreatic cancer: current and future perspectives. Nature reviews. Gastroenterology \& hepatology 15, 333-348.

Neoptolemos, J.P., Palmer, D.H., Ghaneh, P., Psarelli, E.E., Valle, J.W., Halloran, C.M., Faluyi, O., O'Reilly, D.A., Cunningham, D., Wadsley, J., et al. (2017). Comparison of adjuvant gemcitabine and capecitabine with gemcitabine monotherapy in patients with resected pancreatic cancer (ESPAC-4): a multicentre, open-label, randomised, phase 3 trial. Lancet 389, 1011-1024. 
Neph, S., Vierstra, J., Stergachis, A. B., Reynolds, A. P., Haugen, E., Vernot, B., Thurman, R. E., John, S., Sandstrom, R., Johnson, A. K., et al. (2012). An expansive human regulatory lexicon encoded in transcription factor footprints. Nature 489, 83-90.

Neesse, A., Michl, P., Frese, K.K., Feig, C., Cook, N., Jacobetz, M.A., Lolkema, M.P., Buchholz, M., Olive, K.P., Gress, T.M., et al. (2011). Stromal biology and therapy in pancreatic cancer. Gut 60, 861-868.

Nitsche, U., Wenzel, P., Siveke, J.T., Braren, R., Holzapfel, K., Schlitter, A.M., Stoss, C., Kong, B., Esposito, I., Erkan, M., et al. (2015). Resectability After First-Line FOLFIRINOX in Initially Unresectable Locally Advanced Pancreatic Cancer: A Single-Center Experience. Annals of surgical oncology 22 Supp/ 3, S1212-1220.

Noll, E.M., Eisen, C., Stenzinger, A., Espinet, E., Muckenhuber, A., Klein, C., Vogel, V., Klaus, B., Nadler, W., Rosli, C., et al. (2016). CYP3A5 mediates basal and acquired therapy resistance in different subtypes of pancreatic ductal adenocarcinoma. Nature medicine 22, 278-287.

Notta, F., Chan-Seng-Yue, M., Lemire, M., Li, Y., Wilson, G.W., Connor, A.A., Denroche, R.E., Liang, S.B., Brown, A.M., Kim, J.C., et al. (2016). A renewed model of pancreatic cancer evolution based on genomic rearrangement patterns. Nature 538, 378-382.

Olive, K. P., and Tuveson, D. A. (2006). The use of targeted mouse models for preclinical testing of novel cancer therapeutics. Clinical cancer research : an official journal of the American Association for Cancer Research 12, 5277-5287.

Onoue, M., Terada, T., Okuda, M., Fujimoto, K., Doi, R., Imamura, M., and Inui, K. (2004). Surgical resection deteriorates gemcitabine-induced leukopenia in pancreatic cancer. International journal of clinical oncology 9, 174-178.

Patzak, M.S., Kari, V., Patil, S., Hamdan, F.H., Goetze, R.G., Brunner, M., Gaedcke, J., Kitz, J., Jodrell, D.I., Richards, F.M., et al. (2019). Cytosolic 5'-nucleotidase 1A is overexpressed in pancreatic cancer and mediates gemcitabine resistance by reducing intracellular gemcitabine metabolites. EBioMedicine 40, 394-405.

Petrelli, F., Coinu, A., Borgonovo, K., Cabiddu, M., Ghilardi, M., Lonati, V., Aitini, E., Barni, S., and Gruppo Italiano per lo Studio dei Carcinomi dell'Apparato, D. (2015). FOLFIRINOX-based neoadjuvant therapy in borderline resectable or unresectable pancreatic cancer: a meta-analytical review of published studies. Pancreas 44, 515-521.

Puleo, F., Nicolle, R., Blum, Y., Cros, J., Marisa, L., Demetter, P., Quertinmont, E., Svrcek, M., Elarouci, N., lovanna, J., et al. (2018). Stratification of Pancreatic Ductal Adenocarcinomas Based on Tumor and Microenvironment Features. Gastroenterology 155, 1999-2013 e1993. 
Prinz, C. (2012). Pankreas. In Basiswissen Innere Medizin. Springer-Lehrbuch. Heidelberg: Springer Medizin Verlag. pp. 232-246.

Raghavan, S., Winter, P.S., Navia, A.W., Williams,H.L., DenAdel, A., Kalekar, R.L., Galvez-Reyes, J. et al. (2021) "The tumor microenvironment drives transcriptional phenotypes and their plasticity in metastatic pancreatic cancer." BioRxiv, 2020-08.

Qian, B.Z., Li, J., Zhang, H., Kitamura, T., Zhang, J., Campion, L.R., Kaiser, E.A., Snyder, L.A., and Pollard, J.W. (2011). CCL2 recruits inflammatory monocytes to facilitate breasttumour metastasis. Nature 475, 222-225.

Qiao, Y., He, H., Jonsson, P., Sinha, I., Zhao, C., and Dahlman-Wright, K. (2016). AP-1 is a key regulator of proinflammatory cytokine TNFalpha-mediated triple-negative breast cancer progression. The Journal of biological chemistry 291, 18309.

Rahib, L., Smith, B.D., Aizenberg, R., Rosenzweig, A.B., Fleshman, J.M., and Matrisian, L.M. (2014). Projecting cancer incidence and deaths to 2030: the unexpected burden of thyroid, liver, and pancreas cancers in the United States. Cancer research 74, 2913-2921.

Rhim, A.D., Mirek, E.T., Aiello, N.M., Maitra, A., Bailey, J.M., McAllister, F., Reichert, M., Beatty, G.L., Rustgi, A.K., Vonderheide, R.H., et al. (2012). EMT and dissemination precede pancreatic tumor formation. Cell 148, 349-361.

Roe, J.S., Hwang, C.I., Somerville, T.D.D., Milazzo, J.P., Lee, E.J., Da Silva, B., Maiorino, L., Tiriac, H., Young, C.M., Miyabayashi, K., et al. (2017). Enhancer Reprogramming Promotes Pancreatic Cancer Metastasis. Cell 170, 875-888 e820.

Rooman, I., and Real, F.X. (2012). Pancreatic ductal adenocarcinoma and acinar cells: a matter of differentiation and development? Gut 61, 449-458.

Rudin, C.M., Poirier, J.T., Byers, L.A., Dive, C., Dowlati, A., George, J., Heymach, J.V., Johnson, J.E., Lehman, J.M., MacPherson, D., et al. (2019). Molecular subtypes of small cell lung cancer: a synthesis of human and mouse model data. Nature reviews. Cancer 19, 289-297.

Schindelin, J., Arganda-Carreras, I., Frise, E., Kaynig, V., Longair, M., Pietzsch, T., Preibisch, S., Rueden, C., Saalfeld, S., Schmid, B., et al. (2012). Fiji: an open-source platform for biological-image analysis. Nature methods 9, 676-682.

Sehgal, A., Donaldson, D.S., Pridans, C., Sauter, K.A., Hume, D.A., and Mabbott, N.A. (2018). The role of CSF1R-dependent macrophages in control of the intestinal stem-cell niche. Nature communications 9, 1272.

Sethi, G., Sung, B., and Aggarwal, B.B. (2008). TNF: a master switch for inflammation to cancer. Frontiers in bioscience : a journal and virtual library 13, 5094-5107. 
Shadhu, K., and Xi, C. (2019). Inflammation and pancreatic cancer: An updated review. Saudi journal of gastroenterology : official journal of the Saudi Gastroenterology Association 25, 3-13.

Shang, G.S., Liu, L., and Qin, Y.W. (2017). IL-6 and TNF-alpha promote metastasis of lung cancer by inducing epithelial-mesenchymal transition. Oncology letters 13, 46574660 .

Shaulian, E., and Karin, M. (2002). AP-1 as a regulator of cell life and death. Nature cell biology 4, E131-136.

Shen, W., Tao, G.Q., Zhang, Y., Cai, B., Sun, J., and Tian, Z.Q. (2017). TGF-beta in pancreatic cancer initiation and progression: two sides of the same coin. Cell \& bioscience 7, 39 .

Shu, S., Wu, H.J., Ge, J.Y., Zeid, R., Harris, I.S., Jovanovic, B., Murphy, K., Wang, B., Qiu, X., Endress, J.E., et al. (2020). Synthetic Lethal and Resistance Interactions with BET Bromodomain Inhibitors in Triple-Negative Breast Cancer. Molecular cell 78, 10961113 e1098.

Siegel, R.L., Miller, K.D., and Jemal, A. (2020). Cancer statistics, 2020. CA: a cancer journal for clinicians $70,7-30$.

Singh, S. K., Chen, N. M., Hessmann, E., Siveke, J., Lahmann, M., Singh, G., Voelker, N., Vogt, S., Esposito, I., Schmidt, A., et al. (2015). Antithetical NFATc1-Sox2 and p53miR200 signaling networks govern pancreatic cancer cell plasticity. The EMBO journal 34, 517-530.

Singh, S., Hasselluhn, M.C. \& Neesse, A. (2019). A tangled tale of molecular subtypes in pancreatic cancer. Gut 68, 953-954.

Smith, L.M., Wise, S.C., Hendricks, D.T., Sabichi, A.L., Bos, T., Reddy, P., Brown, P.H., and Birrer, M.J. (1999). cJun overexpression in MCF-7 breast cancer cells produces a tumorigenic, invasive and hormone resistant phenotype. Oncogene 18, 6063-6070.

Somerville, T.D.D., Xu, Y., Miyabayashi, K., Tiriac, H., Cleary, C.R., Maia-Silva, D., Milazzo, J.P., Tuveson, D.A., and Vakoc, C.R. (2018). TP63-Mediated Enhancer Reprogramming Drives the Squamous Subtype of Pancreatic Ductal Adenocarcinoma. Cell reports 25, 1741-1755 e1747.

Spaderna, S., Schmalhofer, O., Hlubek, F., Berx, G., Eger, A., Merkel, S., Jung, A., Kirchner, T., and Brabletz, T. (2006). A transient, EMT-linked loss of basement membranes indicates metastasis and poor survival in colorectal cancer. Gastroenterology $131,830-840$. 
Stanger, B.Z., and Hebrok, M. (2013). Control of cell identity in pancreas development and regeneration. Gastroenterology 144, 1170-1179.

Strobel, O., Neoptolemos, J., Jager, D., and Buchler, M.W. (2019). Optimizing the outcomes of pancreatic cancer surgery. Nature reviews. Clinical oncology 16, 11-26.

Suganuma, M., Okabe, S., Marino, M.W., Sakai, A., Sueoka, E., and Fujiki, H. (1999). Essential role of tumor necrosis factor alpha (TNF-alpha) in tumor promotion as revealed by TNF-alpha-deficient mice. Cancer research 59, 4516-4518.

Thalayasingam, N., and Isaacs, J.D. (2011). Anti-TNF therapy. Best practice \& research. Clinical rheumatology 25, 549-567.

Topalian, S.L., Sznol, M., McDermott, D.F., Kluger, H.M., Carvajal, R.D., Sharfman, W.H., Brahmer, J.R., Lawrence, D.P., Atkins, M.B., Powderly, J.D., et al. (2014). Survival, durable tumor remission, and long-term safety in patients with advanced melanoma receiving nivolumab. Journal of clinical oncology : official journal of the American Society of Clinical Oncology 32, 1020-1030.

Tsao, M.S., Marguet, S., Le Teuff, G., Lantuejoul, S., Shepherd, F.A., Seymour, L., Kratzke, R., Graziano, S.L., Popper, H.H., Rosell, R., et al. (2015). Subtype Classification of Lung Adenocarcinoma Predicts Benefit From Adjuvant Chemotherapy in Patients Undergoing Complete Resection. Journal of clinical oncology : official journal of the American Society of Clinical Oncology 33, 3439-3446.

Ueno, T., Toi, M., Saji, H., Muta, M., Bando, H., Kuroi, K., Koike, M., Inadera, H., and Matsushima, K. (2000). Significance of macrophage chemoattractant protein-1 in macrophage recruitment, angiogenesis, and survival in human breast cancer. Clinical cancer research : an official journal of the American Association for Cancer Research 6, 3282-3289.

Vaccaro, V., Sperduti, I., and Milella, M. (2011). FOLFIRINOX versus gemcitabine for metastatic pancreatic cancer. The New England journal of medicine 365, 768-769; author reply 769 .

van Roy, F., and Berx, G. (2008). The cell-cell adhesion molecule E-cadherin. Cellular and molecular life sciences: CMLS 65, 3756-3788.

Vigil, D., Cherfils, J., Rossman, K. L., and Der, C. J. (2010). Ras superfamily GEFs and GAPs: validated and tractable targets for cancer therapy? Nature reviews Cancer 10, 842-857.

Voulgari, A., and Pintzas, A. (2009). Epithelial-mesenchymal transition in cancer metastasis: mechanisms, markers and strategies to overcome drug resistance in the clinic. Biochimica et biophysica acta 1796, 75-90. 
Wang-Gillam, A., Li, C.P., Bodoky, G., Dean, A., Shan, Y.S., Jameson, G., Macarulla, T., Lee, K.H., Cunningham, D., Blanc, J.F., et al. (2016). Nanoliposomal irinotecan with fluorouracil and folinic acid in metastatic pancreatic cancer after previous gemcitabinebased therapy (NAPOLI-1): a global, randomised, open-label, phase 3 trial. Lancet 387, 545-557.

Wajant, H. (2009). The role of TNF in cancer. Results and problems in cell differentiation 49, 1-15.

Waters, A. M., and Der, C. J. (2018). KRAS: The Critical Driver and Therapeutic Target for Pancreatic Cancer. Cold Spring Harbor perspectives in medicine 8.

Wellner, U., Schubert, J., Burk, U.C., Schmalhofer, O., Zhu, F., Sonntag, A., Waldvogel, B., Vannier, C., Darling, D., zur Hausen, A., et al. (2009). The EMT-activator ZEB1 promotes tumorigenicity by repressing stemness-inhibiting microRNAs. Nature cell biology 11, 1487-1495.

Wilentz, R.E., Geradts, J., Maynard, R., Offerhaus, G.J., Kang, M., Goggins, M., Yeo, C.J., Kern, S.E., and Hruban, R.H. (1998). Inactivation of the p16 (INK4A) tumorsuppressor gene in pancreatic duct lesions: loss of intranuclear expression. Cancer research 58, 4740-4744.

Wu, S.Y., Lee, A.Y., Lai, H.T., Zhang, H., and Chiang, C.M. (2013). Phospho switch triggers Brd4 chromatin binding and activator recruitment for gene-specific targeting. Molecular cell 49, 843-857.

Xia, X., Wu, W., Huang, C., Cen, G., Jiang, T., Cao, J., Huang, K., and Qiu, Z. (2015). SMAD4 and its role in pancreatic cancer. Tumour biology : the journal of the International Society for Oncodevelopmental Biology and Medicine 36, 111-119.

Yadav, D., and Lowenfels, A.B. (2013). The epidemiology of pancreatitis and pancreatic cancer. Gastroenterology 144, 1252-1261.

Ying, H., Dey, P., Yao, W., Kimmelman, A.C., Draetta, G.F., Maitra, A., and DePinho, R.A. (2016). Genetics and biology of pancreatic ductal adenocarcinoma. Genes \& development 30, 355-385.

Zhang, J., Lu, Y., and Pienta, K.J. (2010). Multiple roles of chemokine (C-C motif) ligand 2 in promoting prostate cancer growth. Journal of the National Cancer Institute 102, 522528.

Zhang, Q.W., Liu, L., Gong, C.Y., Shi, H.S., Zeng, Y.H., Wang, X.Z., Zhao, Y.W., and Wei, Y.Q. (2012). Prognostic significance of tumor-associated macrophages in solid tumor: a meta-analysis of the literature. PloS one 7, e50946. 
Zhang, Z., Lin, G., Yan, Y., Li, X., Hu, Y., Wang, J., Yin, B., Wu, Y., Li, Z., and Yang, X.P. (2018). Transmembrane TNF-alpha promotes chemoresistance in breast cancer cells. Oncogene 37, 3456-3470.

Zhao, X., Fan, W., Xu, Z., Chen, H., He, Y., Yang, G., Yang, G., Hu, H., Tang, S., Wang, P., et al. (2016). Inhibiting tumor necrosis factor-alpha diminishes desmoplasia and inflammation to overcome chemoresistance in pancreatic ductal adenocarcinoma. Oncotarget 7, 81110-81122.

Zhao, R., Choi, B. Y., Lee, M. H., Bode, A. M., and Dong, Z. (2016). Implications of Genetic and Epigenetic Alterations of CDKN2A (p16(INK4a)) in Cancer. EBioMedicine 8, 30-39.

Zhou, Q., and Melton, D.A. (2018). Pancreas regeneration. Nature 557, 351-358.

Zidi, I., Mestiri, S., Bartegi, A., and Amor, N.B. (2010). TNF-alpha and its inhibitors in cancer. Medical oncology 27, 185-198.

Zoetemelk, M., Ramzy, G.M., Rausch, M., and Nowak-Sliwinska, P. (2020). Drug-Drug Interactions of Irinotecan, 5-Fluorouracil, Folinic Acid and Oxaliplatin and Its Activity in Colorectal Carcinoma Treatment. Molecules 25. 


\section{Acknowledgements}

"Everything happens for the best". In retrospect, I am very glad that I have made the decision to take the doctoral adventure which is particularly worthy and memorable. The last four years have been integrated with both highlighted moments of achievement and adverse circumstances. When looking back, it is like an evolution - I have been equipped with higher learning capacity and passion, as well as more strength to handle failure and dark time in life. I owe all those to the people who accompanied me throughout the whole Ph.D. journey. What I achieved today will never ever be possible without you.

First of all, I sincerely thank my supervisor Dr. Shiv K. Singh for guiding me with this super interesting research project. It has sure been a wonderful time working with him. He has given me continuous guidance and encouragement with his great patience throughout my doctoral study. I really admire his delicate perception of the front edge research and newly developed methods which always inspires the whole working group. He is a great mentor of leading me how to think logically and scientifically. It was really a great honor to be a member of this active and excellent lab.

On a special note, I am very grateful to Prof. Dr. med. Volker Ellenrieder for providing me the opportunity to work in this collaborative department. I really appreciate Prof. Dr. Sabine Mihm for helping me settle in Göttingen. I thank PD. Dr. med. Elisabeth Hessmann for all the support and help during my Ph.D.

I would like to express sincere gratitude to my thesis advisory committee members Prof. Dr. Holger Reichardt and Prof. Dr. Dieter Kube for their constructive advice and feedback of my project during the TAC meetings throughout my Ph.D. years. Moreover, I would like to thank Dr. Ufuk Günesdogan and Prof. Dr. Argyris Papantonis for the highly efficient cooperations and the immense help in the NGS data processing. Besides, I am very thankful to my external examination members Prof. Dr. Argyris Papantonis, Dr. Ufuk Günesdogan, and Dr. Nico Posnien for their great support of my thesis defense arrangement. 
Additionally, I would like to thank the Chinese Scholarship Council (CSC) for the financial support which enables my study in Germany.

Furthermore, I am grateful to have received plenty of supports from my wonderful lab mates. I specially thank my best Ph.D. mate Lukas Klein - who is the most knowledgeable person I have ever known. He is not only the source of my fun time in the lab, but also a great helper for assisting me in conducting animal experiments and bioinformatics. I would like to thank Laura Urbach for always being available whatever questions I raise; Frederike Penz for her excellent technical support.

Very special thanks to all the people whom I have the honor to collaborate with: Xiaojuan Li from AG Günesdogan for the RNA-seq analysis and Dr. Theodoros Georgomanolis from AG Papantonis for the ATAC-seq and ChIP-seq analysis.

My days in Göttingen would not have been the same without a couple of friends. They have provided me precious company and been staying with me regardless of the situation I encountered in my life. I am extremely lucky to have my friends Shilpa Patil and Zhe Zhang, for all the movie nights, dinner treats, Dewali fun nights and especially the trip to Croatia. It is amazing that our minds are so much in sync, I will remember all those jokes of which only we ourselves know the meaning. I am very thankful to my friends Xin Wang, Yao Wang, Shuwen Shan, Fangzheng Xu, and Shu Zhang who consistently take care of me and try their best to enrich my spare life.

Furthermore, I would like to extend my thanks to all the former and current colleagues of the Department of Gastroenterology, GI Oncology and Endocrinology. I would also like to express my thanks to Ph.D. and Postdoc fellows - Geske Schmidt, Umair Latif, Lennar Versemann, Nina Pfisterer, Maria Ulisse, Anna Weber, Marie Hasselluhn, Melanie Patzak and Iswarya Ramu, for all the helpful advice and encouragement. I would further like to acknowledge the superior technical support from Waltraut Kopp, Kristina Reutlinger, Jessica Spitalieri and Dr. Sercan Mercan. 
Last but not least, I whole-heartedly thank my parents, who are the biggest supporters of my life. My strength and power in pursuing my dreams was attributed to their limitless trust and faith in me. 